\title{
Electronic excitations: density-functional versus many-body Green's-function approaches
}

\author{
Giovanni Onida* \\ Istituto Nazionale per la Fisica della Materia, Dipartimento di Fisica dell' Università di \\ Roma "Tor Vergata," Via della Ricerca Scientifica 1, I-00133 Roma, Italy \\ Lucia Reining ${ }^{\dagger}$ \\ Laboratoire des Solides Irradiés, UMR 7642 CNRS-CEA, École Polytechnique, \\ F-91128 Palaiseau, France \\ Angel Rubio ${ }^{\ddagger}$ \\ Departamento de Física de Materiales, Facultad de Químicas, Universidad del País Vascol \\ Euskal Herriko Unibertsitatea, Centro Mixto CSIC-UPV/EHU and Donostia International \\ Physics Center (DIPC) 20018 San Sebastián/Donostia, Spain \\ and Laboratoire des Solides Irradiés, UMR 7642 CNRS-CEA, École Polytechnique, \\ F-91128 Palaiseau, France
}

(Published 7 June 2002)

\begin{abstract}
Electronic excitations lie at the origin of most of the commonly measured spectra. However, the first-principles computation of excited states requires a larger effort than ground-state calculations, which can be very efficiently carried out within density-functional theory. On the other hand, two theoretical and computational tools have come to prominence for the description of electronic excitations. One of them, many-body perturbation theory, is based on a set of Green's-function equations, starting with a one-electron propagator and considering the electron-hole Green's function for the response. Key ingredients are the electron's self-energy $\Sigma$ and the electron-hole interaction. A good approximation for $\Sigma$ is obtained with Hedin's $G W$ approach, using density-functional theory as a zero-order solution. First-principles $G W$ calculations for real systems have been successfully carried out since the 1980s. Similarly, the electron-hole interaction is well described by the Bethe-Salpeter equation, via a functional derivative of $\Sigma$. An alternative approach to calculating electronic excitations is the time-dependent density-functional theory (TDDFT), which offers the important practical advantage of a dependence on density rather than on multivariable Green's functions. This approach leads to a screening equation similar to the Bethe-Salpeter one, but with a two-point, rather than a four-point, interaction kernel. At present, the simple adiabatic local-density approximation has given promising results for finite systems, but has significant deficiencies in the description of absorption spectra in solids, leading to wrong excitation energies, the absence of bound excitonic states, and appreciable distortions of the spectral line shapes. The search for improved TDDFT potentials and kernels is hence a subject of increasing interest. It can be addressed within the framework of many-body perturbation theory: in fact, both the Green's functions and the TDDFT approaches profit from mutual insight. This review compares the theoretical and practical aspects of the two approaches and their specific numerical implementations, and presents an overview of accomplishments and work in progress.
\end{abstract}

\section{CONTENTS}

I. Introduction

A. Motivation from experiments

602

B. Theoretical framework

1. Overview

603

603

2. Effective Hamiltonians and effective interactions

604

II. TDDFT and Green's-Function Approaches:

Common Ingredients

608

*Present address: Istituto Nazionale per la Fisica della Materia, Dipartimento di Fisica dell' Universitá di Milano, via Celoria 16, I-20133 Milano, Italy. Electronic address: Giovanni.Onida@mi.infn.it

${ }^{\dagger}$ Electronic address: Lucia.Reining@polytechnique.fr

Electronic address: arubio@sc.ehu.es
A. Density-functional theory for the ground state

B. The inverse dielectric function and local-field effects

C. Four-point kernels and effective two-particle equations

III. Green's-Function Theory

A. The concept of Green's functions and the selfenergy

1. Connection to spectroscopic measurements

2. Dyson's equation: the self-energy operator $\Sigma$

B. Hedin's equations

C. The iterative approach

1. First iteration step: the $G W$ approximation

2. Second iteration step: vertex correction in $P$ and $\Sigma$

IV. Green's Functions in Practice

A. Calculations of one-particle excited states 1. $G W$ calculations
621

621 
a. Evaluation of the Green's function $G$

b. Evaluation of the screened Coulomb interaction $W=\varepsilon^{-1} v$

c. Application of the self-energy $\Sigma$

2. Comparison of $G W$ and DFT results

B. Calculations of two-particle excited states

1. Independent-quasiparticle approximation

2. Electron-hole attraction

3. The effects of the electron-hole interaction

4. Different levels of sophistication

V. Time-Dependent Density-Functional Theory

A. Formalism

B. Excitation energies in TDDFT

C. The exchange-correlation kernel $f_{x c}$

VI. TDDFT Versus Bethe-Salpeter
A. The equations
B. The limit of isolated electrons
C. Relaxation and correlation
D. Comparison of some applications
1. Common ingredient: the bare Coulomb interaction
2. Exchange and correlation effects
a. Some applications to finite systems
b. Some applications to extended systems

VII. Conclusions-Frequently Asked Questions and Open Questions

Acknowledgments

Appendix A: Exact Properties of $f_{x c}$

Appendix B: Derivation of the Equations Common to the TDDFT and Bethe-Salpeter Approaches

1. Dyson-like equation for the macroscopic dielectric function

2. Effective two-particle equations

Appendix C: An $f_{x c}$ from the Bethe-Salpeter Approach References

\section{INTRODUCTION}

In every spectroscopic experiment one perturbs the sample (by incoming photons, electrons, etc.) and measures the response of the system to this perturbation. In other words, the system is excited. Therefore it is in general not sufficient to calculate ground-state properties in order to interpret or predict results of experiments like photoemission, electron-energy loss, absorption, etc.

Direct and inverse photoemission and absorption can be taken as the prototype spectroscopies which one would like to describe in this context. They are schematically depicted in Fig. 1. In the photoemission process the system absorbs a photon $h \nu$, and an electron is ejected whose kinetic energy $E_{k}$ is then measured at some distance. If one considers this photoelectron to be completely decoupled from the sample, energy and momentum conservation allow one to deduce the change in total energy of the sample, which is interpreted as the energy level of the "hole," i.e., the level that was formerly occupied by the photoelectron. Hence as a first approach one can state that photoemission measures the density of occupied states. By analogy, inverse photoemission yields information about the density of unoccupied states. In absorption experiments, an electron is excited from an occupied state into a conduction state. This process looks, at first glance, like the sum of a pho- toemission and an inverse photoemission experiment (creation of a hole and an electron). Instead, as shown in Fig. 1, in the absorption measurement the excited electron remains inside the system and cannot be supposed to be a free electron decoupled from the others. Hence whereas direct and inverse photoemission results are often already well described by the density of occupied and unoccupied states, respectively, one realizes that (i) in absorption, the joint density of occupied and unoccupied states must be considered, (ii) even over a small range of excitation energies, transition probabilities can vary considerably and must be taken into account, and (iii) the excited electron and the hole cannot be treated separately, since the electron feels the presence of the hole. Point (iii) constitutes the main difficulty for a correct description of this type of experiment.

The interpretation of photoemission spectra as a density of occupied states is linked to the picture of independent electrons which occupy some well-defined energy level in the system. Of course, electrons are not independent, and it is clear that, for example, an electron that leaves the sample will lead the remaining electrons to relax. This relaxation energy and other quantum-mechanical contributions must be taken into account if energy differences are to be calculated correctly. In other words, in photoemission the singleelectron energy levels are renormalized by the presence of the other electrons. One can then still retain the picture of one-particle energy levels, but these particles are quasielectrons and quasiholes, i.e., they contain the effects of all the other particles (Landau, 1957a, 1957b, 1959). They can still be described by a sort of oneparticle Schrödinger equation, which does, however, contain rather complicated effective potentials (also called optical potentials) reflecting these interactions. Moreover, it is clear that, in the case of absorption, even a very sophisticated one-quasiparticle Schrödinger equation would be inadequate, since the quasielectron and quasihole must be described simultaneously, requiring

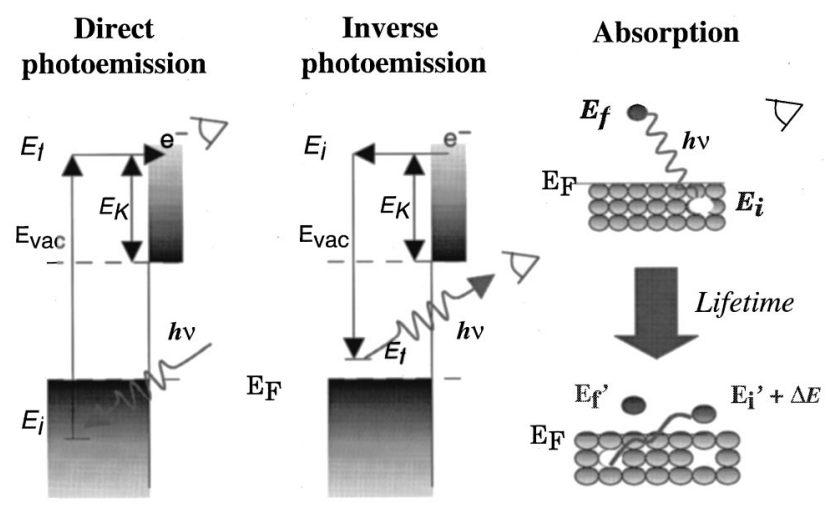

FIG. 1. Schematic representation of the excitations involved in direct photoemission, inverse photoemission, and absorption spectroscopies. Photoemission can be resolved in angle, spin, and time; absorption can be resolved in polarization and time. This allows a direct probing of the electronic and structural properties of bulk and low-dimensional samples including dynamical effects. $\Delta E=E_{f}-E_{f}^{\prime}$, apart from phonons and radiative losses. 
an effective two-particle equation. Progress in the theoretical description of spectroscopy is in fact very often linked to progress in finding better effective one- or twoparticle Hamiltonians.

One important motivation for seeking more precise theoretical descriptions is, of course, the fact that discrepancies arise between experimental spectra and the spectra calculated at some approximate level. This motivation becomes stronger as more precise experiments become available.

\section{A. Motivation from experiments}

The excitations considered in the present work concern electrons, mainly in the valence energy region. Most of the theoretical tools presented in the following are general enough to be used also for the study of excitations involving core electrons, although in that case one is frequently interested in more complex phenomena such as the Auger effect (Verdozzi et al., 2001), which is not explicitly treated in this review. Traditional techniques for the study of valence electron excitations use either photons (visible and ultraviolet absorption, transmission and reflectivity spectra), electrons (electron-energy-loss spectra), or both (electron photoemission and inverse photoemission spectra).

During the last two decades, important improvements in many of these techniques have been achieved, due to the ongoing substantial progress in obtaining (a) high spectral and spatial resolution, brightness; (b) short measurement times (scale of femtoseconds); (c) high spatial coherence; and (d) low temperatures (Smith, 2001). Large contributions come from the progress made at synchrotron-radiation sources and with ultrafast lasers. In particular, photon beams in the soft-x-ray energy region (extreme ultraviolet) are increasingly used, due to the availability of high-brilliance sources (Smith, 2001). Several striking examples can be found in the recent literature. Femtosecond lasers yield information about elementary electronic processes occurring at surfaces on time scales from pico- to femtoseconds, which are relevant for potential technological applications. For example, an electronic excitation is the initial step in many chemical reactions, and the energetics and lifetime of this process directly govern the reaction probability. Hence chemical selectivity can be obtained through an activation of the desired reaction via a femtosecond electronic excitation (Sundström, 1996; see Diau et al., 1998, for an example). Time-resolved femtosecond photoemission spectroscopy has been used to gain insight into electronically induced adsorbate reactions at surfaces and kinetics of growth, and to monitor in real time and with atomic resolution the dynamics of electrons (Plummer, 1997) and the motion of atoms at surfaces (Petek et al., 2000). Recent advances in ultrafast electron diffraction have yielded direct imaging of transient structures in chemical reactions (Ihee et al., 2001). X-ray microscopy allowed the study of biological matter, like the internal structure of a cell, with a spatial resolution of $36 \mathrm{~nm}$ using photons in the "water window" energy range, i.e., between 290 and $530 \mathrm{eV}$, where carbon absorbs (excitation of the $1 s$ electrons) and water is relatively transparent. Photoelectron spectra with high spatial resolution are at the basis of photoelectron microscopy, where a $20-\mathrm{nm}$ resolution can be obtained (Nolting et al., 2000). The determination of the Fermi surface of very complex materials such as cuprate superconductors (Zhou et al., 1999), the study of a Fermi gap opening in complex structures (Cepek et al., 2001), and the measurement of the energy dependence of the linewidth associated with photoemission peaks near the Fermi energy (Valla et al., 1999a, 1999b) are now possible because of the improved spectral resolution.

Often, it is clear that a simple one-particle picture is intrinsically inadequate for describing the processes occurring in the experiments. A good example is resonant photoemission, where core-valence absorption and valence electron Auger emission interfere. Many aspects of these experiments can in principle be described by the theoretical tools discussed in this review. In the following, we define the common framework of the approaches and then consider and compare them in detail.

\section{B. Theoretical framework}

\section{Overview}

This review treats approaches using or leading to the picture of effective particles, i.e., "quasiparticles," as outlined above. There are, of course, other ways to treat the many-body problem, e.g., the configurationinteraction approach of quantum chemistry (see, for example, Jensen, 1999; Szabo and Ostlund, 1983; BonacićKoutecký et al., 1990 for an application). The latter is based on the minimization of the energy with respect to the expansion coefficients of a trial many-body wave function, written as a linear combination of determinants. A description of configuration-interaction-based techniques is clearly beyond the scope of this paper, although they can be very efficient (e.g., in the determination of higher excited states for point defects in $\mathrm{SiO}_{2}$; Raghavachari et al., 2002). However, the drawback of the configuration-interaction method with respect to density-functional-based or Green's-function-based "quasiparticle" methods is its unfavorable scaling with the system's size. ${ }^{1}$

\footnotetext{
${ }^{1}$ In particular, full configuration-interaction calculations involve a number of determinants which grows exponentially with the size of the system. Simplified methods have been devised, such as the truncated configuration interaction, which reduces the scaling to $M^{6}$ (where $M$ is the number of basis functions) when only determinants with a number of excited electrons lower than or equal to 2 are included. Other scalings, namely, $M^{8}$ or $M^{10}$, are obtained by also including triply or quadruply excited determinants. The approaches which will be discussed in this review can exhibit a much better scaling, according to the system and to the numerical implementation (Benedict et al., 1998a; Bertsch et al., 2000; Hahn et al., 2002; Vasiliev et al., 2002).
} 
The concept of quasiparticles is intimately linked to that of band structure (or discrete energy levels in a finite system), and the corresponding one-electron-like Schrödinger equations. Band-structure equations can contain the complications of electron-electron interactions implicitly in empirical or semiempirical Hamiltonians, as in the tight-binding approach, or they can treat those effects explicitly. It is this latter point which is of interest here. We shall hence discuss the quasiparticle formalism of many-body perturbation theory. In addition, we shall also discuss approaches based on the static and time-dependent density-functional theory (DFT and TDDFT).

Static DFT (Hohenberg and Kohn, 1964) in the formulation of Kohn and Sham (1965) is a ground-state theory in the form of an effective one-particle Schrödinger equation (the Kohn-Sham equation). The KohnSham eigenvalues are often interpreted as quasiparticle energies, and their differences as optical excitation energies, without formal justification. The Kohn-Sham eigenvalues and eigenfunctions are also often used as starting point for further excited-state calculations. On the other hand, DFT has been extended to time-dependent DFT (Runge and Gross, 1984), which is in principle an exact theory for the description of neutral excitations (such as those involved in absorption). In fact, TDDFT can be written in the form of an effective two-particle equation, which can be directly compared to the effective twoquasiparticle equation of many-body perturbation theory.

In the last few years, several reviews have addressed different aspects of the calculation of electronic excitations in both finite and infinite systems using either the quasiparticle picture (see, e.g., Aryasetiawan and Gunnarsson, 1998; Strinati 1988; Aulbur et al., 1999; Farid, 1999a; Hedin, 1999; Rohlfing and Louie, 2000) or TDDFT (see, for example, Gross et al., 1994, 1996; Casida 1995, 1996; Dobson, Vignale, and Das, 1997; Rubio et al., 1997; van Leeuwen, 2001; Burke et al., 2002). It emerges that, although both methods are in principle exact when applied to the appropriate problem, they present different drawbacks: in the context of manybody perturbation theory, the effective one- and twoquasiparticle Hamiltonians are essentially well established, but the methods become numerically impracticable for complex systems. TDDFT, on the other hand, could in principle lead to technically simpler equations, but its large-scale application is at present prevented by the fact that a good general approximation for the corresponding effective two-particle Hamiltonian (or equivalent quantities) has not yet been found. The large increase of interest in theoretical spectroscopic analysis from first principles suggests a joint venture in exploring both approaches. The aim is to reach an effective method of handling excitations of many-electron systems with an effort comparable to that of DFT-based approaches used for the calculation of ground-state properties.

In the present work we review some of the essential physics contained in the various approximations used to describe spectroscopic measurements from first principles. We address in detail some frequently asked questions related to the calculation of photoemission and optical spectra, including those which remain open and may be solved in the near future. We attempt to give a general perspective of our present understanding of different spectroscopies and to compare the many-body perturbation theory approaches-in particular those based on Hedin's equations (Hedin, 1965) - with timedependent density-functional theory. This comparison with an eye to practical applications, together with the focus on the calculation of photon absorption and electron-energy-loss spectra which involve twoquasiparticles (electron-hole) excitations, characterizes the present work and differentiates it from other recently published reviews on Green's-function methods, more focused on one-particle Green's functions and single-quasiparticle excitations, such as those involved in photoemission or inverse photoemission (e.g., Aryasetiawan and Gunnarsson, 1998; Aulbur et al., 1999; Farid, 1999a; Hedin, 1999).

Since this field of research is rather broad, and in order to make the discussions more focused and specific, we have restricted the review to the description of purely electronic excitations from valence states, for nonmagnetic systems. We have hence excluded the explicit discussion of core-level spectroscopy (Almbladh and Hedin, 1983) and Auger spectroscopy (Verdozzi et al., 2001), although the theory presented here remains valid in that energy range. We also exclude the problem of strong correlation and the methods that have been developed to treat these problems in particular, e.g., the local-density approximation plus an on-site Hubbard repulsion method (LDA+U; Anisimov et al., 1997) and the dynamical mean-field theory (Georges et al., 1996).

Unless otherwise stated, we shall use atomic units throughout the paper (i.e., $e^{2}=\hbar=m_{e}=1$ ).

\section{Effective Hamiltonians and effective interactions}

The earliest attempts to cast the many-body problem into the form of one particle moving in some mean (or, more generally, effective) field due to the electronelectron interaction are the Hartree (1928) and the Hartree-Fock (Fock, 1930) approaches: a product or determinant ansatz for the many-body wave function allows one to minimize the total energy variationally, leading to an effective one-particle Schrödinger equation. It is interesting to note that the Hartree-Fock method already yields good results, like total energy differences, in certain systems. However, the interpretation of the Hartree-Fock eigenvalues as electron addition and removal energies does not lead to satisfactory agreement between theory and experiment. As a guideline we may take the minimum direct quasiparticle gap at $\Gamma$ in diamond, for which Mauger and Lannoo (1977) obtained a value of $E_{g} \simeq 15 \mathrm{eV}$ in a self-consistent Hartree-Fock calculation, instead of the experimental quasiparticle gap of $7.3 \mathrm{eV}$ (inferred from optical experiments by Roberts and Walker, 1967). 
The need for going beyond Hartree-Fock in electronic structure calculations was in fact recognized early. Many-body correlation effects have been studied since the 1930s (Wigner, 1934); an important milestone is the work of Quinn and Ferrell (1958) on the homogeneous electron gas and metals. A key role is played by the electron self-energy, which is the proper exchangecorrelation potential acting on an excited electron or hole. A sound basis for modern band-structure calculations was laid by Hedin (Hedin, 1965; Hedin and Lundqvist, 1969) with the so-called $G W$ approximation for the self-energy, which was applied to the electron gas in that work. The essential improvement offered by the $G W$ one-particle Schrödinger equation over the Hartree-Fock equation lies in the explicit description of the potential induced by the additional particle. In order to understand this, one might ask why the eigenvalues of the Hartree-Fock equation do not yield a satisfactory description of quantities like the band structure, although Koopman's theorem says that it is justified to identify eigenvalues with total energy differences. So what is missing, and which ingredients should reasonably appear in a theory going beyond Hartree-Fock and allowing one to use the eigenvalues of some effective Hamiltonian?

First, Koopman's theorem supposes that the oneelectron orbitals are frozen upon changing the number of electrons. In order to go beyond this drastic approximation, one has to include the relaxation of the orbitals as a response to the addition of an electron or a hole to the system. This effect is contained in the so-called deltaself-consistent-field calculations for finite systems, where the difference in total energy between two selfconsistent calculations, for $N$ and $N \pm 1$ electrons, is obtained explicitly. In other words, total energy differences are calculated successfully within Hartree-Fock, because in the calculation for $N \pm 1$ electrons the wave functions are allowed to relax with respect to those of the $N$-electron calculation. This response of the system to the additional electron or hole-the screening of the additional particle — can be reformulated in linear response in terms of the dielectric matrix $\varepsilon$, defined by the relation

$$
V_{t o t}(\mathbf{r})=\int d \mathbf{r}^{\prime} \varepsilon^{-1}\left(\mathbf{r}, \mathbf{r}^{\prime}\right) V_{\text {ext }}\left(\mathbf{r}^{\prime}\right)
$$

between the external potential $V_{\text {ext }}$ and the screened (total) potential $V_{\text {tot }}$. The total potential is the sum of the external potential and the potential due to the polarization of the system induced by the external perturbation. It is the relaxation of the wave functions which gives rise to this polarization, and one can therefore try to use the concept of the dielectric matrix in order to correct the Hartree-Fock one-particle Schrödinger equation, without calculating the relaxation explicitly as in the delta-self-consistent-field approach. One has hence to calculate the induced potential $V_{\text {ind }}=V_{t o t}-V_{e x t}$ due to an additional electron, and acting on that electron itself. As a first step, one could assume that the additional electron in a state $n$ gives rise to an induced potential that is proportional to the perturbing "charge density" $\left|\psi_{n}(\mathbf{r})\right|^{2}$ (as in the familiar picture of the image charge). However, whereas Hartree relaxation effects are very important in small systems, in solids in a Bloch picture they are negligible, and hence this effect alone cannot lead to a satisfactory correction of the HartreeFock equation. Rather, one should take $\left|\psi_{n}(\mathbf{r})\right|^{2}$ as the probability to find the additional electron in some point $\mathbf{r}$, and then, supposing that the additional electron is at $\mathbf{r}$ (i.e., taking correlation into account), calculate the induced potential in the same point. Then, in the corresponding equations $\left|\psi_{n}\right|^{2}$ is replaced by a $\delta$ function, and the potential which should be added to the HartreeFock equation turns out to be the so-called Coulombhole term $\frac{1}{2} v\left(\varepsilon^{-1}-1\right), v$ being the bare Coulomb interaction. $^{2}$

Of course, the change in the charge distribution due to the additional, perturbing, electron will also affect the exchange term of the original Hartree-Fock equation, which now turns out to be screened by $\varepsilon^{-1}$. These arguments lead to an effective one-particle Schrödinger equation for an additional electron (or hole) which reads

$$
\begin{aligned}
(- & \frac{\nabla^{2}}{2}+\int d \mathbf{r}^{\prime} \rho\left(\mathbf{r}^{\prime}\right) v\left(\mathbf{r}, \mathbf{r}^{\prime}\right) \\
& \left.+\frac{1}{2} \int d \mathbf{r}^{\prime} v\left(\mathbf{r}^{\prime}, \mathbf{r}\right)\left[\varepsilon^{-1}\left(\mathbf{r}, \mathbf{r}^{\prime}\right)-\delta\left(\mathbf{r}, \mathbf{r}^{\prime}\right)\right]\right) \psi_{n}(\mathbf{r}) \\
& -\int d \mathbf{r}^{\prime} W\left(\mathbf{r}, \mathbf{r}^{\prime}\right) \sum_{s=1}^{o c c} \psi_{s}^{*}\left(\mathbf{r}^{\prime}\right) \psi_{s}(\mathbf{r}) \psi_{n}\left(\mathbf{r}^{\prime}\right)=\epsilon_{n} \psi_{n}(\mathbf{r}),
\end{aligned}
$$

where $W=\varepsilon^{-1} v$ is the screened Coulomb interaction. This is the equation known as the static COHSEX (Coulomb hole plus screened exchange) approximation (Hedin, 1965), where the terms added to the kinetic-energy operator and the Hartree potential constitute the selfenergy $\Sigma$ COHSEX. For diamond, Hybertsen and Louie (1986) have found that the energy difference between valence and conduction bands is overestimated in the COHSEX approximation by about $1 \mathrm{eV}$, which means, however, that most of the error of the Hartree-Fock result cited above is removed by this correction.

To go further, one must take into account the fact that the response of a system to an external perturbation is frequency dependent, which means that one has to introduce the concept of a response in time (dynamical correlation and memory effects). In fact, the COHSEX potential is nothing other than the static limit of the exchange-correlation self-energy calculated in the so-

\footnotetext{
${ }^{2}$ The factor $\frac{1}{2}$ describes the fact that a charge $e$ is taken from infinity to a point $\mathbf{r}$ of the system or, equivalently, built up from $e=0$. The part of the work coming from the induced charge which is needed to do this is $\int_{0}^{e} d q V_{\text {ind }}(q)=\int_{0}^{e} d q q\left(\varepsilon^{-1}-1\right) v$ $=1 / 2 e^{2} *(W-v)$. This is nothing other than the adiabatic building up of charge density (Hedin, 1965, 1999) of classical electrostatics.
} 
called $G W$ approximation (Hedin, 1965), which, as pointed out above, can be considered as the state-of-theart tool for band-structure calculations today.

After its introduction for metals, the $G W$ approximation was also used early for semiconductors and insulators, first in static COHSEX (Brinkman and Goodman, 1966; Lipari and Fowler, 1970; Brener, 1975a, 1975b), later using a plasmon-pole approximation in a model $\varepsilon^{-1}$ (Bennett and Inkson, 1977; Inkson and Bennett, 1978) and, starting from a Hartree-Fock calculation, using a realistic frequency- and wave-vector-dependent dielectric matrix, in a basis of localized orbitals (Strinati et al., 1980, 1982).

It was shown that the $G W$ approach managed to correct the largest part of the band-gap error; in fact, for the example of the gap at $\Gamma$ in diamond, Strinati et al. (1980) found a value of $7.4 \mathrm{eV}$, which is half of the Hartree-Fock gap and very close to the experimental one. ${ }^{3}$ The fact that the inclusion of dynamical effects in the response functions leads to a reduction of the quasiparticle gap with respect to the static COHSEX result is a general finding, and the example of diamond shows the typical order of magnitude of the dynamical effects (i.e., about $10-20 \%$ of the experimental gap).

On the other hand, $G W$ calculations are cumbersome as compared, for example, to DFT ones. As pointed out above, the Kohn-Sham equation also has the form of an effective one-particle Schrödinger equation, and it is therefore tempting to use its eigenvalues as electron addition and removal energies. However, this yields large errors (Lundqvist and March, 1983); in particular one finds a strong underestimate of the band gap of semiconductors and insulators. For diamond, the difference of Kohn-Sham eigenvalues calculated in the local-density approximation (LDA) has yielded a gap at $\Gamma$ of $E_{g}$ $=5.51 \mathrm{eV}$ (Hybertsen and Louie, 1985). The origin of this failure, whether it is mainly due to the wrong interpretation of the Kohn-Sham equation or to the LDA, is still discussed today and will be studied in Sec. IV.A.2. In any case, DFT is a good starting point for further calculations, and starting from the work of Hybertsen and Louie $(1985,1986)$, and Godby et al. (1986, 1987, 1988), today ab initio $G W$ calculations are mostly performed using Kohn-Sham results as ingredients (to be precise, the Kohn-Sham eigenvalues and eigenfunctions are used to construct the self-energy of the $G W$ form). These fully ab initio $G W$ calculations generally yield very good agreement, most often better than $10-15 \%$, between the experimental and the calculated band structure, apart from the case of strongly correlated systems. Again for the example of diamond, Hybertsen and Louie (1985) and Godby et al. (1987) have performed $a b$ initio $G W$ calculations and obtained a quasiparticle gap at $\Gamma$ of $E_{g}=7.38 \mathrm{eV}$ and $E_{g}=7.26 \mathrm{eV}$, respectively, which compare well with the experimental value of 7.3 eV (Roberts and Walker, 1967).

\footnotetext{
${ }^{3}$ To be precise, this calculation also includes a vertex correction beyond the usual $G W$.
}

The $G W$ approach thus yields very gratifying results concerning the band structure, but having a good band structure is not enough when one is interested in spectroscopies like absorption, where one creates a neutral, i.e., electron-hole type of excitation. One is in that case talking about the response of the system to a (timedependent) external potential, which implies that one is interested in the dielectric matrix itself, as defined in Eq. (1.1) in its obvious time-dependent extension. This response, when looked at from the point of view of the system in its ground state, implies a redistribution of charge (wave functions), in other words, depletion of charge (holes) or accumulation of charge (electrons) in some places. This creation of electron-hole pairs can be seen explicitly in the corresponding equations. In fact, in direct (r) space, the dielectric function for an electron system can be written as

$$
\varepsilon\left(\mathbf{r}, \mathbf{r}^{\prime}, \omega\right)=\delta\left(\mathbf{r}-\mathbf{r}^{\prime}\right)-\int d \mathbf{r}^{\prime \prime} v\left(\mathbf{r}-\mathbf{r}^{\prime \prime}\right) P\left(\mathbf{r}^{\prime \prime}, \mathbf{r}^{\prime}, \omega\right)
$$

where $v$ is the bare Coulomb interaction and where a polarizability operator $P\left(\mathbf{r}^{\prime \prime}, \mathbf{r}^{\prime}, \omega\right)$ has been introduced. When $P$ is zero, the system is not polarizable and hence the total potential is equal to the external one. Otherwise, $P$ is, in general and on average, negative, i.e., acting against the external potential. The simplest approximation for $P$ is the independent (quasi)particle form:

$$
P_{I Q P}\left(\mathbf{r}, \mathbf{r}^{\prime}, \omega\right):=\sum_{i j}\left(f_{i}-f_{j}\right) \frac{\psi_{i}(\mathbf{r}) \psi_{j}^{*}(\mathbf{r}) \psi_{j}\left(\mathbf{r}^{\prime}\right) \psi_{i}^{*}\left(\mathbf{r}^{\prime}\right)}{\omega-\omega_{i j}+i \eta} .
$$

Here $\omega_{i j}=\left(\epsilon_{j}-\epsilon_{i}\right), f_{i}$ are Fermi occupation numbers, and $(i, j)$ label the states of energy $\epsilon_{j}$ and $\epsilon_{i}$ obtained from some (for the moment, unspecified) equation for one-particle states. The small imaginary number in leads to an imaginary part of $P$ which is proportional to $\delta\left(\epsilon_{j}-\epsilon_{i}-\omega\right)$; in other words, one can see the energy conservation for a photon $\omega$ promoting an electron from state $i$ to state $j$.

The approximation for the polarizability $P_{I Q P}$ in Eq. (1.4) as a sum over independent transitions has the form of the random-phase approximation (RPA; Adler, 1962; Wiser, 1963). In fact, the RPA was originally meant to describe a calculation performed within the (linearized) time-dependent Hartree approach, or Lindhard approximation (Lindhard 1954), for a homogeneous electron gas. Later, the time-dependent Hartree approach was shown by Ehrenreich and Cohen (1959) to be equivalent to the diagrammatic bubble expansion for the dielectric function in many-body perturbation theory (see also Pines, 1963, and Pines and Nozières, 1989). Here, the term RPA form will in the following indicate that the approximation (1.4) has been used for $P$. The "additional" holes $i$ and electrons $j$ can of course be calculated within the framework of the one-particle excitations outlined above. It turns out that the dielectric function evaluated using Kohn-Sham orbitals $\psi_{i}$ and eigenvalues $\epsilon_{i}$ from LDA in Eq. (1.4) yields absorption spectra that are in quantitative, and sometimes even in qualitative, disagreement with experiments (Cohen and 
Chelikowsky, 1988). Since the factor $\delta\left(\epsilon_{j}-\epsilon_{i}-\omega\right)$ implies that absorption occurs at eigenvalue differences, this has been in part attributed to the fact that the KohnSham eigenvalues underestimate the quasiparticle gap, which leads to a redshift of the theoretical spectrum with respect to the experimental one. In fact, for example, in a small sodium cluster $\mathrm{Na}_{4}$ the first allowed transition between Kohn-Sham orbitals occurs at $1.1 \mathrm{eV}$ (Onida et al., 1995), whereas the first experimental absorption peak is seen at $1.8 \mathrm{eV}$ (Wang et al., 1990).

However, even when the quasiparticle gap problem is corrected by replacing the Kohn-Sham eigenvalues by quasiparticle energies calculated, for example, in the $G W$ approximation (this approach will be named $G W$-RPA in the following), important discrepancies with experiment are found. In general, the redshift problem becomes a blueshift problem-in the case of $\mathrm{Na}_{4}$, the first main absorption peak is shifted to $3.3 \mathrm{eV}$. This problem is not due to a failure of the $G W$ approach. In fact, for $\mathrm{Na}_{4}$ the ionization potential, i.e., the creation of a hole, is correctly described within $G W$ (Reining et al., 2000). In the latter case, however, one considers the addition of a hole with respect to the ground state and not, as in the case of absorption, the addition of a hole and then the addition of an electron in the presence of that hole. An additional correction term to the potential is hence needed in the case of absorption, expressed by the change of the potential upon presence of the hole, and a self-consistent treatment of hole and electron altogether. One has therefore to expect an effective two-particle equation for the response function, including electronhole interaction effects. This equation-the BetheSalpeter equation-is actually found via the rigorous treatment of the problem within Green's-function theory (see Sec. IV.B). It requires the correct calculation of the quasielectron and the quasihole (for example, via $G W$ ) and contains an interaction term that mixes the formerly independent transitions. The electron-hole interaction hence modifies the expression for the polarizability in Eq. (1.4). These excitonic effects have been well known for some time, starting from a description based on a superposition of atomic excitations (Frenkel, 1931a, 1931b; Peierls, 1932) and the insertion of the concept of "excitons" into the band picture (Wannier, 1937), and extending to detailed work in the 1950s like that of Heller and Marcus (1951), or of Haken (1956, 1957). An overview of the intense activity in the field at that time can be found in the book of Knox (1963).

Sham and Rice (1966) established the link between exciton models and many-body theory, by deriving the effective mass approximation from the Bethe-Salpeter equation. The first realistic nonmodel calculation for valence excitons in a solid based on the Bethe-Salpeter equation was performed by Hanke and Sham, first using the time-dependent Hartree-Fock approximation (which is equivalent to introducing an unscreened electron-hole interaction; Hanke and Sham 1974, 1975) and later also introducing a static electron-hole screening (Hanke and Sham, 1980). Their calculation, based on the linear combination of atomic orbitals, managed to explain the qualitative features of the line shape of the spectra of diamond and silicon. The $\mathrm{Na}_{4}$ cluster was the first realistic system to be treated in the $a b$ initio scheme starting from DFT and going through $G W$ (Onida et al., 1995), with a particularly strong effect of the electron-hole interaction due to the finite size of the cluster: the first absorption peak was moved back by $1.5 \mathrm{eV}$ from its $G W$ position, with a result close to the experimental one. An increasing number of such $a b$ initio calculations of excitonic effects in finite and extended systems (see, for example, Albrecht et al., 1997, 1998a, 1998b; Benedict et al., 1998a, 1998b; Rohlfing and Louie, 1998a, 1998b) have appeared since then, generally showing a big improvement over the results of RPA and $G W$-RPA calculations.

Of course these two-particle electron-hole calculations are relatively cumbersome, and it has always been tempting to stay within the framework of DFT. One could in fact consider the DFT exchange-correlation potential $V_{x c}$ as an approximation to the self-energy and, knowing that self-energy and electron-hole interaction effects partially cancel each other, ${ }^{4}$ hope that $V_{x c}$ together with its variation with density (the functional derivative $\delta V_{x c} / \delta \rho$, i.e., the so-called exchange-correlation kernel $f_{x c}$ ), could yield improved optical spectra. This resembles the theoretically more rigorous TDDFT (Runge and Gross, 1984; Gross and Kohn, 1985), where the response of the electrons to a time-dependent external potential is derived by searching the extrema of the quantum-mechanical action functional, which leads to a time-dependent Kohn-Sham equation. It has turned out that TDDFT in the adiabatic local-density approximation (TDLDA) often yields good spectra for finite systems. For $\mathrm{Na}_{4}$ and other sodium clusters, Vasiliev et al. (1999) have calculated absorption spectra in excellent agreement with experiment, i.e., reproducing peak positions and relative heights within $\simeq 10 \%(0.2 \mathrm{eV})$. This is not in contradiction with the fact that, as mentioned above, the Kohn-Sham eigenvalue differences are smaller than the absorption energies: TDDFT directly describes the evolution of the density under the influence of a time-dependent external potential, which means that both the potential (yielding the Kohn-Sham eigenvalues) and variations of the potential (which can shift the excitation energies) are taken into account. TDLDA is also successful in describing electron-energyloss spectra for bulk metals and semiconductors, but improves only very slightly the absorption spectra of solids with respect to RPA calculations (Gavrilenko and Bechstedt, 1996). Therefore, in the field of TDDFT the main effort is directed toward finding better approximations for both the exchange-correlation potential and its variation with the density (i.e., $f_{x c}$ ). To this end, one has to understand the main differences between finite and infinite systems, as well as between different spectroscopies.

\footnotetext{
${ }^{4}$ See the example of $\mathrm{Na}_{4}$ in Onida et al. (1995), and the results of the jellium model for metal clusters by Pacheco and Ekardt (1997; also Ekardt and Pacheco, 1995).
} 
In fact, for example, absorption and electron- energyloss spectra are derived in different ways from the same dielectric function.

In the following we shall therefore concentrate in more detail on the dielectric function and its link to spectroscopy. In particular, we shall outline those features that are common to both TDDFT and BetheSalpeter approaches, before treating the two approaches separately and finally comparing them.

\section{TDDFT AND GREEN'S-FUNCTION APPROACHES: COMMON INGREDIENTS}

TDDFT is an extension of static ground-state densityfunctional theory. Also, Green's-function calculations often start from DFT results. In fact, concerning the ground state, calculations based on density-functional theory using simple density functionals predominate today. Current DFT results have often surpassed those from standard ab initio quantum chemistry techniques like Hartree-Fock, configuration interaction, etc., which may require heavy compromises in their technical realization for systems that are not very small (e.g., full configuration-interaction calculations can hardly be done for more than five electrons; Jensen, 1999). Despite the failures of DFT due to the approximations made for the exchange-correlation contributions, its use continues to increase because of its wide applicability and its favorable scaling with the number of atoms.

In the following, we briefly review the basic ingredients of DFT in view of its use as a starting point for spectroscopy calculations. For a more detailed description we refer the reader to any of the numerous compilations published in recent years, e.g., Lundqvist and March (1983); Dreizler and Gross (1990); Seminario (1996).

\section{A. Density-functional theory for the ground state}

The ground-state energy of a system of interacting electrons in an external potential can be written as a functional of the ground-state electronic density. Compared to conventional quantum-chemistry methods this approach is particularly appealing, since it does not rely on a complete knowledge of the $N$-electron wave function but only on the electronic density. Of course, although the theory is exact, the energy functional is unknown and has to be approximated in practical implementations. Today's DFT starts with the theorems of Hohenberg and Kohn (1964) for a search of the electronic ground state of an isolated system of $N$ interacting electrons in an external potential $V_{e x t}(\mathbf{r})$. The first theorem ("the density as the basic variable in the electronic problem") establishes that the external potential $V_{\text {ext }}(\mathbf{r})$ is a functional of the charge density $\rho(\mathbf{r})$, within an additive constant. The second theorem establishes the "energy variational principle for the density." These two theorems show that the problem of solving the many-body Schrödinger equation for the ground state can be exactly recast into the variational problem of minimizing the Hohenberg-Kohn functional with respect to the charge density.

In practice, the available approximations for kinetic energy and exchange-correlation density-based functionals give moderate quantitative agreement with experimental data (Dreizler and Gross, 1990). A much improved strategy has been presented by Kohn and Sham (1965) using orbital variables. The fundamental assumption is to introduce a reference system of noninteracting electrons in an external potential $V_{\text {eff }}^{K S}(\mathbf{r})$ such that the ground-state charge density of the physical system coincides with that of the reference system. ${ }^{5}$ With the introduction of the noninteracting system, the variational problem on $\rho(\mathbf{r})$ is thus finally reformulated in terms of the following set of self-consistent Kohn-Sham equations:

$$
\left[-\frac{1}{2} \nabla^{2}+V_{H}(\mathbf{r})+V_{x c}(\mathbf{r})+V_{0}(\mathbf{r})\right] \psi_{i}(\mathbf{r})=\varepsilon_{i} \psi_{i}(\mathbf{r})
$$

with the density given by $\rho(\mathbf{r})=\sum_{i=1}^{N}\left|\psi_{i}(\mathbf{r})\right|^{2}$. Thus $V_{\text {eff }}^{K S}$ $=V_{H}+V_{x c}+V_{0}$, where $V_{H}$ is the Hartree potential, $V_{x c}(\mathbf{r})=\delta E_{x c}[\rho] / \delta \rho(\mathbf{r})$ is the exchange-correlation potential that contains all many-body effects, and $V_{0}$ is an external potential stemming, for example, from the electron-ion interaction.

The simplest approximation (still widely used) to $E_{x c}$ is the local-density approximation (Kohn and Sham, 1965). The approximation is based on using the exchange-correlation energy density $\epsilon_{x c}^{\text {hom }}$ of the homogeneous electron gas (Ceperley and Alder, 1980; Ortiz et al., 1999), namely, $E_{x c}^{L D A}[\rho]=\int \epsilon_{x c}^{h o m}(\rho(\mathbf{r})) \rho(\mathbf{r}) d \mathbf{r}$. Hence one replaces the inhomogeneous electron system at each point $\mathbf{r}$ by a homogeneous electron gas having the density of the inhomogeneous system at $\mathbf{r}$. The rationale for this approximation is in the limit of slowly varying density. Unexpectedly, the domain of applicability of the LDA has been found to go much beyond the nearly free-electron gas and accurate results can be obtained for very inhomogeneous systems. Improvements over the LDA have been found, e.g., by the generalizedgradient approximations, in which the exchangecorrelation energy density is a function not only of the electron density, but also of its gradient (see, e.g., Perdew et al., 1996). Further improvements over the generalized-gradient functional should go in the direction of nonlocal functionals, in order to describe better the inhomogeneity of the exchange-correlation hole, as in the case of the exact exchange potentials (Gross et al., 1996).

The issue of calculating electronic excitations, however, goes beyond the problem of finding a good approximation to the ground-state exchange-correlation

\footnotetext{
${ }^{5}$ This definition is meaningful if the charge density is noninteracting $v$ representable, so that one has a unique definition of the total energy Kohn-Sham functional.
} 
functional. The problem is intimately linked to the fact that the one-particle eigenvalues in the Kohn-Sham theory have often been used, as well, to discuss the excitation spectra of solids, molecules and atoms. Although there is no rigorous justification for a direct identification of Kohn-Sham eigenvalues with quasiparticle energies, the formal resemblance between Kohn-Sham and quasiparticle equations, such as the COHSEX equation [Eq. (1.2)], where $\Sigma\left(\mathbf{r}, \mathbf{r}^{\prime}, \omega\right)$ replaces $\delta(\mathbf{r}$ $\left.-\mathbf{r}^{\prime}\right) V_{x c}(\mathbf{r})$ in Eq. (2.1) explains why the Kohn-Sham equation can be considered at least as a starting point.

As pointed out above, once some one-particle-like theory such as Kohn-Sham has been established, response functions and spectra can be constructed, for example in the approximation (1.4). This is the subject of the next section.

\section{B. The inverse dielectric function and local-field effects}

As discussed above, information about the electrons and holes, and about their interaction, is contained in the dielectric function via the polarizability $P$ of Eq. (1.3). However, further considerations are necessary in order to obtain spectra. In fact, independently of how $P$ and $\varepsilon$ are calculated, the latter usually has to be inverted, since one is concerned with the total potential $V_{t o t}=\varepsilon^{-1} V_{e x t}$ for a given external potential $V_{e x t}$, and not vice versa. Equivalently, the induced charge is given by $\rho_{\text {ind }}=\chi V_{\text {ext }}$, where the reducible polarizability $\chi$ and $\varepsilon^{-1}$ are linked by

$\varepsilon^{-1}\left(\mathbf{r}, \mathbf{r}^{\prime}, \omega\right)=\delta\left(\mathbf{r}-\mathbf{r}^{\prime}\right)+\int d \mathbf{r}^{\prime \prime} v\left(\mathbf{r}-\mathbf{r}^{\prime \prime}\right) \chi\left(\mathbf{r}^{\prime \prime}, \mathbf{r}^{\prime}, \omega\right)$.

In the following, it is useful to describe explicitly two types of spectra containing neutral excitations, namely, photon absorption and electron-energy-loss spectra (EELS). In electron-energy-loss experiments, an electron impinges on the sample and loses energy by exciting electron-hole pairs, plasmons, and other high-order multipair excitations. This energy loss is given by the imaginary part of the integral of the potential created by the electron, and the induced charge. When the potential $V_{\text {ext }}$ due to an electron is taken proportional to a plane wave, this leads, for a momentum transfer $\mathbf{q}$, to the loss function

$$
L(\omega) \propto-\operatorname{Im}\left(\int d \mathbf{r} d \mathbf{r}^{\prime} e^{-i \mathbf{q r}} \chi\left(\mathbf{r}, \mathbf{r}^{\prime}, \omega\right) e^{i \mathbf{q} \mathbf{r}^{\prime}}\right),
$$

which is hence determined by the Fourier transform of the inverse dielectric function $-\operatorname{Im}\left[\varepsilon^{-1}(\mathbf{q}, \mathbf{q}, \omega)\right]$.

In a finite system, $\chi$ also yields the photoabsorption cross section $\sigma$, via

$$
\sigma(\omega)=\frac{4 \pi \omega}{c} \operatorname{Im} \alpha(\omega),
$$

where $c$ is the velocity of light and $\operatorname{Im} \alpha(\omega)$ is the imaginary part of the dynamical polarizability,

$\alpha(\omega)=-\int d \mathbf{r} d \mathbf{r}^{\prime} V_{e x t}(\mathbf{r}, \omega) \chi\left(\mathbf{r}, \mathbf{r}^{\prime}, \omega\right) V_{e x t}\left(\mathbf{r}^{\prime}, \omega\right)$.
In particular, for a dipolar external field $V_{\text {ext }}$ along the $z$ direction, the corresponding component of the $\sigma$ tensor reads

$$
\sigma_{z z}(\omega)=-\frac{4 \pi \omega}{c} \operatorname{Im} \int d \mathbf{r} d \mathbf{r}^{\prime} z \chi\left(\mathbf{r}, \mathbf{r}^{\prime}, \omega\right) z^{\prime},
$$

which can also be obtained from the long-wavelength limit of $\varepsilon^{-1}: \quad \sigma(\omega)=\lim _{\mathbf{q} \rightarrow 0}(\omega / c) \operatorname{Im}[v(\mathbf{q}) \chi(\mathbf{q}, \mathbf{q}, \omega)]$ along the field direction. The other components of the tensor can be similarly obtained. Note that in molecularbeam experiments, where the molecules are randomly oriented, one measures the trace of the tensor, that is, $\sigma_{\exp }(\omega)=\frac{1}{3} \Sigma_{i=(x, y, z)} \sigma_{i i}(\omega)$.

In the solid, one has to distinguish between the loss spectra and the absorption spectra, which are instead described by the macroscopic dielectric function $\varepsilon_{M}$. Its relation to the microscopic dielectric function $\varepsilon$ of periodic crystals has been discussed by Adler (1962) and Wiser (1963) as well as by Ehrenreich (1966):

$$
\varepsilon_{M}(\omega) \equiv \lim _{\mathbf{q} \rightarrow 0} \frac{1}{\varepsilon_{\mathbf{G}=0, \mathbf{G}^{\prime}=0}^{-1}(\mathbf{q}, \omega)},
$$

where $\varepsilon_{\mathbf{G G}^{\prime}}(\mathbf{q}, \omega):=\varepsilon\left(\mathbf{q}+\mathbf{G}, \mathbf{q}+\mathbf{G}^{\prime}, \omega\right)$ is the Fourier transform to reciprocal space of $\varepsilon\left(\mathbf{r}, \mathbf{r}^{\prime}\right), \mathbf{G}$ is a reciprocal lattice vector, and $\mathbf{q}$ belongs to the first Brillouin zone. The optical absorption spectrum is then given by the imaginary part $\varepsilon_{2}(\omega)$ of $\varepsilon_{M}(\omega)$. The dielectric constant $\varepsilon_{0}$ is the value of $\varepsilon_{M}(\omega)$ at $\omega=0$. If $\varepsilon$ is diagonal in $\mathbf{G}, \mathbf{G}^{\prime}, \varepsilon_{M}$ is just $\varepsilon_{M}=\lim _{\mathbf{q} \rightarrow 0} \varepsilon_{\mathbf{G}=0, \mathbf{G}^{\prime}=0}$, i.e., the spatial average of the microscopic dielectric function. This is in fact the case in the homogeneous electron gas. Otherwise, the microscopic dielectric function $\varepsilon\left(\mathbf{r}, \mathbf{r}^{\prime}\right)$ will depend explicitly on the positions $\mathbf{r}$ and $\mathbf{r}^{\prime}$, and not simply on the distance $\left|\mathbf{r}-\mathbf{r}^{\prime}\right|$. This is translated into the fact that the dielectric matrix in reciprocal space is not diagonal. The fact that all elements of the matrix contribute to one element of its inverse reflects the so-called local field effects: these effects arise whenever the system under study is nonhomogeneous on the microscopic scale. In this case, for example, an external spatially constant perturbing field will induce fluctuations on the scale of the interatomic distances in the material, giving rise to additional internal microscopic fields. These concepts will be helpful in the next section [see the discussion after Eq. (2.23)], and in Sec. VI, where TDDFT and Bethe-Salpeter methods are compared, outlining the equations and the part of the kernel which are common to both approaches (see in particular Sec. VI.D.1). It is clear that the local field effects should in principle be included in both absorption and loss spectra, since the crucial quantity which appears is always the inverse dielectric function.

Local field effects can shift peak positions, and they can be very important, as will be discussed later. However, even when local field effects are taken into account, the above conclusions about the need to go beyond RPA or $G W$-RPA in the calculation of $P$, and hence $\varepsilon$, remain valid (for example, the inclusion of local field effects does not generally lead to a significant im- 
provement of the absorption spectra of solids; Louie et al., 1975). It should in fact be stressed that the concept of local field effects is independent of the approach used to calculate $P$ and $\varepsilon$. It is therefore useful to summarize here the notations that will be used throughout this review in order to characterize the different levels of approximation: First, RPA and $G W$-RPA refer to a calculation in which $P$ has been approximated by the form (1.4), using DFT and $G W$ eigenvalues, respectively, but the spectrum has then correctly been constructed from $\varepsilon^{-1}$. When local field effects are neglected in the inversion, in the dipole limit Eq. (1.4) leads to the well-known Ehrenreich and Cohen (1959) expression for the imaginary part of the macroscopic dielectric function in terms of the matrix elements of the velocity operator $\mathbf{v}$ between valence and conduction states: ${ }^{6}$

$\operatorname{Im}\left\{\varepsilon_{M}(\omega)\right\}=\frac{16 \pi}{\omega^{2}} \sum_{v, c}\left|\left\langle\psi_{v}|\mathbf{v}| \psi_{c}\right\rangle\right|^{2} \delta\left(\epsilon_{c}-\epsilon_{v}-\omega\right)$.

Independently of the quality of the states $\psi$ entering Eq. (1.4), the electronic excitations described by Eq. (2.8) are restricted to the generation of noninteracting particle-hole pairs. In the following this approximation will be called an independent-particle-random-phase approximation macroscopic dielectric function (Del Sole and Girlanda, 1993).

It is also interesting to point out that, on the other hand, if the matrix inversion is properly taken into account, the formerly independent transitions mix. In other words, even if no electron-hole interaction is included in $P$, there is an effective electron-hole interaction showing up in $\varepsilon_{M}$. This is actually an electron-hole exchange term, as will be discussed later.

The inclusion of local field effects is, in principle, straightforward: Inverting $\varepsilon$ and placing the Fourier transform of the inverse dielectric function in Eq. (2.7) directly yields the macroscopic dielectric function for each frequency $\omega$. For our purpose, however, it is convenient to use a different formulation for the macroscopic dielectric function, which is suitable for a subsequent inclusion of excitonic effects. Moreover, and more importantly here, it will enable us to compare in a concise way (i) electron-energy-loss and absorption spectra and, later, (ii) TDDFT and the Bethe-Salpeter approach. In fact, one can show (see Hanke, 1978 and Appendix B.1) that $\varepsilon_{M}$ can be constructed from a modified response function $\bar{P}$,

$$
\varepsilon_{M}(\omega)=1-\lim _{\mathbf{q} \rightarrow 0}\left[v(\mathbf{q})_{0} \bar{P}_{\mathbf{G}=\mathbf{G}^{\prime}=0}(\mathbf{q}, \omega)\right],
$$

where the matrix $\bar{P}_{\mathbf{G}, \mathbf{G}^{\prime}}$ satisfies the Dyson-like screening equation

$$
\bar{P}=P+P \bar{v} \bar{P} .
$$

\footnotetext{
${ }^{6}$ This is none other than Fermi's golden rule in the dipole approximation and the one-electron picture for absorption processes (Pines, 1963; Fetter and Walecka, 1971).
}

Here we have defined the modified Coulomb interaction $\bar{v}(\mathbf{q})_{\mathbf{G}}$ as follows:

$$
\bar{v}(\mathbf{q})_{\mathbf{G}}=\left\{\begin{array}{cc}
0 & \text { if } G=0 \\
v(\mathbf{q})_{\mathbf{G}}=\frac{4 \pi}{|\mathbf{q}+\mathbf{G}|^{2}} & \text { else }
\end{array}\right\} .
$$

In other words, the difference between $\bar{v}$ and $v$ is "only" in the $\mathbf{G}=0$ component. $^{7}$ Because of the long range of the Coulomb interaction, this term is divergent for vanishing q, which explains its importance, as will be discussed below. $\bar{v}$, on the other hand, does not diverge and plays the role of a correction term, namely, the local field effects. In fact, from Eq. (2.10) it is clear that putting $\bar{v}$ to zero is equivalent to neglecting those effects.

It is interesting to compare Eq. (2.10) to the corresponding Dyson-like equation for $\chi$, which follows from Eqs. (2.2) and (1.3):

$$
\chi=P+P v \chi,
$$

the only difference is in fact the $\mathbf{G}=0$ part of the bare Coulomb interaction. This remark turns out to be extremely important, as will be discussed later in the comparison of TDDFT and Bethe-Salpeter results for finite and infinite systems. One can put the common term $\bar{v}$ into evidence by writing Eq. (2.11) as

$$
\begin{aligned}
\chi_{\mathbf{G}, \mathbf{G}^{\prime}}= & P_{\mathbf{G}, \mathbf{G}^{\prime}} \\
& +\sum_{\mathbf{G}^{\prime \prime}} P_{\mathbf{G}, \mathbf{G}^{\prime \prime}} \overline{\boldsymbol{v}}_{\mathbf{G}^{\prime \prime}} \chi_{\mathbf{G}^{\prime \prime}, \mathbf{G}^{\prime}}+P_{\mathbf{G}, 0} v_{0} \chi_{0, \mathbf{G}^{\prime}} .
\end{aligned}
$$

One can then immediately write down Eq. (2.12) and (2.10) for the case when local field effects are neglected, and take the macroscopic limit; two quantities $\bar{P}_{00}=P_{00}$ and $\chi_{00}=P_{00} /\left(1-P_{00} v_{0}\right)$ are obtained, which describe, respectively, the absorption and the electron-energy-loss spectra of the system. Also in this simplified case it is clear that $\chi$ and $\bar{P}$ are fundamentally different $(\chi$ is screened, but $\bar{P}$ is not), and that this fact is entirely due to the seemingly tiny difference of the kernel of the Dyson-like equations, i.e., the difference between $v$ and $\bar{v}$ (see detailed discussions and applications in Sec. VI.D).

Until now, all considerations have been general, i.e., $P$ has not been specified. Only when talking explicitly about $P$ will there be a difference between TDDFT and the Bethe-Salpeter approach. However, in spite of-or because of-the differences that will arise, it is worthwhile to anticipate a qualitative discussion of the structure of the equations leading to the determination of $P$. This allows one to highlight the importance and conse-

\footnotetext{
${ }^{7}$ In a finite system, one can still have a wave vector $\mathbf{k}$ as a sum of a reciprocal lattice vector $\mathbf{G}$ and a vector $\mathbf{q}$ lying inside the first Brillouin zone, by creating a periodic array of "unit cells" of vacuum, each of them containing a copy of the system. The limit of infinite volume (isolated systems) for those supercells corresponds to the zero-volume limit for the first Brillouin zone, i.e., to an infinitely dense $\mathbf{G}$-space.
} 
quences of induced local and nonlocal potentials, without masking simple concepts by complicated many-body formulations. For the case of Hartree-Fock, many details can be found in the reviews of McLachlan and Ball (1964) and Langhoff et al. (1972).

\section{Four-point kernels and effective two-particle equations}

An effective self-consistent Hamiltonian, which is made time dependent through the time dependence of the wave functions, in the presence of a time-dependent external potential, allows one to calculate the induced density matrix to first order (Hedin and Lundqvist, 1969):

$$
\begin{aligned}
\rho_{\text {ind }}\left(\mathbf{r}, \mathbf{r}^{\prime}, \omega\right)= & \sum_{n, n^{\prime}} \psi_{n}(\mathbf{r}) \\
& \times \frac{\left(f_{n}-f_{n^{\prime}}\right)\left\langle n\left|H^{(1)}(\omega)\right| n^{\prime}\right\rangle}{\epsilon_{n}-\epsilon_{n^{\prime}}-\omega} \psi_{n^{\prime}}^{*}\left(\mathbf{r}^{\prime}\right),
\end{aligned}
$$

where $\epsilon_{n}$ and $\psi_{n}(\mathbf{r})=\langle\mathbf{r} \mid n\rangle$ are the eigenvalues and eigenfunctions of the unperturbed Hamiltonian. Then $\chi$ is determined through

$$
\rho_{\text {ind }}(\mathbf{r}, \mathbf{r})=\int d \mathbf{r}^{\prime} \chi\left(\mathbf{r}, \mathbf{r}^{\prime}\right) V_{\text {ext }}\left(\mathbf{r}^{\prime}\right) .
$$

The perturbation $H^{(1)}$ not only is given by the external potential $V_{\text {ext }}$, but also contains self-consistently the induced potential, which is proportional to the induced density matrix (schematically $V_{\text {ind }}=\rho_{\text {ind }} \delta V_{\text {eff }} / \delta \rho$ ).

A typical effective Hamiltonian will have an effective potential $V_{\text {eff }}\left(\mathbf{r}_{1}, \mathbf{r}_{2}, t\right)$ that has some contributions that are local and depend on the density, i.e., $V_{\text {loc }}\left\{[\rho(\widetilde{\mathbf{r}}, \widetilde{t})], \mathbf{r}_{1}, t\right\} \delta\left(\mathbf{r}_{1}-\mathbf{r}_{2}\right)$ (like the Hartree potential or the DFT exchange-correlation potential), and others that are nonlocal but directly proportional to the density matrix (like the Hartree-Fock exchange potential): $\rho\left(\mathbf{r}_{1}, \mathbf{r}_{2}, t\right) w\left(\mathbf{r}_{1}, \mathbf{r}_{2}\right)$, where $w\left(\mathbf{r}_{1}, \mathbf{r}_{2}\right)$ is some generalized interaction. For simplicity here we do not consider more complicated time-dependent interactions, but include the possibility of memory effects in $V_{l o c}$, i.e., $V_{l o c}$ can be a functional of the density at all (past) times $\widetilde{t}$. In this case, the induced potential is

$$
\begin{aligned}
V_{\text {ind }}\left(\mathbf{r}_{1}, \mathbf{r}_{2}, t\right)= & \int d \mathbf{r}_{3} d \mathbf{r}_{4} d t^{\prime}\left[\delta\left(\mathbf{r}_{1}-\mathbf{r}_{2}\right) \delta\left(\mathbf{r}_{3}-\mathbf{r}_{4}\right)\right. \\
& \times \frac{\delta V_{l o c}\left([\rho(\widetilde{\mathbf{r}}, \widetilde{t})], \mathbf{r}_{1}, t\right)}{\delta \rho\left(\mathbf{r}_{3}, \mathbf{r}_{3}, t^{\prime}\right)} \\
& \left.+\delta\left(\mathbf{r}_{1}-\mathbf{r}_{3}\right) \delta\left(\mathbf{r}_{2}-\mathbf{r}_{4}\right) w\left(\mathbf{r}_{1}, \mathbf{r}_{2}\right) \delta\left(t-t^{\prime}\right)\right] \\
& \times \rho_{\text {ind }}\left(\mathbf{r}_{3}, \mathbf{r}_{4}, t^{\prime}\right) \\
:= & \int d \mathbf{r}_{3} d \mathbf{r}_{4} d t^{\prime} K\left(\mathbf{r}_{1}, \mathbf{r}_{2}, \mathbf{r}_{3}, \mathbf{r}_{4}, t, t^{\prime}\right) \\
& \times \rho_{\text {ind }}\left(\mathbf{r}_{3}, \mathbf{r}_{4}, t^{\prime}\right),
\end{aligned}
$$

which defines the four-point kernel $K$. Equations (2.13) and (2.15) can be put into a closed form for the induced density matrix, by introducing the four-point function ${ }^{4} \chi_{0}$ [see Eq. (B16)] such that Eq. (2.13) becomes

$$
\begin{aligned}
\rho_{\text {ind }}\left(\mathbf{r}_{1}, \mathbf{r}_{2}, \omega\right)= & \int d \mathbf{r}_{3} d \mathbf{r}_{4} d \mathbf{r}_{5} d \mathbf{r}_{6} \\
& \times\left(1-{ }^{4} \chi_{0} K\right)^{-1}\left(\mathbf{r}_{1}, \mathbf{r}_{2}, \mathbf{r}_{3}, \mathbf{r}_{4}, \omega\right) \\
& \times{ }^{4} \chi_{0}\left(\mathbf{r}_{3}, \mathbf{r}_{4}, \mathbf{r}_{5}, \mathbf{r}_{6}, \omega\right) \\
& \times V_{\text {ext }}\left(\mathbf{r}_{5}, \omega\right) \delta\left(\mathbf{r}_{5}-\mathbf{r}_{6}\right) .
\end{aligned}
$$

Hence from Eq. (2.14)

$$
\begin{aligned}
\chi\left(\mathbf{r}_{1}, \mathbf{r}_{2}, \omega\right)= & \int d \mathbf{r}_{3} d \mathbf{r}_{4}\left(1-{ }^{4} \chi_{0} K\right)^{-1}\left(\mathbf{r}_{1}, \mathbf{r}_{1}, \mathbf{r}_{3}, \mathbf{r}_{4}, \omega\right) \\
& \times{ }^{4} \chi_{0}\left(\mathbf{r}_{3}, \mathbf{r}_{4}, \mathbf{r}_{2}, \mathbf{r}_{2}, \omega\right) .
\end{aligned}
$$

An equation of this kind is Eq. (4.9), derived below for the particle-hole response function in the BetheSalpeter scheme. Another one is the TDDFT equation. Note that in this case, since there are only local potentials, the $\delta$ functions in the kernel (2.15) allow us to contract all equations immediately and to work with two-point functions from the beginning (see, e.g., Bertsch and Tsai, 1975; Bertsch and Broglia, 1994). In particular, the four-point function ${ }^{4} \chi_{0}$ can immediately be replaced by the two-point function $P_{I Q P}\left(\mathbf{r}_{1}, \mathbf{r}_{2}\right)$ $={ }^{4} \chi_{0}\left(\mathbf{r}_{1}, \mathbf{r}_{1}, \mathbf{r}_{2}, \mathbf{r}_{2}\right)$ defined in Eq. (1.4), as in the main equation of TDDFT [Eq. (5.11)] in Sec. V.B. A comparison of Eq. (2.17) and the four-point generalization of Eq. (2.11) allows one to get the four-point polarizability ${ }^{4} P$ from the equation

$$
{ }^{4} P={ }^{4} \chi_{0}+{ }^{4} \chi_{0}{ }^{4} f{ }^{4} P
$$

with

$$
{ }^{4} f\left(\mathbf{r}_{1}, \mathbf{r}_{2}, \mathbf{r}_{3}, \mathbf{r}_{4}\right)=K\left(\mathbf{r}_{1}, \mathbf{r}_{2}, \mathbf{r}_{3}, \mathbf{r}_{4}\right)-{ }^{4} v\left(\mathbf{r}_{1}, \mathbf{r}_{2}, \mathbf{r}_{3}, \mathbf{r}_{4}\right),
$$

and $^{8}$

$$
{ }^{4} v\left(\mathbf{r}_{1}, \mathbf{r}_{2}, \mathbf{r}_{3}, \mathbf{r}_{4}\right)=\delta\left(\mathbf{r}_{1}-\mathbf{r}_{2}\right) \delta\left(\mathbf{r}_{3}-\mathbf{r}_{4}\right) v\left(\mathbf{r}_{1}, \mathbf{r}_{3}\right) .
$$

The two-point $P$ is then

$$
P\left(\mathbf{r}_{1}, \mathbf{r}_{2}\right)={ }^{4} P\left(\mathbf{r}_{1}, \mathbf{r}_{1}, \mathbf{r}_{2}, \mathbf{r}_{2}\right) .
$$

Using the Green's-function formalism which will be introduced below (Sec. III), $\chi_{0}$ and ${ }^{4} \chi_{0}$ will be expressed, respectively, as $\quad-i G_{0}(12) G_{0}(21)$ and $-i G_{0}(13) G_{0}(42)$.

In general, $V_{\text {eff }}$ will of course contain the Hartree potential, and hence $K$ will contain the variation of the Hartree potential with respect to the density, $\delta V_{H} / \delta \rho$, which is just the contribution $v$ appearing in the Dysonlike equation (2.11). This allows one to see the relation between the local field effects (see Sec. II.B) and the density variation of the Hartree potential: When local field effects are neglected, all spatial frequencies of the

\footnotetext{
${ }^{8}$ The definition of ${ }^{4} \bar{v}$, which is used in the four-point equations involving $\bar{P}$, is analogous to that of ${ }^{4} v$.
} 
induced Hartree potential are neglected in the Dysonlike equation apart from the spatial frequency given by the external potential, i.e., apart from the $\mathbf{G}=0$ contribution when the external potential is macroscopic. ${ }^{9}$ In finite systems, macroscopic potentials do not play a role, and the distinction is meaningless-neglecting local field effects becomes equivalent to completely neglecting the induced Hartree potential. Therefore Eq. (2.6) is at the same time proportional to the macroscopic loss function and the to limit of Eq. (2.9) for the case of an infinitely dense $\mathbf{G}$ space.

To summarize, one will in general find a four-point equation for $S={ }^{4} \chi$ or $S={ }^{4} \bar{P}$ :

$$
S={ }^{4} \chi_{0}+{ }^{4} \chi_{0} K S
$$

where $K$ contains $(K=v+f)$ or not $(K=\bar{v}+f)$ the long-range term $v(\mathbf{G}=0)$, depending on whether $S={ }^{4} \chi$ or $S={ }^{4} \bar{P}$. Any density-matrix-dependent effective potential will lead to a four-point equation for $S$. In order to get the two-point functions, $S$ has to be contracted like in the expression for the macroscopic dielectric function, given by

$$
\begin{aligned}
\varepsilon_{M}(\omega)= & -\lim _{\mathbf{q} \rightarrow 0} \\
& \times\left[v(\mathbf{q}) \int d r d r^{\prime} e^{-i \mathbf{q} \cdot\left(\mathbf{r}-\mathbf{r}^{\prime}\right){ }^{4}} \bar{P}\left(\mathbf{r}, \mathbf{r}, \mathbf{r}^{\prime}, \mathbf{r}^{\prime} ; \omega\right)\right]
\end{aligned}
$$

If no nonlocal terms are present in $V_{\text {eff }}$ (e.g., when $V_{\text {eff }}$ $\left.=V_{\text {Hartree }}+V_{x c}^{D F T}\right)$, Eq. $(2.20)$ can be contracted immediately, and one can work with two-point functions only.

Generally, in order to solve Eq. (2.20), one has to invert a four-point function for each frequency. This has been done even for solids, for example in the case of a Fock term in the effective Hamiltonian, in the early work of Hanke and Sham $(1974,1975)$. However, for a comparison between TDDFT and the Bethe-Salpeter equation method it is more convenient to present the equations in a different way, also often used. This scheme, described in the following, has the advantage of putting the two-particle nature of the problem into evidence. In fact, it has been shown (see Appendix B.2) that the four-point equation for $S$ can be transformed to an eigenvalue problem involving the effective twoparticle Hamiltonian:

$$
\begin{aligned}
H_{\left(n_{1} n_{2}\right),\left(n_{3} n_{4}\right)}^{2 p} \equiv & \left(\epsilon_{n_{2}}-\epsilon_{n_{1}}\right) \delta_{n_{1} n_{3}} \delta_{n_{2} n_{4}} \\
& +\left(f_{n_{1}}-f_{n_{2}}\right) K_{\left(n_{1} n_{2}\right),\left(n_{3} n_{4}\right)} .
\end{aligned}
$$

The indices $n_{i}$ refer to the fact that matrix elements have been taken with respect to four eigenfunctions of the starting effective static one-particle Hamiltonian. Hence $H^{2 p}$ is diagonalized, and from its eigenvalues $E_{\lambda}$

\footnotetext{
${ }^{9}$ The local field effects can also be included through a direct calculation of the variation of the Hartree potential in response to an electric field as done in density functional perturbation theory (Baroni et al., 2001 and references therein).
}

and eigenstates $A_{\lambda}^{n_{1} n_{2}}$ the four- and two-point quantities of interest are constructed. For example, the macroscopic dielectric function is given by

$$
\begin{aligned}
\varepsilon_{M}(\omega)= & 1-\lim _{\mathbf{q} \rightarrow 0} v(\mathbf{q}) \sum_{\lambda, \lambda^{\prime}}\left(\sum_{n_{1}, n_{2}}\left\langle n_{1}\left|e^{-i \mathbf{q} \cdot \mathbf{r}}\right| n_{2}\right\rangle\right. \\
& \times \frac{A_{\lambda}^{\left(n_{1} n_{2}\right)}}{\omega-E_{\lambda}+i \eta} N_{\lambda, \lambda^{\prime}}^{-1} \\
& \left.\times \sum_{n_{3}, n_{4}}\left\langle n_{4}\left|e^{i \mathbf{q} \cdot \mathbf{r}^{\prime}}\right| n_{3}\right\rangle A_{\lambda^{\prime}}^{*\left(n_{3} n_{4}\right)}\left(f_{n_{3}}-f_{n_{4}}\right)\right) .
\end{aligned}
$$

Here $N_{\lambda, \lambda^{\prime}}$ is an overlap matrix (see Appendix B.2). Since it can be shown that the $n_{i}$ always appear as pairs consisting of one occupied and one empty state (see Appendix B.2), this reflects the very physical approach of rewriting the problem in terms of mixing between different independent-particle transitions between occupied and unoccupied eigenstates of a one-particle Hamiltonian. Equation (2.23) should be compared to Eq. (2.8); the difference is caused by a change in excitation energies [from $\left(\epsilon_{c}-\epsilon_{v}\right)$ to $E_{\lambda}$ ], but also, very importantly, by the coefficients $A_{\lambda}$ which mix the formerly independent transitions. As pointed out above, even if only the Hartree potential is used in the derivation of $K$, i.e., if one considers only local field effects and no further exchange-correlation contributions, the electron-hole Hamiltonian is not diagonal, and the coefficients $A_{\lambda}$ will mix the transitions. This way of formulating the problem is hence also appealing because the knowledge of the $A_{\lambda}$ allows one to interpret spectra in terms of mixing of transitions.

One can thus define a general two-particle Hamiltonian which (i) yields, depending on its interaction potential, the inverse dielectric function or the macroscopic one and (ii) does so for systems that are described by an effective one-particle Hamiltonian either depending on just the density, or containing more complicated functionals involving the density matrix.

The corresponding equations have been derived starting from the time-dependent density matrix in Eq. (2.13). In order to talk now in detail about the possible effective one-particle Hamiltonians and derived kernels, one is naturally led to rigorously introduce in the following a sort of time-dependent density matrix for electrons and holes, which will allow the effective Hamiltonian and its derived response functions to be obtained on the same footing; one is led to introduce the Green's function. ${ }^{10}$

\footnotetext{
${ }^{10}$ Note that the history of modern band-structure calculations started with the Hartree theory, where the essential ingredient is the electronic density, $\rho(\mathbf{r})=\Sigma_{s} \psi_{s}^{*}(\mathbf{r}) \psi_{s}(\mathbf{r})$. Exchange effects (Hartree-Fock) are then included through the nonlocal density matrix $\rho\left(\mathbf{r}, \mathbf{r}^{\prime}\right)=\Sigma_{s} \psi_{s}^{*}\left(\mathbf{r}^{\prime}\right) \psi_{s}(\mathbf{r})$, whereas to introduce a time dependence, the natural way is to do it in the phase of the wave functions, which leads to a time-dependent density matrix: $\rho\left(\mathbf{r}, \mathbf{r}^{\prime}, t^{\prime}-t\right)=\Sigma_{s} \psi_{s}^{*}\left(\mathbf{r}^{\prime}\right) \psi_{s}(\mathbf{r}) e^{i \epsilon_{s}\left(t-t^{\prime}\right)}$. This quantity is actually a sort of one-hole Green's function, which will show up in the following in the more formal discussion of the problem.
} 


\section{GREEN'S-FUNCTION THEORY}

The Green's-function technique is indeed extremely useful: it allows one to tackle directly the problems of calculating excitation and ionization energies, groundstate energies, transition matrix elements, absorption coefficients, and dynamical polarizabilities, as well as elastic and inelastic electron cross sections. Furthermore, self-consistent perturbation theories can be formulated in terms of the Green's function. The technique is fully discussed in many textbooks and we refer the reader to those for details (Abrikosov et al., 1963; Hedin and Lundqvist, 1969; Fetter and Walecka, 1971; Landau and Lifschitz, 1980), but in the following we outline the equations needed in the discussion. For further reading about the subtleties of the mathematical concepts involved, see the review by Farid (1999a) where this many-body approach is developed in detail together with the approximations to Hedin's equations discussed below.

\section{A. The concept of Green's functions and the self-energy}

The one-electron Green's function $G$ is defined (at zero temperature) as an expectation value with respect to the many-electron ground state $|N\rangle$,

$$
G\left(x t, x^{\prime} t^{\prime}\right)=-i\left\langle N\left|T \psi(x t) \psi^{\dagger}\left(x^{\prime} t^{\prime}\right)\right| N\right\rangle,
$$

where $\psi(x t)$ is the field operator in the Heisenberg picture, $x$ stands for three space coordinates $(\mathbf{r})$ plus one spin coordinate $(\xi)$, and $T$ is the time-ordering operator. In this equation, $\psi^{\dagger}(x, t)|N\rangle$ represents an $(N+1)$-electron state in which an electron has been added to the system at point $\mathbf{r}$ and time $t$. When $t^{\prime}<t$, the many-body Green's function gives the probability amplitude to detect an electron at point $\mathbf{r}$ and time $t$ when an electron has been added to the system at point $\mathbf{r}^{\prime}$ and time $t^{\prime}$. When $t^{\prime}>t$, the Green's function describes the propagation of a many-body state in which one electron has been removed at point $\mathbf{r}$ and time $t$, that is, the propagation of a hole. ${ }^{11} G$ is closely related to fundamental properties like charge and spin density or the total energy, and derived spectra like photoemission or Compton scattering. Thus, for example, the charge density is obtained through

$$
\rho(\mathbf{r} t)=-i \lim _{\tau \rightarrow 0^{+}} \int G(x t, x t+\tau) d \xi .
$$

Similarly, the total electronic energy can be deduced through the Galitskii-Migdal formula (Galitskii and Migdal, 1958)

$$
E=\frac{1}{2} \int d x \lim _{t^{\prime} \rightarrow t^{+}}\left[\frac{\partial}{\partial t}-i h(x)\right] G\left(x, t, x^{\prime}, t^{\prime}\right)_{x^{\prime} \rightarrow x},
$$

\footnotetext{
${ }^{11}$ When time-reversal symmetry holds, the Green's function is symmetric with respect to the interchange of the spatial coordinates. This general statement is also true for the density matrix.
}

where $h(x)$ is the density-independent part of the effective Hamiltonian. By inserting a complete set of $(N$ $+1)$ - or $(N-1)$-particle states (depending on the time ordering) between the field operators in Eq. (3.1), one obtains the Lehmann representation of the Green's function in terms of the amplitudes,

$$
f_{s}(x)= \begin{cases}\langle N|\psi(x)| N+1, s\rangle, & \epsilon_{s}>\mu \\ \langle N-1, s|\psi(x)| N\rangle, & \epsilon_{s}<\mu\end{cases}
$$

and in terms of the real energies $\epsilon_{s}=E(N+1, s)$ $-E(N)($ or $[E(N)-E(N-1, s)])$ for $\epsilon_{s}>\mu(<\mu)$ :

$$
G\left(x, x^{\prime}, \omega\right)=\sum_{s} \frac{f_{s}(x) f_{s}^{*}\left(x^{\prime}\right)}{\omega-\left[\epsilon_{s}+i \eta \operatorname{sgn}\left(\mu-\epsilon_{s}\right)\right]},
$$

where the small imaginary part $\eta$ is needed for the convergence of the Fourier transform to frequency space. The poles of $G$ hence correspond to electron addition and removal energies. In particular, the so-called spectral function $A(\omega):=|(1 / \pi) \operatorname{Im} G(\omega)|$ becomes

$$
A\left(x, x^{\prime} ; \omega\right)=\sum_{s} f_{s}(x) f_{s}^{*}\left(x^{\prime}\right) \delta\left(\omega-\varepsilon_{s}\right) .
$$

In the noninteracting case, where $|N\rangle,|N+1\rangle$ and $\mid N$ $-1\rangle$ are simply Slater determinants, only states with $|N+1, s\rangle=c_{s}^{\dagger}|N\rangle$ ( $c^{\dagger}$ is a creation operator) lead to nonvanishing Lehmann amplitudes. These amplitudes and the Lehmann energies are then equal to the eigenfunctions and eigenvalues of the corresponding one-electron Hamiltonian, and the spectral function consists of a set of $\delta$ peaks at those eigenvalues. Hence each peak corresponds to a particle. Furthermore, it can be easily seen that the Green's function reduces to the time-dependent electron or hole density matrix introduced in Sec. II.C. When the electron-electron interaction is turned on it is no longer possible to write $|N-1\rangle$ or $|N+1\rangle$ simply in terms of pure one-electron Slater determinants. Instead, the many-body wave function is a linear combination of many of them. For example, the $(N+1)$-particle wave function can be expressed as $|N+1, s\rangle=\Sigma_{l m} a_{l m}^{s} c_{m}^{\dagger}\left|N_{l}\right\rangle$, where $\left|N_{l}\right\rangle$ is an excited state of the $N$-particle system. Hence there will now be more nonvanishing contributions to the spectral function (3.5). If these contributions, merged together, form a clearly identifiable main structure, which can be thought to derive from a $\delta$ peak when the interactions are switched off, one can still work in a particle-like picture, associating each peak with a "quasiparticle." 12 With respect to an independent-particle $\delta$ peak, for the quasiparticle one

\footnotetext{
${ }^{12}$ The concept of quasiparticles was introduced by Landau (Landau, 1957a, 1957b, 1959; Landau and Lifschitz, 1980) in the Fermi-liquid theory of ordinary metals as a one-to-one correspondence between low-energy excitations of a free Fermi gas and those of the interacting electron liquid. A quasiparticle can be considered as the combination of a real particle (electron or hole) and a cloud of virtual electron-hole pairs surrounding it. Through mutual interactions quasiparticles can decay into other quasiparticles, leading to a finite lifetime.
} 
finds a spreading of the oscillator strength. Therefore the broadening carries valuable information about many-body correlation effects in the interacting system.

Another important quantity for the analysis of the optical spectra of interacting electron systems is the twoparticle Green's function, defined as

$$
G\left(1,2,1^{\prime}, 2^{\prime}\right)=-\left\langle N\left|T \psi(1) \psi(2) \psi^{\dagger}\left(2^{\prime}\right) \psi^{\dagger}\left(1^{\prime}\right)\right| N\right\rangle,
$$

where space, time, and spin coordinates are indicated in abbreviated form by numbers, i.e., $1=\left(\mathbf{r}_{1}, t_{1}, \xi_{1}\right)$. This function provides information about processes involving two-particle transitions and their interaction through the time ordering of the four times appearing in the equation. For the purpose of this review, only a few specific time orderings need be considered, in particular for $t_{1}, t_{1}^{\prime}>t_{2}, t_{2}^{\prime}$

$$
G\left(1,2,1^{\prime}, 2^{\prime}\right)=-\sum_{s} X_{s}\left(1,1^{\prime}\right) \widetilde{X}_{s}\left(2,2^{\prime}\right)
$$

in terms of the hole-particle (Bethe-Salpeter) amplitudes $X_{s}\left(1,1^{\prime}\right)=\left\langle N\left|T \psi(1) \psi^{\dagger}\left(1^{\prime}\right)\right| N, s\right\rangle$ and $\widetilde{X}_{s}\left(1,1^{\prime}\right)$ $=\left\langle N, s\left|T \psi(1) \psi^{\dagger}\left(1^{\prime}\right)\right| N\right\rangle$. Also the case $t_{1}, t_{1}^{\prime}<t_{2}, t_{2}^{\prime}$ describes particle-hole amplitudes, whereas other time orderings will lead to particle-particle or hole-hole pairings. This point is relevant here because only for holeparticle pairings do the states belong to the $N$-particle system. Therefore one can define a specific hole-particle Green's function $G^{h p}$ considering only those two time orderings $\left(t_{1}, t_{1}^{\prime} \gtrless t_{2}, t_{2}^{\prime}\right)$ :

$$
G\left(1,2,1^{\prime}, 2^{\prime}\right)=G^{h p}\left(1,2,1^{\prime}, 2^{\prime}\right)+\text { other orderings. }
$$

The "other orderings" terms correspond to poles of the two-particle Green's function that differ from $\pm[E(N, s)-E(N)]$ and that are important for different types of spectroscopies, e.g., Auger in the case of $G^{h h}$ (Cini, 1977, 1979).

\section{Connection to spectroscopic measurements}

In the Introduction we sketched the main spectroscopic measurements that can be described by the approaches presented in this review. It is now useful to build a bridge between those qualitative considerations and the abstract mathematics of the previous subsection.

In fact, the building block for the description of any spectrum involving the interaction of radiation and matter is the probability $\mathcal{P}$ for an excitation from the initial state $\left|\Psi_{0}\right\rangle$ (typically the ground state) to a set of final states $\left|\Psi_{f}\right\rangle$. According to the measurement, the initial or final states contain an additional photon. This process is well described by Fermi's golden rule:

$$
\mathcal{P}=2 \pi \sum_{f}\left|\left\langle\Psi_{f}|\Delta| \Psi_{0}\right\rangle\right|^{2} \delta\left(E_{f}-E_{0}\right),
$$

where $\Delta$ describes the perturbation due to the photon field. For a one-photon process, neglecting quadratic terms in the vector potential $\mathcal{A}, \Delta=(e / 2 m c)(\mathcal{A} \mathbf{p}$ $+\mathbf{p} \mathcal{A})$. High photon fluences (multiphoton processes) are excluded here.

From the transition probability, one can construct the spectra. In the case of photoemission (see Fig. 1), one measures the photocurrent, which is proportional to the probability per unit time of emitting a photoelectron of momentum $\mathbf{k}$, when the target is irradiated with photons of frequency $\omega$. Hence one looks explicitly at the photoelectron. As a consequence, the simple approximations for photoemission decouple the photoelectron from the target, usually treating it as a free propagating electron state. One then finds that the photoemission spectrum carries directly accessible information about the target itself, namely, about its electronic structure, or to be precise, the energy of holes (or electrons, for inverse photoemission). The final simple relation between the photoemission spectrum and the electronic structure of the sample is based on the classical three-step model (see, for example, Almbladh and Hedin, 1983, and Kevan, 1992): the photoelectron is excited by the photon, loses energy on its way to the surface, and finally has to pass the surface to propagate (as a free particle) to the detector. Only the first step, the excitation of the photoelectron, is contained in the so-called intrinsic spectrum, whereas losses on the way out of the target are called extrinsic. ${ }^{13}$

Starting from the transition probability [Eq. (3.8)], one can write down the photoelectron current of photoemission, using the initial-state energy $E_{0}=E_{N}+\omega$, i.e., the sum of the $N$-particle ground-state energy and the photon energy. In the intrinsic approximation, the final state $|\mathbf{k} ; N-1, f\rangle$ is approximated by $c_{\mathbf{k}}^{\dagger}|N-1, f\rangle$ with energy $\epsilon_{\mathbf{k}}+E_{N-1, f}$, where $\mathbf{k}$ and $\epsilon_{\mathbf{k}}$ are the momentum and the energy of the photoelectron. The photoelectron current reads then (Almbladh and Hedin, 1983)

$$
\begin{aligned}
J_{\mathbf{k}}(\omega)= & \frac{k}{4 \pi^{2}} \sum_{f} \delta\left(\epsilon_{k}-\epsilon_{f}-\omega\right) \\
& \times\left|\left\langle N-1, f\left|c_{\mathbf{k}} \sum_{i j} \Delta_{i j} c_{i}^{\dagger} c_{j}\right| N\right\rangle\right|^{2} .
\end{aligned}
$$

Here $\epsilon_{f}=E_{N}-E_{N-1, f}$, and $\Sigma_{i j} \Delta_{i j} c_{i}^{\dagger} c_{j}$ is the photon operator in second quantization. Using the argument that the high-energy one-electron state $\mathbf{k}$ does not change the state of the $(N-1)$-electron system, and that it makes a negligible contribution to the virtual one-electron excitations contained in the ground state $N$ (that is $c_{\mathbf{k}}|N\rangle$ $=0$ ), one can write the photoelectron current as

$$
J_{\mathbf{k}}(\omega)=\frac{k}{4 \pi^{2}} \sum_{i j} \Delta_{k i} A_{i j}\left(\epsilon_{k}-\omega\right) \Delta_{j k},
$$

where $A_{i j}$ are the matrix elements of the spectral function (3.5) between one-electron orbitals,

$$
A_{i j}\left(\epsilon_{k}-\omega\right)=\int d x d x^{\prime} \psi_{i}^{*}(x) \psi_{j}\left(x^{\prime}\right) A\left(x, x^{\prime}, \epsilon_{k}-\omega\right) .
$$

\footnotetext{
${ }^{13}$ For high-energy photoelectrons this contribution is, in general, much smaller than the intrinsic part.
} 
If one further assumes that the diagonal elements of $A$ are dominating (which is exact for independent electrons) and that the $\Delta$ are constant, the photoemission spectrum is proportional to the trace of the interacting spectral function (also neglecting electron-phonon coupling and impurity effects).

From Eq. (3.5) one can see that the spectral function has a direct link to the density of available states for the addition and removal of electrons. In fact, for independent particles the trace of the spectral function is proportional to the density of occupied and empty states of the system, respectively, and provides a good approximation for the direct and inverse photoemission spectra. As we have seen, the same is true for the interacting electron case: the trace of $A\left(x, x^{\prime} ; \omega\right)$ can be accessed experimentally. In this way, the quasiparticle spectrum is directly measured in experiments such as direct, inverse and time-resolved two-photon photoemission (see Fig. 1 for a schematic diagram of the first two). Still, final-state, extrinsic, and surface (transport) effects, neglected in this discussion, can play an important role in some specific experimental configurations.

The absorption coefficient, by contrast, is defined as the ratio of the energy removed from the incident beam per unit time and per unit volume to the incident flux. This energy is absorbed thanks to transitions from the initial to any final state, with the sole constraint of energy conservation. There is, in general, no simple assumption concerning the final state as there is in photoemission, and one has to deal with holes and electrons at the same time. It would not be a good approximation for the final state to decouple the excited electron from the others, nor to describe it by a scattering (plane-wave) state. One has therefore to sum over all possible $N$-particle excited states in order to obtain the absorption coefficient. The energy differences appearing in the $\delta$ function are now $E_{N, f}-\left(E_{N}+\omega\right)$, and the sum over all states leads to a density-density correlation function (or density response function). ${ }^{14}$ The latter can be obtained from the time-ordered (or retarded) two-particle Green's function $G^{h p}\left(1,2,1^{\prime}, 2^{\prime}\right)$ [Eq. (3.7)], by contracting some of its arguments and by taking suitable averages or integrals of the resulting (microscopically varying) functions. That is, the microscopic polarizability of Eq. (1.3) or the density response function entering in Eqs. (2.2) and (2.5) are obtained from a $G^{h p}$ where the limit $\left(1^{\prime}\right) \rightarrow\left(1^{+}\right) ;\left(2^{\prime}\right) \rightarrow\left(2^{+}\right)$has been taken. In the

\footnotetext{
${ }^{14}$ Indeed, using the standard Kubo formula for the densitydensity response function (Abrikosov et al., 1963) $\chi\left(x, t ; x^{\prime}, 0\right)$ $=-i \theta(t)\left\langle N\left|\left[\rho(x, t), \rho\left(x^{\prime}, 0\right)\right]\right| N\right\rangle$, together with the closure relation of $N$-particle states $|N, f\rangle$ and the time evolution of the density operator $\rho(x, t)=e^{i H_{0} t} \rho(x, 0) e^{-i H_{0} t}$, one finds the response function $\chi\left(x, t ; x^{\prime}, 0\right)$ $=-i \theta(t)\left[\Sigma_{f} B_{f}(x) B_{f}^{*}\left(x^{\prime}\right) e^{i\left(E_{N}-E_{N, f}\right) t}-\right.$ c.c. $]$, where $B_{f}(x)$ $=\langle N|\rho(x, t)| N, f\rangle$ and $E_{N, f}$ is the energy of the $f$ th $N$-particle excited state. Then its time Fourier transform has poles at the true excitation energies of the $N$-particle system, $\omega= \pm\left(E_{N}\right.$ $\left.-E_{N, f}\right)$.
}

RPA approximation, $P_{I Q P}$ is derived by taking $G^{h p}$ as the product of two one-particle Green's functions: $G\left(1,2,1^{\prime}, 2^{\prime}\right)=G\left(1,2^{\prime}\right) G\left(2,1^{\prime}\right)$, which becomes $G(1,2) G(2,1)$ after the contraction. More details are given in Sec. IV.B.2.

\section{Dyson's equation: the self-energy operator $\Sigma$}

The full one-particle Green's function cannot be computed exactly, and one therefore needs a method to derive a physically sound approximation. In the simple case of the Hartree-Fock approximation, $G$ is written directly in terms of the Hartree-Fock eigenvalues and eigenfunctions as $G^{H F}\left(x, x^{\prime}, \omega\right)=\Sigma_{s} \psi_{s}(x) \psi_{s}^{*}\left(x^{\prime}\right) /\{\omega$ $\left.-\left[\epsilon_{s}+i \eta \operatorname{sgn}\left(\mu-\epsilon_{s}\right)\right]\right\}$. The inverse of $G^{H F}$ is hence linked to the Hartree-Fock Hamiltonian $H^{H F}$ :

$$
\left(G^{H F}\right)^{-1}\left(x, x^{\prime}, \omega\right)=\omega-H^{H F}\left(x, x^{\prime}\right) .
$$

For the general case, it is therefore reasonable to write the frequency Fourier transform of $G^{-1}$ as

$$
G^{-1}\left(x, x^{\prime}, \omega\right)=\left\{\delta\left(x-x^{\prime}\right)\left[\omega-H_{H}(x)\right]-\Sigma\left(x, x^{\prime}, \omega\right)\right\},
$$

where $H_{H}$ contains the kinetic-energy operator, the external and the Hartree potential, and $\Sigma$ is supposed to include the Fock exchange and the rest of the Coulomb interaction (correlation). This means that in this Dyson equation for $G$ the exchange (the Fock term) and correlation contributions are included in an operator that is in principle unknown, the self-energy $\Sigma .{ }^{15}$ Since the right-hand side of Eq. (3.13) contains $\omega$, i.e., the Fourier transform of a time derivative, implicit expressions for $\Sigma$ can be found through an evaluation of the equation of motion for the Green's function. This scheme gives rise to a set of coupled differential equations in which higher-order Green's functions appear. Those equations can be exactly decoupled in only a few cases, and one has to resort to approximations to get a practical, solvable scheme. However one can sum up the series of coupled equations for the Green's functions in a perturbative way and recast all the information in the operator $\Sigma$, as we shall see later.

Suppose for the moment that $\Sigma$ is known. Expressing $G$ in terms of the Lehmann amplitudes and energies [Eq. (3.3)], one finds, after taking (for a discrete level) the $\omega \rightarrow \varepsilon_{s}$ limit,

$\int\left\{\delta\left(x-x^{\prime \prime}\right)\left[\varepsilon_{s}-H_{H}(x)\right]-\Sigma\left(x, x^{\prime \prime}, \varepsilon_{s}\right)\right\} f_{s}\left(x^{\prime \prime}\right) d x^{\prime \prime}=0$,

i.e., the Dyson equation for the quasiparticle energies $\varepsilon_{s}$ and amplitudes $f_{s}$. The amplitudes $f_{s}$ form a complete set but are nonorthogonal, since the self-energy operator is energy dependent. This also implies that the equation can have more solutions than in the static case. In-

\footnotetext{
${ }^{15}$ See Farid (2001) for a detailed discussion of the general properties of the dynamical self-energy operator, including an analysis of the shortcomings of the usually applied approaches.
} 
stead, if the electron-electron interaction is smoothly switched off, those amplitudes tend to form the standard orthonormal set of an independent-particle system. This leads again to the situation described above, with the difference between the isolated independent-particle peaks and the broad quasiparticle ones containing a certain number of nonvanishing Lehmann amplitudes. Dyson's equation (3.14) indeed has a one-electron form (single-particle Schrödinger-like) but the operator $\Sigma$ has very nontrivial correlation effects built in and is far from a mean-field approximation.

In order to deal more easily with the pole structures of $G, \Sigma$, etc., it is convenient to generalize all quantities from the real frequency axis $\omega$ to the complex plane $z$, i.e., to make an analytic continuation. The one-particle Green's function is then written in terms of left and right solutions

$$
G(z)=\sum_{s} \frac{\phi_{s}^{r}(z) \phi_{s}^{l^{*}}(z)}{z-E_{s}(z)}
$$

defined by the general Dyson equation to be solved in the full complex plane,

$$
E_{s}(z)=z
$$

where

$$
\begin{aligned}
& {\left[H_{0}+\Sigma(z)\right] \phi_{s}^{r}(z)=E_{s}(z) \phi_{s}^{r}(z),} \\
& {\left[H_{0}+\Sigma(z)\right]^{\dagger} \phi_{s}^{l *}(z)=E_{s}^{*}(z) \phi_{s}^{l *}(z) .}
\end{aligned}
$$

The self-energy $\Sigma$ here plays the role of an effective potential for an electron or hole added to the system which is, in general, complex, nonlocal, and energy dependent. This potential arises from the exchange and from the response of the rest of the electrons to the presence of the additional particle. $\Sigma(\omega)$ is real when $\omega$ is set to an energy value below the first inelastic threshold of the system.

Thus one has achieved an exact one-particle picture at the price of introducing a complicated effective potential. The non-hermiticity and energy dependence of $\Sigma$ imply that the quasiparticle wave functions are energy dependent and not necessarily orthogonal; however, both the Green's function and Dyson's equations admit a biorthogonal spectral representation (in terms of left and right eigenfunctions). ${ }^{16}$ The important point to note is that now one is dealing with the energies $E_{s}(z)$, which are in principle complex.

The mathematical details of the analytic continuation are nontrivial and have important consequences. For a rigorous and complete treatment we refer the reader to the review paper of Farid (1999a). It is, however, useful to illustrate the connection between the Lehmann representation and the analytic continuation by an example:

\footnotetext{
${ }^{16}$ The biorthonormal and Lehmann representations are both exact. Even if they look similar they are not identical, since in the former case one works with the analytic continuations (Farid, 1999a).
}

Suppose that some matrix element of a Green's function is given in the Lehmann representation by

$$
G(\omega)=\sum_{s} \frac{g(s)}{\omega-\epsilon_{s}+i \eta} .
$$

For simplicity, let us consider a case in which $g(s)$ $=(1 / \pi) E_{2} /\left[\left(s-E_{1}\right)^{2}+E_{2}^{2}\right]$ and $\epsilon_{s}=s$. Given this particular form of $g(s)$, taking the thermodynamic limit (i.e., making $s$ a continuous variable) yields a spectral function $A(\omega)=1 / \pi|\operatorname{Im}[G(\omega)]|$ with a Lorentzian form:

$$
A(\omega)=\frac{1}{\pi} \frac{E_{2}}{\left(\omega-E_{1}\right)^{2}+E_{2}^{2}} .
$$

On the other hand, we can also write

$$
A(\omega)=\frac{1}{\pi}\left|\operatorname{Im}\left(\frac{1}{\omega-\left(E_{1}+i E_{2}\right)}\right)\right| ;
$$

thus the Lorentzian, which in the beginning was given by the imaginary part of a function containing a series of infinitely close-lying poles on the real axis (a branchcut), in this particular case can also be described by the imaginary part of another function, defined in the whole complex plane, having a single, but complex, pole $\left(E_{1}\right.$ $\left.+i E_{2}\right)$. The position of the peak of $A(\omega)$ is given by the real part $E_{1}$, and the imaginary part $E_{2}$ gives its width. This simple example demonstrates the connection between the Lehmann and the complex-pole representation of the Green's function, in the case of a single quasiparticle pole. It also explains why the complex representation is advantageous-in this example it replaces the search for a branchcut by the search for one complex pole. Moreover, it illustrates the meaning of $E_{1}$ and $E_{2}$ : the real part of the quasiparticle energies solution of Dyson's equation gives the band structure, and the imaginary part the quasiparticle damping (electron dynamics). The solution can be real valued only for excitations with infinite lifetime $\left[\operatorname{Im} \Sigma\left(z=\varepsilon_{s}\right)=0\right.$; the discrete part of the spectrum]. However, for energy ranges where $\varepsilon$ forms a continuum, $\Sigma\left(x, x^{\prime} ; \varepsilon\right)$ is also complex and Dyson's equation still has a solution with a complex $z$. In principle, this non-Hermitian eigenvalue problem can be solved for each and every value of $z$ in the complex plane (with the corresponding set of eigenvalues and eigenvectors). Finally, this simple example is meant to explain why the existence of complex quasiparticle energies is not in contradiction with the fact that $\varepsilon_{s}$ appearing in the Lehmann representation of the Green's function are always real quantities.

In order to extend the quasiparticle picture, one can return to the description of a photoelectron spectrum in terms of the Green's function and self-energy operator (in its analytic continuation, i.e., using complex energies), by considering the matrix elements of Eq. (3.15):

$$
G_{i i}(\omega)=\frac{1}{\omega-E_{i}(\omega)} .
$$

If one expands this expression for energies $\omega$ close to the generally complex quasiparticle energy $E_{i}$, one obtains

$$
G_{i i}(\omega) \approx \frac{Z_{i}}{\omega-E_{i}},
$$


where $Z_{i}$ is the complex renormalization factor,

$$
Z_{i}=\frac{1}{1-\left.\frac{\partial \Sigma_{i i}(\omega)}{\partial \omega}\right|_{\omega=E_{i}}} .
$$

The $i$ th matrix element of the spectral function for $\omega$ close to $E_{i}$ then reads

$$
A_{i i}(\omega)=\frac{1}{\pi} \frac{\left|\operatorname{Im} E_{i} \operatorname{Re} Z_{i}-\left(\omega-\operatorname{Re} E_{i}\right) \operatorname{Im} Z_{i}\right|}{\left(\omega-\operatorname{Re} E_{i}\right)^{2}+\left(\operatorname{Im} E_{i}\right)^{2}} .
$$

If $\left|\operatorname{Im} E_{i}\right|$ is small, $A_{i i}(\omega)$ has a sharp Fano peak at $\omega$ $=\operatorname{Re} E_{i}$ of width $\Gamma_{i}=\left|\operatorname{Im} E_{i}\right|$ and strength $\left|\operatorname{Re} Z_{i}\right|$, with the asymmetry set by $\operatorname{Im} Z_{i}$. $Z_{i}$ is hence a measure of the strength of the quasiparticle pole and determines the specific shape of the corresponding excitation in the spectral function. Even for weakly correlated systems like $s p$ metals and valence semiconductors $\left|\operatorname{Re} Z_{i}\right|$ is far from 1 and can typically be in the range 0.6-0.9 (Hedin and Lundqvist, 1969; Hybertsen and Louie, 1986; Godby et al., 1988). The sum of all resonances, i.e., the trace of $A$, will finally determine the specific shape of the photoelectron spectrum.

\section{B. Hedin's equations}

As discussed in the Introduction (Sec. I.B), when going beyond Hartree-Fock one has to deal with electron relaxation and correlation effects, which can also be described as dynamical screening effects. In fact, one can write an expansion of the self-energy in terms of a screened Coulomb potential $W$, by demanding that the total energy be stationary with respect to variations of the Green's function (Hedin, 1965). In this process the self-energy should be seen as a functional of the Green's function $(\Sigma[G])$. The use of $W$ instead of the bare Coulomb potential is physically more sound: conventional many-body perturbation theory suffers in fact from various convergence problems, which have to be bypassed with care (see, e.g., Farid, 1999b).

In fact, interactions in a real many-body system are screened to a large extent, and therefore $W$ should be a much better behaved quantity for the development of a perturbation expansion than the bare Coulomb potential. The latter is known to lead to convergence problems in the range of densities of normal metals and semiconductors (Hedin, 1965, 1999; Hedin and Lundqvist, 1969, 1971). This expansion allows one to write the many-body problem as a closed set of five integral equations introduced by Hedin (1965), which relate the Green's function, self-energy, polarization propagator, and vertex function. The equations can be obtained by introducing a local time-dependent source term in the Hamiltonian which directly couples to the particle density. This new source term is set to zero once the final equations are obtained. In this way Hedin derived a selfconsistent scheme written in terms of the following set of coupled equations [here, as before, $1=\left(\mathbf{r}_{1}, t_{1}, \xi_{1}\right)$, and $v$ stands for the bare Coulomb interaction]:

$$
\begin{aligned}
\Sigma(12)= & i \int G(13) \Gamma(324) W(41) d(34) \\
W(12)= & v(12)+\int v(13) P(34) W(42) d(34) \\
P(12)= & -i \int G(13) G(41) \Gamma(342) d(34) \\
\Gamma(123)= & \delta(12) \delta(13) \\
& +\int \frac{\delta \Sigma(12)}{\delta G(45)} G(46) G(75) \Gamma(673) d(4567)
\end{aligned}
$$

where $P(12)$ is the time-ordered polarization operator, $W(12)$ is the dynamical screened interaction, and $\Gamma(123)$ is the vertex function. The fifth equation is the Dyson equation, which links $G$ and $\Sigma$ [see Eq. (3.13)]. This way of writing the equations is in fact appealing, since it highlights the important physical ingredients: the polarization [Eq. (3.24)], which contains the response of the system to the additional particle or hole, is built up by the creation of pairs of particles and holes (the two Green's functions). The vertex function $\Gamma$ contains the information that the hole and the electron interact. $\Gamma$, in turn, is determined by the change in the potential upon excitation [Eq. (3.25)].

\section{The iterative approach}

Hedin's equations together with Dyson's equation form a set of equations that must in principle be solved self-consistently for $G$. This means that the Green's function used to calculate the self-energy should coincide with the Green's function obtained from the Dyson equation with the very same self-energy. It is obvious that this is a very difficult task, and that one must in practice find a simplification of Hedin's equations. Since it is not possible to find a straightforward, well-defined and convergent perturbation expansion in some small parameter, the approach to simplifying the equations is somewhat arbitrary and needs to be analyzed in detail. A self-consistent calculation of the interaction of lowenergy electrons with an electron gas was first carried out by Quinn and Ferrell (1958). They performed a selfenergy calculation of electron-electron scattering rates near the Fermi surface and derived a formula for the inelastic lifetime of hot electrons which is exact in the high-density limit. These free-electron-gas calculations were extended by Ritchie (1959) ${ }^{17}$ and Quinn (1962) to include, within the first Born and random-phase approximations, energies away from the Fermi surface, and by Adler (1963) and Quinn (1963) to take into account the effects of the presence of a periodic lattice and, in particular, the effect of virtual interband transi-

\footnotetext{
${ }^{17}$ The $1 / 2$ factor in front of $z^{2}$ in the expansion of $f_{1}$ just before Eq. (6.15) of this reference must be replaced by $1 / 3$, as done in a subsequent paper (Ritchie and Ashley, 1965).
} 
tions (Quinn, 1963). In their original work, Quinn and Ferrell (1958) had already given the $G W$ expression for $\Sigma$, but did not recognize its usefulness beyond the case of an electron gas and only evaluated the results as a density $\left(r_{s}\right)$ expansion. Further studies for quasiparticle excitations and lifetimes in a homogeneous electron gas were done by Hedin (1965) and Lundqvist $(1967,1968)$.

\section{First iteration step: the $G W$ approximation}

The simplest approximation to $\Gamma(123)$ assumes this operator to be diagonal in space and time coordinates, $\Gamma(123)=\delta(12) \delta(13)$ : the so-called vertex corrections are neglected. This implies that the irreducible polarizability is given by noninteracting quasielectronquasihole pairs,

$$
P(12)=-i G(12) G\left(21^{+}\right) .
$$

It can be evaluated explicitly by using Eq. (3.4). After a Fourier transformation, and taking into account the small imaginary parts of the energies, one obtains the formula (1.4) in its time-ordered version; this approximation is in fact again the RPA form.

For the self-energy, this approximation yields the form

$$
\Sigma(12)=i G(13) W(31) \text {, }
$$

the so-called $G W$ approximation as introduced by Hedin (1965). Of course, the Dyson equation for the Green's function $\mathbf{G}=G_{0}+G_{0} \Sigma G$ still has to be added, and even at the $G W$ level one has to deal with a manybody self-consistent problem. However, the $G W$ approximation is a comparatively simple expression for the self-energy operator, which in principle allows the Green's function of an interacting many-electron system to be computed by starting from the Green's function $G_{0}$ of a hypothetical independent-particle system with an effective one-electron potential, and iterate to selfconsistency. The $G W$ extends the well-known HartreeFock approximation, in which the self-energy is the exchange potential $\Sigma_{x}$, by replacing the bare Coulomb potential $v$ by the dynamically screened potential $W$, e.g., $\Sigma_{x}=i G v$ is replaced by $\Sigma_{G W}=i G W$. The selfenergy operator now consists of a dynamically screened exchange potential plus a dynamical Coulomb hole. In fact, the static approximation of the $G W$ self-energy is none other than the COHSEX formula [Eq. (1.2)] discussed in the introduction. $G W$ has been shown to be physically well motivated and to be superior to the Hartree-Fock approximation, especially for metals, where Hartree-Fock (i.e., using the bare Coulomb potential) leads to unphysical results (Hedin and Lundqvist, 1969). The dynamically screened interaction $W$ introduces energy-dependent correlation effects absent in the one-particle picture. Further, an energy-dependent correlation decreases the Hartree-Fock band gap by raising the valence-band energy and lowering the conduction-band energy. There is some empirical evidence that supports the idea that even in the first iteration (that is, using just the noninteracting Green's function $G_{0}$ ) one obtains quite accurate results for one- electron properties such as the excitation energy (Hybertsen and Louie, 1985, 1986; Godby et al., 1986, 1987, 1988; Aryasetiawan and Gunnarsson, 1998) and the quasiparticle lifetime (Campillo et al., 1999; Schöne et al., 1999; Campillo, Pitarke, et al., 2000; Echenique et al., 2000; Campillo, Rubio, et al., 2000; Campillo, Silkin, et al., 2000; Keyling et al., 2000; Silkin et al., 2001; Spataru et al., 2001). This is important for practical applications of the $G W$ approach since, despite its formal simplicity, the practical solution of the self-consistent $G W$ equations is a formidable task, which has been carried out only recently: self-consistent calculations were performed for the homogeneous electron gas (Holm and von Barth, 1998; Holm and Aryasetiawan, 2000; GarcíaGonzález and Godby, 2001), simple semiconductors, and metals (Shirley, 1996; Schöne and Eguiluz, 1998). Selfconsistency modifies the one-electron excitation spectrum (excitation energies and lifetimes) as well as the calculated screening properties. The results turn out to be worse than those of the nonself-consistent $G_{0} W_{0}$ calculations ${ }^{18}$ or those obtained within the so-called $G W_{0}$, in which only the explicit $G$, and not $W$, is updated at every iteration, thus achieving partial selfconsistency in $G$ (von Barth and Holm, 1996). Improvements have been obtained, in particular concerning the position of plasmon satellites, using a partially selfconsistent $G$, i.e. wave functions of order zero but updated quasiparticle energies (Bechstedt et al., 1994). The problem of self-consistency in $G W$ calculations has been investigated more deeply in such simple systems as the homogeneous electron gas or an exactly solvable Hubbard model. The main outcome of the self-consistent $G W$ calculation for the homogeneous electron gas (Holm and von Barth, 1998; Holm and Aryasetiawan, 2000; García-González and Godby, 2001) is that the total energy computed with the Galitskii and Migdal (1958) formula turns out to be strikingly close to the total energy calculated using quantum Monte Carlo (Ceperley and Alder, 1980; Ortiz et al., 1999). Here few sum rules already determine most of the energy contributions in the homogeneous electron gas. Encouraging results are also obtained for the electron gas in three and two dimensions, even for those ranges of densities for which the $G W$ approach is often supposed to fail (GarcíaGonzález and Godby, 2001) and for inhomogeneous systems such as bulk, surfaces, and interfaces (SánchezFriera and Godby, 2000; García-González and Godby, 2002), and dimers (Aryasetiawan et al., 2002b). This result may be related to the fact that the self-consistent $G W$ scheme conserves electron number, energy, and total momentum (that is, it fulfills the microscopic conservation laws; Martin and Schwinger, 1959, Baym and Kadanoff, 1961; Baym, 1962). However, concerning spectroscopic properties a systematic deterioration in the description of the bandwidth, quasiparticle excita-

\footnotetext{
${ }^{18}$ In this scheme $G_{0} W_{0}$, the full $G$ has been replaced by $G_{0}$ and the corresponding screened Coulomb potential is called $W_{0}$.
} 
tion and lifetimes, and plasmon satellites is found. Results along the same lines were obtained in the case of an analytically solvable finite Hubbard cluster recently used to compare the performances of several types of GW calculations (Verdozzi et al., 1995; Pollehn et al. 1998; Schindlmayr et al., 1998).

An important conclusion of this subsection is that, if one is interested in the excitation spectrum, selfconsistent $G W$ performs worse than the simpler $G_{0} W_{0}$ scheme; efforts should be directed towards consistently including vertex corrections in the calculations (see next section).

\section{Second iteration step: vertex correction in $P$ and $\Sigma$}

Having arrived at the $G W$ expression for the selfenergy (or any approximation for $\Sigma$ other than $\Sigma=0$ ), one can go again through Eq. (3.22)-(3.25), starting with the latter. Now, for a nonvanishing functional derivative $\delta \Sigma / \delta G$, one obtains a correction to the bare vertex $\Gamma$ $=\delta$; this is the linear response of the self-energy to a change in the total potential of the system. This correction affects the polarizability in Eq. (3.24) - the two Green's functions in $P$ are no longer decoupled. In fact, vertex corrections account for exchange-correlation effects between an electron and the other electrons in the screening density cloud. In particular this includes the electron-hole attraction (excitonic effects) in the dielectric response, which we shall look at later.

The improved $\Gamma$ and $P$ can then be used, through Eqs. (3.23) and (3.22), in order to construct a new self-energy. This iteration step is still important, since, for example, long-range vertex corrections are needed in order to improve the structure of plasmon satellites (Aryasetiawan and Gunnarsson, 1998). Also the fact that selfconsistency causes the results of GW spectroscopy calculations to deteriorate suggests that vertex corrections are important (Verdozzi et al., 1995). On the other hand, as noted by DuBois (1959), vertex corrections enhance correlation functions as the density-density response, whereas the inclusion of self-energy effects in the Green's function leads to a reduction. Similar conclusions were reached by Hong and Mahan (1994). Therefore one faces competing effects between selfconsistency and vertex corrections: contributions from vertex corrections and self-consistency tend to cancel to a large extent for the 3D homogeneous electron gas. However, vertex corrections in quasi-2D electron-gas systems (like narrow quantum wells) are more important for the description of the quasiparticle band-gap renormalization and lifetimes (Marmorkos and Das Sarma, 1991). ${ }^{19}$ The cancellation of vertex corrections with self-consistency seems to be a quite general feature. Vertex correction diagrams have also been found by de

\footnotetext{
${ }^{19}$ For the case of the semimetallic graphite (quasi-2D system) it has been shown by Spataru et al. (2001) that $G_{0} W_{0}$ describes the observed energy dependence of the electron lifetimes but not the absolute value.
}

Groot et al. (1996) to partially cancel the selfconsistency effects in the case of a quasi-onedimensional semiconducting wire. Their results show that, after systematic inclusion of all lowest-order corrections both to the RPA polarizability and to the $G W$ self-energy, the band gap is roughly the same as at the first iteration $G_{0} W_{0}$. Cancellation of the first-order vertex and self-consistency corrections, however, has been found only to a minor extent for the band gaps of bulk silicon and diamond (Ummels et al., 1998). The authors have included vertex and self-consistency corrections to first order and found that corrections to the polarizability largely compensate each other, while this is not true for the gaps which become larger than the $G W$ ones by 0.7 and $0.4 \mathrm{eV}$ for diamond and silicon, respectively. ${ }^{20}$

There are also cancellations between the same vertex correction used in different places; in fact, Mahan and Sernelius (1989) emphasize that a consistent way of handling vertex corrections is to include identical vertex corrections in both the polarization and the self-energy operator.

The simplest improvement to the $G W$ approximation in Eq. (3.22) consists in introducing a vertex correction consistent with the starting LDA calculation of the oneelectron orbitals. The DFT exchange-correlation potential is regarded as an approximation to the self-energy (Del Sole et al., 1994). Based on this idea, the vertex $\Gamma$ can be easily expressed in terms of the functional derivative $\delta V_{x c} / \delta \rho$. This so-called $G W \Gamma$ approximation (Rice, 1965; Hybertsen and Louie, 1986; Mahan and Sernelius, 1989; Del Sole et al., 1994; Mahan, 1994) reads in compact notation

$$
\begin{aligned}
& \Gamma=\frac{1}{1-f_{x c} P_{0}}, \\
& W=W_{1} \equiv \frac{\left(1-f_{x c} P_{0}\right) v}{1-\left(v+f_{x c}\right) P_{0}}, \quad \mathbf{G}=\mathbf{G}_{0}
\end{aligned}
$$

and thus

$$
\Sigma=i G_{0} W_{1} \Gamma=i G_{0} \frac{v}{1-\left(v+f_{x c}\right) P_{0}} .
$$

$\Sigma$ is hence again of the $G W$ form, but with an effective screening $W_{2}:=v /\left[1-\left(v+f_{x c}\right) P_{0}\right]$ which is in fact the testcharge electron dielectric function of linear-response theory (Del Sole et al., 1994; Hedin, 1999). The corrections given by $f_{x c}$ hence account for exchangecorrelation effects at two levels. The first level concerns the electrons of the "screening medium," with an exchange-correlation hole around the electrons; when an electron is participating in the dielectric screening

\footnotetext{
${ }^{20}$ Note that there are also cancellations in the $T$-matrix approximation where short-range interactions between two localized holes (electrons) can be described by a series of ladder diagrams exactly representing all repeated binary collisions (Kanamori, 1963). Due to the effects of the vertex corrections, the self-consistent version of the $T$ matrix (using dressed propagators in the ladder) is found to be inferior to the nonself-consistent one (Cini and Verdozzi, 1986).
} 
others are less likely to be found nearby. Second, the potential induced by the additional electron or hole also includes exchange-correlation interactions between the particle and the system electrons, and not only the classical Coulomb interaction (since the particles are in fact indistinguishable). The vertex correction has to be put in both $P$ and $\Sigma$ in order to obtain this result (a vertex correction in $P$ alone yields the testcharge-testcharge dielectric function). Results for silicon (Del Sole et al., 1994) show that the quasiparticle energy gaps obtained using the RPA screening (as is done in standard $G W$ calculations) or $W_{2}$ are close, whereas using $\Sigma$ $=i G_{0} W_{1}$, i.e., putting the vertex correction only in $P$, alters the results, which gives evidence for the importance of consistent corrections. Support for this sort of $G W \Gamma$ approximation can already be found in the work of Rice (1965). He showed that, using Hubbard's energy expression corrected for exchange effects in an electron gas, a functional derivative of the total energy with respect to the electron occupation number gave a quasiparticle energy corresponding to $\Sigma=i G_{0} W_{2}$.

The main drawback of the LDA-based $G W \Gamma$ approximation is the fact that results for bandgaps in semiconductors as well as valence bandwidths are very close to the standard $G W$ values. Further improvements might be obtained by using better approximations to the TDDFT kernel, including nonlocality and memory effects (see Sec. V.C), or it might be necessary to work with the true nonlocality of the exchange-correlation self-energy, which means using a four-point kernel (see Sec. II.C). An example of such a calculation has been given by Shirley and Martin (1993), who performed $G W$ calculations for atoms starting from Hartree-Fock and using a vertex correction consistent with the Fock exchange term. Shirley and Martin found that the results for Group I, II, IV, and VIII elements are similar to those of ordinary $G W$ without vertex corrections, again suggesting large cancellations. It should, however, be noted that the choice of the "reference" state was crucial. For example, electron removal energies from the neutral state were calculated as electron addition energies to the corresponding singly ionized state. This might be seen as a partial inclusion of important vertex and self-consistency corrections already in the reference state, for calculations both with and without explicit vertex corrections.

Systematic vertex corrections can hence be obtained through an iterative solution of Hedin's equations. One can push this scheme further by using the $G W$ approximation for $\Sigma$ in the equation for the vertex function, and so on. An explicit formula for the vertex function corresponding to the full second cycle of iteration can be obtained which mixes certain diagrams of different orders in the screened interaction. Schindlmayr and Godby (1998) have shown that the vertex to order $(n+1)$,

$$
\begin{aligned}
\Gamma^{(n+1)}(123)= & \delta(12) \delta(13)+\int d(4567) \frac{\delta \Sigma^{(n)}(12)}{\delta G^{(n)}(45)} \\
& \times G^{(n)}(46) G^{(n)}(75) \Gamma^{(n+1)}(673),
\end{aligned}
$$

can be expressed in terms of the lowest-order equivalents of all quantities except $\Sigma^{(n)}$ :

$$
\begin{aligned}
\Gamma^{(n+1)}(123)= & \delta(12) \delta(13)+\int d(4567) \frac{\delta \Sigma^{(n)}(12)}{\delta G^{(0)}(45)} \\
& \times G^{(0)}(46) G^{(0)}(75) \Gamma^{(1)}(673) .
\end{aligned}
$$

The appearance of the lowest order reduces the numerical effort substantially. In particular $G^{(0)}$ can be chosen such that it contains no satellite spectrum, but only a set of robust quasiparticle excitations. In the self-consistent limit $n \rightarrow \infty$ the previous equation implies a relation between the exact self-energy and vertex function. $\mathrm{Nu}-$ merical results for a model of strongly correlated electrons have indicated that this method yields improved excitation energies, in particular concerning the satellite spectrum. Nevertheless, to our knowledge this approach has never been applied to real systems. Instead, a slightly different version of the first iteration step is widely used, namely, the expression

$$
\begin{aligned}
\Gamma^{(n+1)}(123)= & \delta(12) \delta(13)+\int d(4567) \frac{\delta \Sigma^{(n)}(12)}{\delta G^{(n-1)}(45)} \\
& \times G^{(n)}(46) G^{(n)}(75) \Gamma^{(n+1)}(673)
\end{aligned}
$$

in its lowest-order version,

$$
\begin{aligned}
\Gamma^{(2)}(123)= & \delta(12) \delta(13)+\int d(4567) \frac{\delta \Sigma^{(1)}(12)}{\delta G^{(0)}(45)} \\
& \times G^{(1)}(46) G^{(1)}(75) \Gamma^{(2)}(673) .
\end{aligned}
$$

Note that the only difference between Eqs. (3.30) and (3.32) is the version of the Green's function with respect to which the functional derivative of $\Sigma$ is taken. The consequences might, however, be important, because whereas it is obvious that already the first-iteration solution of the integral equation (3.33) will shift the poles of $P=-i G G \Gamma$, this is not obvious in the case of Eq. (3.31). Rather, for $n=1$ Eq. (3.31) multiplied by $-i G^{(1)} G^{(1)}$ yields a first-order polarizability with poles coming from the poles of both $G^{(0)}$ and $G^{(1)}$.

In fact, Eq. (3.33) is the starting point for the derivation of the Bethe-Salpeter equation, which correctly yields features like bound excitons in the absorption spectra, as we shall show in Sec. IV.B.3.

Obviously, choices like the one between Eqs. (3.30) and (3.32) are crucial only because one typically does not want to iterate to infinity, but to find the "best" firstiteration step, which is not well defined. This kind of problem regularly shows up when one is dealing with many-body Coulomb equations.

Another way of partially summing higher-order diagrams for describing vertex corrections to the valence electron Green's function is given by an exponential expression very similar to the (almost exact) solution obtained for the core electron Green's function, as first shown by Hedin (1980). This approximation can also be derived as the first term in a cumulant expansion (Ar- 
yasetiawan and Gunnarsson, 1998). The spectral function in the cumulant expansion for a state below the Fermi level is expressed as

$$
A(k, \omega)=\frac{n_{k}}{2 \pi} \int_{-\infty}^{\infty} d t e^{i \omega t} e^{-i \varepsilon_{k} t+C(k, t)},
$$

where $n_{k}$ is the occupation number of state $k, \epsilon_{k}$ is the single-particle eigenvalue, and $C(k, t)$ is the cumulant operator, which can be derived in different ways (Hedin, 1980). ${ }^{21}$ The cumulant expansion contains only bosontype diagrams describing emission and reabsorption of plasmons; it does not contain diagrams corresponding to the interaction between a hole and particle-hole pairs. For this reason, the cumulant expansion primarily corrects the satellite description, whereas the quasiparticle energies are to a large extent determined by $G W$. This approximation has been applied successfully to describing the multiple plasmon satellite structure in the spectra of alkali metals (Aryasetiawan et al., 1996) as well as to substantially improving the calculated electron momentum spectroscopy of graphite in regions of energy where no intensity is predicted from the LDA band structure (Vos et al., 2001).

\section{GREEN'S FUNCTIONS IN PRACTICE}

From the previous subsection it emerges that the way quasiparticle energies and electron-hole excitations should be calculated is not unique. In practice schemes have been designed which allow one to obtain satisfactory results in many applications. An important variable in this strategy is the starting point of the calculations, i.e., one has to find the charge density (and often the lattice parameters and other ground-state properties) and some suitable one-electron eigenvalues and eigenfunctions which allow construction of a starting oneparticle Green's function. This task can be successfully carried out using the Hartree-Fock approximation in cases like atoms and molecules where screening is weak, or, most often, starting from static density-functional theory. In the following we shall summarize how the calculations are done in practice.

\section{A. Calculations of one-particle excited states}

First-principles $G W$ calculations are today standard practice in many solid-state theory groups. Several reviews on quasiparticle calculations and the derivation and analysis of Hedin's equations have been published in recent years (e.g., Aryasetiawan and Gunnarsson, 1998; Aulbur et al. 1999; Hedin, 1999) and we refer the

\footnotetext{
${ }^{21}$ The simplest way is to identify the cumulant expansion $\left\{G(k, t)=i e^{-i \varepsilon k t+C(k, t)}=G_{0}(k, t)\left[1+C(k, t)+\frac{1}{2} C^{2}(k, t)\right.\right.$ $+\cdots]\}$ with the self-energy expansion of the Green's function $\left(G=G_{0}+G_{0} \Sigma G_{0}+G_{0} \Sigma G_{0} \Sigma G_{0}+\cdots\right)$. In practice, using the $G W$ approximation for the self-energy and a $G_{0}$ determined from a DFT calculation, the cumulant is obtained equating $G_{0} C=G_{0} \Sigma G_{0}$ with $\Sigma=\Sigma_{G W}-V_{x c}$.
}

reader to those for further details. The fundamental review by Hedin and Lundqvist (1969) focuses on the electron gas and contains a comprehensive introduction to all concepts and physical quantities and the connection to measurable spectra. In the recent reviews by Aryasetiawan and Gunnarsson (1998) and Aulbur et al. (1999) the successes and shortcomings of the $G W$ approximation and numerical implementations (plane waves, local orbital basis, real-space/imaginary-time) and tricks to improve the computational efficiency are described, together with a detailed compilation of results obtained for semiconductors, transition-metal oxides, fullerenes, superlattices, interfaces, surfaces, defects, Schottky barriers, and atoms and molecules. Apart from their importance on their own, quasiparticle energies also serve as an input to further spectroscopy calculations, in particular for the Bethe-Salpeter equation approach. Therefore in the following we outline the way $G W$ calculations are usually performed and give some illustrative examples.

\section{GW calculations}

A $G W$ calculation requires in principle solution of Eq. (3.16) for the quasiparticle energies and amplitudes, taking for $\Sigma$ the product of the Green's function $G$ and the screened Coulomb interaction $W$. The complexity of $G W$ leads, in practical applications, to approximations in the construction of $G$ and $W$, which we shall examine in the following.

\section{a. Evaluation of the Green's function $G$}

In practice $G$ is constructed using single-particle orbitals from a Kohn-Sham (or, less often, from a HartreeFock) calculation. One considers the single-particle orbitals and eigenvalues as a zeroth-order approximation to the quasiparticle amplitudes and energies (see Hedin, 1995, for a discussion of this issue. This suggests the possibility of looking for a first-order, perturbative solution of Eq. (3.16) with respect to $\left(\Sigma-V_{x c}\right)$ or to $\left(\Sigma-\Sigma_{x}\right)$, where $V_{x c}$ is the exchange-correlation potential of Kohn-Sham (or $\Sigma_{x}$ is the exchange potential of HartreeFock). This is the approach followed in many practical $G W$ calculations for real systems. ${ }^{22}$ The diagonal matrix elements of $\left(\Sigma-V_{x c}\right)$ give the quasiparticle energies as

$$
\epsilon_{i}^{Q P}=\epsilon_{i}^{K S}+Z_{i}\left\langle\phi_{i}^{K S}\left|\Sigma\left(\epsilon_{i}^{K S}\right)-V_{x c}^{K S}\right| \phi_{i}^{K S}\right\rangle,
$$

with $Z_{i}^{-1}=1-\left\langle\phi_{i}^{K S}|d \Sigma / d \epsilon| \epsilon_{i}^{K S} \mid \phi_{i}^{K S}\right\rangle$. In the case of simple bulk semiconductors, this approximation turns out to be very close to the exact result obtained by diagonalizing $H_{H}+\Sigma_{G W}$ (but without any self-consistent update of $G$ and $W$; Hybertsen and Louie, 1986). In other cases, such as transition metals and finite and lowdimensional systems, the validity of the perturbative approach in $\Sigma-V_{x c}$ should be checked explicitly. For in-

\footnotetext{
${ }^{22}$ The solution of the exact local Kohn-Sham exchange has also been used as input for the $G W$ quasiparticle calculation (Aulbur et al., 2000). The quasiparticle corrections seems to be smaller in this case.
} 
stance, Pulci et al. (1999) have shown that for the (110) surface of GaAs, even if quasiparticle energies are not altered when doing the full diagonalization of the quasiparticle Hamiltonian, the quasiparticle wave functions corresponding to nearly degenerate states, with different degrees of surface/bulk localization, strongly hybridize, making the quasiparticle wave functions significantly different from the LDA ones. Differences between LDA and $G W$ wave functions have also been found in the case of $\mathrm{SiH}_{4}$ for states crossing the level of zero potential (Rohlfing and Louie, 2000). In general, differences between LDA and quasiparticle wave functions can at least be expected in any nonbulk system, i.e., when there are regions of space where the density goes to zero. In these regions, LDA produces an exchange-correlation potential with an incorrect asymptotic behavior (e.g., missing the $-1 / r$ tail in the case of atoms and clusters); hence, LDA wave functions may have a wrong spatial localization. The $G W$ potential, on the other hand, does have the correct asymptotic behavior (Charlesworth, et al., 1993; Garcia-González and Godby, 2002). ${ }^{23}$

\section{b. Evaluation of the screened Coulomb interaction $W=\varepsilon^{-1} v$}

One of the main difficulties is the evaluation of the full dielectric response of the system. In fact, even within the chosen zeroth-order scheme, i.e., within RPA-LDA, the calculation of $\varepsilon^{-1}\left(\mathbf{r}, \mathbf{r}^{\prime}, \omega\right)$ remains a difficult task from a numerical point of view (due to the spatial nonlocality and frequency dependence of $\varepsilon$ ). For this reason, approximated schemes have been developed:

- The model dielectric function (Levine and Louie, 1982; Hybertsen and Louie, 1988; Bechstedt, Del Sole, et al., 1992). These approaches allow one to considerably reduce the computational effort or even to avoid completely the $a b$ initio calculation of $\varepsilon$.

- The plasmon-pole approximation (Hybertsen and Louie, 1986; von der Linden and Horsch, 1988; Hamada et al., 1990; Engel and Farid, 1993). Most models are based on the observation that $\varepsilon\left(\mathbf{G}, \mathbf{G}^{\prime}, \omega\right)^{-1}$ is generally a peaked function in $\omega$ that can be approximated by a single-pole function in $\omega$. The pole position and strength are determined by imposing sum rules (Hybertsen and Louie, 1986), or by fitting each element $\varepsilon^{-1}\left(\mathbf{G}, \mathbf{G}^{\prime}\right)$ at two points $\omega$ along the imaginary energy axis (Godby and Needs, 1989). Since the evaluation of $\Sigma$ involves an integration over the energy, the fine details of the energy dependence are not critical, and the plasmon-pole approximation turns out to work reasonably well. One important drawback is that quasiparticle lifetimes cannot be computed within a plasmon-pole approximation, as $\operatorname{Im}(\Sigma)$ is zero everywhere except at the pole. The validity of the plasmon-pole approximation has not been system-

\footnotetext{
${ }^{23}$ One recent $G W$ application touching this point is the work of White et al. (1998) on the image potential at the $\mathrm{Al}(111)$ surface.
}

atically tested, except in a very few cases (see, for example, Aulbur et al., 1999; Arnaud and Alouani, 2000).

When the full energy dependence of the dielectric matrix is retained, the integration in $\omega$ may be performed along the imaginary axis, where $\varepsilon^{-1}$ is well behaved (Godby et al. 1988; Schöne and Eguiluz, 1998), by picking up all the poles along the real axis (Aryasetiawan, 1992; Fleszar and Hanke, 2000), or by using the transition-space spectral representation of $\varepsilon^{-1}$, which allows one to perform the frequency integration analytically (Shirley and Martin, 1993). ${ }^{24}$

\section{c. Application of the self-energy $\Sigma$}

For many applications, the simple prescriptions described above have yielded results within $10-15 \%$ of the experimental ones, the typical example being the direct quasiparticle gap of diamond, as discussed in the introduction. An agreement of the same quality between quasiparticle energies and photoemission or inverse photoemission data has also been obtained for more complex systems like surfaces, atoms, and nanostructures (Aulbur et al., 1999), and the $G W$ calculations are systematically used to study quasiparticle excitations in realistic systems of practical interest. For example, in the case of an ethylene molecule $\left(\mathrm{C}_{2} \mathrm{H}_{4}\right)$ adsorbed on the $\mathrm{Si}(001)-(2 \times 1)$ surface $G W$ seems to improve the calculated tunneling currents measured in scanning tunneling microscopy (Rignanese et al., 2001). ${ }^{25}$ A recent $G W$ study on $\mathrm{YH}_{3}$ illustrates the important consequences of self-energy corrections (Miyake et al., 2000; van Gelderen et al., 2000). It turns out that $G W$ corrections remove the band overlap responsible for erroneous metallic LDA behavior in $\mathrm{YH}_{3}$ that has a measured optical gap of $2.8 \mathrm{eV}$. Hence the gap is of electronic origin rather than structural, and not due to strong correlation (Eder et al., 1997; $\mathrm{Ng}$ et al., 1997).

Another physical quantity that can be obtained from a knowledge of the full, complex self-energy $\Sigma$ is the quasiparticle lifetime, i.e., the electron-electron scattering contribution to the linewidth. The damping rate of an excited electron in the state $\psi_{0}(\mathbf{r})$ with energy $E$ is in fact obtained as (Echenique et al., 2000)

\footnotetext{
${ }^{24}$ Mixed-space (Blase et al., 1995) and real-space/imaginarytime techniques (Rojas et al., 1995), as well as a technique to eliminate the unoccupied state summations (Reining et al., 1997), can also be used to reduce the computational effort (see the reviews by Aulbur et al., 1999; and Aryasetiawan and Gunnarsson, 1998, for a detailed comparison of different numerical implementations).

${ }^{25}$ Similar studies concerning the interpretation of scanning tunneling microscopy images have also been performed in the framework of the LDA+ U approach [see, for example, the work by Dudarev et al. (1997) on the $\mathrm{NiO}(100)$ surface]. We refer the reader to Anisimov et al. (1997) for a description of the relation between $G W$ and $\mathrm{LDA}+\mathrm{U}$.
} 


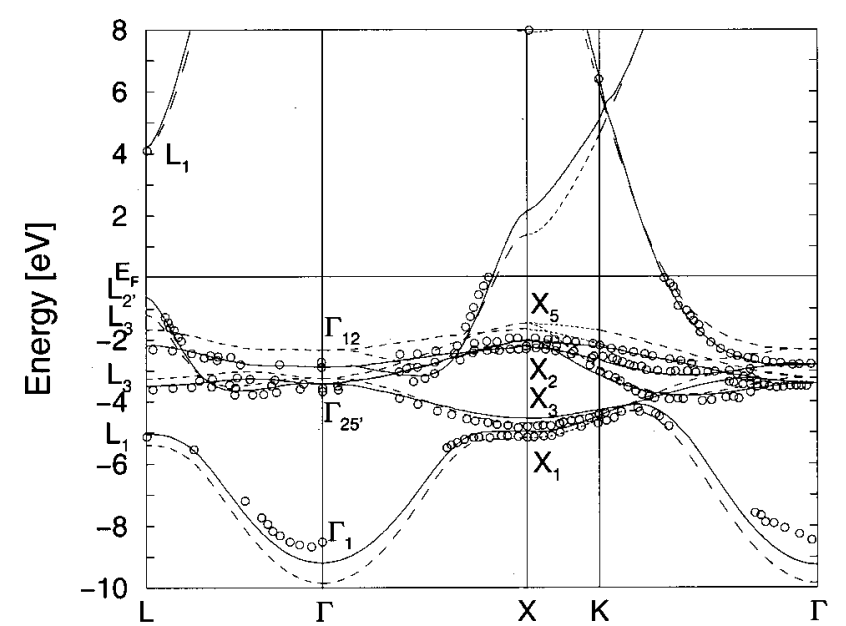

FIG. 2. Comparison of the calculated and experimental band structures for copper: solid line, $G W$ quasiparticle excitation energies (Marini et al., 2002); dashed line, DFT-LDA eigenvalues; $\bigcirc$, experimental data compiled by Courths and Hüfner (1984). A comparison to more recent experimental data (Strocov et al., 1998, 2001) yields the same agreement.

$$
\tau^{-1}=-2 \int d \mathbf{r} \int d \mathbf{r}^{\prime} \psi_{0}^{*}(\mathbf{r}) \operatorname{Im} \Sigma\left(\mathbf{r}, \mathbf{r}^{\prime}, E\right) \psi_{0}\left(\mathbf{r}^{\prime}\right) .
$$

In the $G W$ approximation, the imaginary part of $\Sigma$ is determined by the imaginary part of $\varepsilon^{-1}$ that contains all decay processes of the initial state. Quasiparticle damping times were calculated for diamond by Strinati et al. (1980, 1982), who found results consistent with photoemission data. A treatment of the electron dynamics, including band structure and dynamical screening effects, is necessary for quantitative comparisons with experiment (see, for instance, Bürgi et al., 1999; Valla et al., 1999b). An illustrative example is given by Campillo et al. (1999; Campillo, Pitarke, et al., 2000; Campillo, Rubio, et al., 2000) and Gerlach et al. (2001), who evaluated the quasiparticle lifetimes of electron and holes in bulk $\mathrm{Cu}$ within the $G W$ scheme, showing an increase in the lifetime close to the Fermi level as compared to the predictions of a free-electron-gas model of the solid. However, the lifetimes get closer to those of a free-electron gas for hole energies below $\sim 3 \mathrm{eV}$, whereas the experimental data show a distinct asymptotic behavior. This discrepancy between theory and experiment may be a signature of important departures of the DFT-LDA band structure of $\mathrm{Cu}$, which is used in the calculation of $W$, from the actual quasiparticle band structure (see Fig. 2; Marini et al., 2002).

$G W$ results for $\mathrm{Cu}$ agree with photoemission data within $30 \mathrm{meV}$ for the highest $d$ band, correcting $90 \%$ of the LDA error. The energies of the other $d$ bands throughout the Brillouin zone are reproduced within 300 $\mathrm{meV}$, and the maximum error $(\simeq 600 \mathrm{meV})$ is found for the bottom valence band at the $\Gamma$ point, where only $50 \%$ of the LDA error is corrected. This level of agreement for the $d$ bands cannot be obtained without including self-energy exchange contributions coming from $3 s$ and
$3 p$ atomic levels, ${ }^{26}$ demonstrating the importance of those contributions. On the other hand, the total bandwidth is still larger than the measured one. This overestimate of the $G W$ bandwidth with respect to the experimental one seems to be a quite general feature, which is not yet properly understood. (See Yasuhara et al., 1999, for a discussion about $\mathrm{Na}, \mathrm{Ku}$ et al., 2000 for a comment, and Yasuhara et al., 2000 for reply.)

Fully self-consistent $G W$ calculations have been performed by Schöne and Eguiluz (1998) for crystalline silicon and potassium, with the conclusion that for real materials, self-consistent $G W$ without vertex corrections in $\Sigma$ yields quasiparticle energies and bandwidths that disagree with experiment; for example, the absolute gap of Si turns out to be $1.91 \mathrm{eV}$, compared with the experimental $1.17 \mathrm{eV}$. On the other hand, Massidda et al. (1995) have calculated self-consistently the electronic structure of $\mathrm{MnO}$, using a frequency-independent model $\Sigma$ derived by Gygi and Baldereschi (1989). An encouraging agreement was found with experiment concerning the energy gap, magnetic moment, bandwidth, and spectral distribution of $\mathrm{Mn} 3 d$ states. The fact that selfconsistency leads to poorer results in the calculations of Schöne and Eguiluz (1998), but leads to an improvement in the calculations of Massidda et al. (1995) shows that the dynamical aspect of the self-energy is very problematic in this context.

Few works including vertex corrections exist to date on real materials. One example is given by the $G W \Gamma$ calculation on silicon, described in Sec. III.C.2, where $\Gamma$ is taken from a DFT-LDA approach. However, the effects of this approximate kernel are rather small. It is difficult to guess what the effect would be if a better (in particular more long-range) approximation of the kernel were used. The work of Ummels et al. (1998), discussed in the same section, suggests that some changes might be found.

The effects of vertex corrections can also explain why $G W$ calculations have not always been successful in describing self-energy effects, for example for $d$-electron bands (where the problem might come from strong correlation or simply from self-interaction effects, i.e., from the strong localization). A critical discussion about the extent to which the $G W$ approximation is capable of describing highly correlated systems such as $\mathrm{NiO}$ is given by Aryasetiawan and Gunnarsson (1995).

Computationally, the evaluation of $\Sigma$ as $G W$ is a hard task. ${ }^{27}$ Calculations can scale as badly as $N^{4}$, where $N$ is the number of atoms, hence reaching the limit of computer power well before DFT-LDA calculations, which usually scale as $N^{3}$.

\footnotetext{
${ }^{26}$ The relevance of semicore states in the self-energy was also pointed out by Rohlfing et al. (1995, 1998).

${ }^{27} \mathrm{~A}$ typical bottleneck is given by the summations over the empty states (Reining et al., 1997), both for the determination of the screening and for $G$. Another difficulty comes from the convergence of Brillouin zone integrals (i.e., $k$-point sampling) of functions that have a factor $|\mathbf{k}+\mathbf{G}|^{2}$ in the denominator as the exchange term (Pulci, 1998).
} 
It turns out that, whereas the "standard" prescription for $G W$ calculations yields good results for many applications, one has to be careful when effects beyond this perturbative-RPA scheme have to be included. In order to gain some insight concerning the choices to be made, it is useful to resort to the outcome of model calculations.

\section{Comparison of $G W$ and DFT results}

As mentioned in Sec. II.A, the Kohn-Sham eigenvalues, appearing as Lagrange multipliers in the minimization of the density functional, do not carry a direct physical meaning, even though they can be considered as well-defined approximations to the excitation energies (Görling, 1996; Filippi, 1997). In particular, they can be viewed as a good zero-order starting point for perturbative self-energy calculations (see Sec. IV.A.1). In fact, there is no equivalent of Koopman's theorem for the Kohn-Sham eigenvalues, and a direct comparison of the LDA energy gap of a semiconductor or insulator with the experimental (photoemission and inverse photoemission) band gap normally yields a severe underestimate, of the order of $30-50 \%$ or more (see, for example, Bechstedt, 1992). This error is corrected in a $G W$ calculation of quasiparticle energies.

For a more precise and general comparison between DFT and $G W$, one has to distinguish between the effects of the approximated exchange-correlation functional, for example LDA (which affects the total energy differences and the effective potential, hence the eigenvalues) and the effect of interpreting Kohn-Sham eigenvalues as electron addition and removal energies (see, for example, Jones and Gunnarsson, 1989). Almbladh and von Barth (1985) showed that in exact DFT the highest occupied eigenvalue (highest occupied molecular orbital level, or HOMO) does have a physical interpretation: it corresponds to the ionization potential. Hence, in exact DFT, the ionization potential could be derived directly from a single calculation for the ground state of an $N$-electron system. Unfortunately, the use of approximated exchange-correlation functionals (like LDA) quite severely affects the Kohn-Sham highest occupied level. Hence, when comparing excitation energies directly with Kohn-Sham eigenvalues, one must distinguish two cases: the highest occupied level, for which the error is due only to the LDA, and the other levels [e.g., the lowest unoccupied molecular orbital (LUMO) eigenvalue], for which, even in exact DFT, a discrepancy could be found. For example, in the Be atom (Jones and Gunnarsson, 1989), both errors are large: the LDA HOMO $(-5.6 \mathrm{eV})$ misses the ionization potential $(-9.32 \mathrm{eV})$ by almost $4 \mathrm{eV}$, and the HOMO-LUMO gap is $3.5 \mathrm{eV}$ in LDA, $3.62 \mathrm{eV}$ in exact DFT, and larger than $9 \mathrm{eV}$ experimentally. Part of the error comes from the spurious self-interaction which is contained in the LDA. Perdew and Zunger (1981) have proposed a selfinteraction-corrected approach in order to eliminate this contribution. This is also the case in realizations of DFT which allow the inclusion of the exact local exchange potential as an optimized effective potential (OEP) (defined as the functional derivative of the exact exchange energy $E_{x}$ with respect to the electron density) and which have been introduced with great success (Gross et al., 1996; Görling et al., 1999).

On the other hand, in a finite system it is possible to compute quasiparticle energies directly as energy differences, provided one is able to compute the total energy of the system with $N$ electrons in the ground state, and that of the system with $N \pm 1$ electrons in the ground state or some excited state. This scheme, known as the delta-self-consistent-field method, has been successfully used within DFT to describe bound one-electron excitations and the ionization potential of atoms and molecules (Jones and Gunnarsson, 1989).

Hence, DFT- or Hartree-Fock-based delta-selfconsistent-field calculations can be directly compared with $G W$ results. For atoms in the iron series, Shirley and Martin (1993) have shown that $G W$ yields results of a quality similar to, or slightly worse than, those computed from Hartree-Fock or local-spin-density total energy differences (Baroni, 1984; Vukajlovic et al., 1991). Small sodium clusters can be taken as another example: $\mathrm{Na}_{4}$ and $\mathrm{Na}_{6}$ vertical and adiabatic ionization potentials obtained with the delta-self-consistent-field approach (Martins et al., 1985) compare well with DFT $+G W$ results for jellium spheres (Saito et al., 1989) and for the real clusters (Onida et al., 1995; Reining et al., 2000). Similarly, Ögüt et al. (1997) studied the evolution of the quasiparticle and optical gaps of hydrogenated silicon clusters as a function of cluster size. Delta-selfconsistent-field LDA calculations were used to estimate the quasiparticle correction to the HOMO-LUMO gap. Ögüt et al. concluded that quantum confinement, as well as reduction of screening due to finite size, leads to appreciable excitonic corrections. This correction turns out to be large and size dependent. The delta-self-consistent field results point out that for finite systems the Hartree relaxation of the wave functions when the electron is added or removed is essential, while it is of no relevance for delocalized states in bulk solids, as pointed out by Godby and White (1998; see also the reply of Ögüt et al., 1998). In infinite systems, the correlation part takes over in relevance (see the discussion in Sec. VI.C).

Finally, another way to overcome the "gap problem" without performing a self-energy calculation has been proposed by Mackrodt et al. (1996): Hartree-Fock calculations on nickel oxide $(\mathrm{NiO})$ and on the lithium-doped material have allowed the determination of the band gap of the former, by taking eigenvalue differences between two empty states of the latter. Simply taking the difference between Hartree-Fock eigenvalues of an occupied and an empty state of $\mathrm{NiO}$ would lead to far too large a gap.

In conclusion, Kohn-Sham eigenvalues are generally in disagreement with photoemission and inverse photoemission energies, both for finite and for extended systems, with errors of $30-50 \%$ or more. On the other hand, self-energy-corrected quasiparticle energies (obtained via the $G \mathrm{~W}$ method) usually agree well with photoemission and inverse photoemission data: the reported 
errors are typically in the range of $10-15 \%$ of the band energies measured with respect to the Fermi level, hence being in absolute value larger for systems with a larger gap. In the case of finite systems, the delta-selfconsistent-field method also yields accurate excitation energies, since wave-function localization allows for finite relaxation effects. In infinite systems with delocalized wave functions the delta-self-consistent-field method is inadequate, since Hartree relaxation effects vanish and correlation must be taken into account. $G W$ contains both relaxation and correlation and is therefore suitable to describe electron addition and removal energies.

In the next section we shall discuss the use of Green'sfunction theory for the study of two-particle excited states, such as the electron-hole pairs that are created in absorption measurements. For an overview of earlier and some more recent work, see Hanke et al. (1983) and Rohlfing and Louie (2000), respectively.

\section{B. Calculations of two-particle excited states}

The key quantity in the calculation of two-particle excited states is the polarizability $P$, as introduced in Eq. (1.3).

\section{Independent-quasiparticle approximation}

As discussed in Sec. III.B, $P$ is given by Eq. (3.24). In Sec. III.C we defined the RPA form $P=-i G G$ [Eq. (3.26)], from which the screened interaction in $G W$ calculations is usually obtained. In principle, Eq. (3.26) could also be used for the calculation of absorption spectra and other spectra that involve the creation of electron-hole pairs. $P$ is then just the product of two one-particle Green's functions. Using Eq. (3.4) and after a Fourier transformation from time to frequency space, one obtains for the independent-(quasi)particle $P$ the result (1.4) in its time-ordered form, from which the retarded version can be deduced. As in the case of the one-particle Green's function, the irreducible polarizability $P$ (which is derived from a two-particle Green's function) can be understood in its Lehmann representation or in the complex plane. In the second case, which is the representation used in practical applications, the electron and hole energies entering the denominator can be complex, leading to a finite lifetime of the twoparticle excitation (because of the finite lifetime of the electron and/or the hole). Additional deexcitation channels are included when the electron-hole interaction is taken into account; this will be discussed below.

Formula (1.4) is at present still the standard expression used for many $a b$ initio calculation of optical spectra of real materials. Note that, whereas the form of $P$ is prescribed by Eq. (3.26), the iteration approach is again not uniquely defined concerning the ingredients. Hence the Green's functions entering Eq. (3.26) may be considered to stem, for example, from a Kohn-Sham or from a $G W$ calculation. In practice, most often DFT Green's functions are used when the approximation $P=P_{I Q P}$ [see Eq. (1.4)] is made. This can be understood as an approximation to the Green's-function iteration scheme, but also as an approximation to TDDFT, as will be explained in Sec. V. Sometimes a mixed DFT- $G W$ Green's function is used; the eigenvalues entering $G$ are taken to be $G W$ ones, whereas the wave functions are KohnSham orbitals. This approach (called $G W$-RPA in the introduction) was first tried in order to overcome the redshift of DFT-based absorption spectra with respect to experiment, but in general it overcorrects, and, moreover, does not improve line shapes. ${ }^{28}$ Today, $G W$-RPA is mostly seen as a good approximation to the firstiteration result for $P_{I Q P}$, which is then used to include $\Gamma$ in the second iteration, i.e., it is the starting point of the Bethe-Salpeter approach, which will be outlined in the following. We shall concentrate on absorption spectra, i.e., on the calculation of $\bar{P}$ [Eq. (2.9)], where going beyond the RPA gives the most visible effects. All results can be easily generalized to the case in which one is instead interested in $\chi$, replacing the local field effects contribution $\bar{v}$ by $v$ (see Sec. II.B).

\section{Electron-hole attraction}

The inclusion of vertex corrections [i.e., the inclusion of $\Gamma$ in Eq. (3.24) for $P$ ] can be achieved through a second iteration of Hedin's equations. This means that one has to go again from Eq. (3.22) to Eq. (3.25), now using $\Sigma=i G W$ in the latter. This yields an integral equation for $\Gamma$,

$$
\begin{aligned}
\Gamma(123)= & \delta(12) \delta(13) \\
& +i W\left(1^{+} 2\right) \int d(67) G(16) G(72) \Gamma(673) .
\end{aligned}
$$

Here, the approximation $\delta \Sigma / \delta G=i W$ is used. This means that (i) one neglects the term $i G(\delta W / \delta G)$, which contains information about the change in screening due to the excitation and is considered to be small, and that (ii) Eq. (3.32), and not Eq. (3.30), has been used. As discussed above, this latter choice in the iteration scheme can be crucial when only one or a few iterations are performed.

One can transform Eq. (4.3) to an integral equation for a generalized ${ }^{3} P$, defined as

$$
{ }^{3} P(312) \equiv-i \int d(67) G(16) G(72) \Gamma(673),
$$

by multiplying with $-i G(41) G(25)$ on the left and integrating over $d(12)$ :

$$
\begin{aligned}
{ }^{3} P(345)= & -i G(43) G(35) \\
& +i \int d(12) G(41) G(25) W\left(1^{+} 2\right){ }^{3} P(312) .
\end{aligned}
$$

\footnotetext{
${ }^{28}$ It does, however, yield rather good static dielectric constants (Levine and Allan, 1989). For silicon and germanium, the error decreases by one order of magnitude when $G W$ instead of LDA eigenvalues are used.
} 


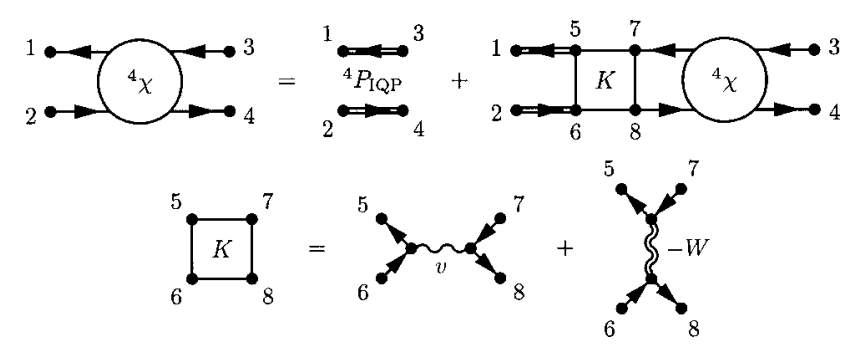

FIG. 3. Feynman diagrams representing the Bethe-Salpeter equation for $\chi$.

The polarization $P$ can hence in principle be obtained from $P(31)={ }^{3} P(311)$. However, the kernel $G G W$ of the integral equation (4.5) is a four-point function. Therefore, as outlined in Sec. II.C, it is equally convenient to introduce a four-point ${ }^{4} P$, which has the advantage that local field effects can be included on the same footing, i.e., one can directly obtain $\bar{P}$ (or $\chi$ ). In fact, closely following the prescriptions in Sec. II.C, one can introduce the four-point dynamically screened interaction

$$
{ }^{4} W(1234) \equiv W(12) \delta(13) \delta(24) .
$$

Then one gets for Eq. (4.5) the four-point integral equation

$$
{ }^{4} P={ }^{4} P_{I Q P}-{ }^{4} P^{4} W^{4} P_{I Q P},
$$

with the obvious generalization from $P_{I Q P}$ to ${ }^{4} P_{I Q P}$ [see Eqs. (1.4) and (B16)]. One can now also write Eq. (2.10) for four-point quantities, namely,

${ }^{4} \bar{P}(1234)={ }^{4} P(1234)$

$$
\begin{aligned}
& +\int d(5678){ }^{4} P(1256) \delta(56) \delta(78) \bar{v}(57) \\
& \times{ }^{4} \bar{P}(7834)
\end{aligned}
$$

Putting these equations together, one obtains the BetheSalpeter equation for ${ }^{4} \bar{P}$ :

$$
{ }^{4} \bar{P}={ }^{4} P_{I Q P}+{ }^{4} P_{I Q P} K{ }^{4} \bar{P}
$$

where the kernel $K$ contains an electron-hole exchange contribution $\bar{v}$ and electron-hole attraction $-W$ :

$$
K(1234)=\delta(12) \delta(34) \bar{v}(13)-\delta(13) \delta(24) W(12) .
$$

The equation for the corresponding ${ }^{4} \chi$ differs only by the fact that $v$ instead of $\bar{v}$ appears. The diagrams representing the Bethe-Salpeter equation for $\chi$ are shown in Fig. 3. The Green's-functions lines are dressed, i.e., they are quasiparticle lines.

One can make an immediate connection to the discussion of the four-point equations in Sec. II.C. In fact, most often $W$ is taken to be the statically screened Coulomb interaction. Equation (4.10) for $K$ corresponds then to the time-dependent screened Hartree-Fock approximation. $^{29}$ In particular, one can follow the scheme of Sec. II.C and Appendix B.2, and derive the effective two-particle Hamiltonian $H^{2 p}$ defined in Eq. (2.22), which is often used in the context of the BetheSalpeter equation approach. In fact, when static screening is used in $W$ the effective two-particle equation takes the particularly simple, energy-independent form,

$$
\begin{gathered}
\sum_{n_{3} n_{4}}\left\{\left(\epsilon_{n_{2}}-\epsilon_{n_{1}}\right) \delta_{n_{1} n_{3}} \delta_{n_{2} n_{4}}+\left(f_{n_{1}}-f_{n_{2}}\right)\left[\bar{v}_{\left(n_{1} n_{2}\right)\left(n_{3} n_{4}\right)}\right.\right. \\
\left.\left.-W_{\left(n_{1} n_{2}\right)\left(n_{3} n_{4}\right)}\right]\right\} A_{\lambda}^{\left(n_{3} n_{4}\right)}=E_{\lambda} A_{\lambda}^{\left(n_{1} n_{2}\right)}
\end{gathered}
$$

where it is understood that matrix elements of the kernel (4.10) are taken with respect to four one-particle orbitals $\quad n_{1} \cdots n_{4}: \quad \bar{v}_{\left(n_{1} n_{2}\right)\left(n_{3} n_{4}\right)}=\left\langle n_{1} n_{2}|\bar{v}| n_{3} n_{4}\right\rangle \quad$ and $W_{\left(n_{1} n_{2}\right)\left(n_{3} n_{4}\right)}=\left\langle n_{1} n_{3}|W| n_{2} n_{4}\right\rangle$. The solution of this equation allows one to construct then the absorption spectrum from Eq. (2.23). If one considers only the resonant part of $H^{2 p}$, i.e., the part mixing only transitions of positive frequency, the resulting operator is Hermitian and its eigenstates orthogonal. Therefore one obtains the simpler formula

$\varepsilon_{M}(\omega)=1-\lim _{\mathbf{q} \rightarrow 0} v_{0}(\mathbf{q}) \sum_{\lambda} \frac{\left|\Sigma_{n_{1} n_{2}}\left\langle n_{1}\left|e^{-i \mathbf{q} \cdot \mathbf{r}}\right| n_{2}\right\rangle A_{\lambda}^{n_{1} n_{2}}\right|^{2}}{\omega-E_{\lambda}+i \eta}$.

As discussed below, this turns out to be a very good approximation for the calculation of bulk absorption spectra.

Summarizing this section, we recall that (i) the BetheSalpeter approach to the calculation of two-particle excited states is a straightforward extension of the $G W$ approach for the calculation of one-particle excited states; and (ii) it leads to an effective two-particle Hamiltonian $H^{2 p}$ which is of the general form found in time-dependent problems, as explained in the introduction. The electron-hole interaction that appears in $H^{2 p}$ has two contributions: the first involves the bare Coulomb interaction $(v$ or $\bar{v}$, depending on whether $\chi$ or $\bar{P}$ are calculated), which connects the electron and hole indexes in an exchangelike manner and is therefore also called electron-hole exchange. In order to avoid confusion, however, it should not be forgotten that this contribution stems from the density variation of the Hartree term and contains just the local field effects in the case of $\bar{v}$. The second contribution involves $W$, which connects the electron and hole indexes like a direct Cou-

\footnotetext{
${ }^{29}$ It corresponds only for the kernel $K$, because the eigenvalues entering $P_{I Q P}$ are in practice calculated in the full (dynamic) $G W$ scheme, and the eigenfunctions are approximated with LDA ones, not screened Hartree-Fock ones.
} 
2 lomb interaction and is therefore called the screened electron-hole interaction. It comes from the variation of the self-energy, in other words, from an exchangelike term in the one-quasiparticle Hamiltonian. It is this last term that is responsible for the appearance of bound states, and it can even lead to hydrogenlike spectral features in insulators.

In practice, the spin $\sigma_{n}$ is most often not considered explicitly. One then makes use of the fact that only $(v, c)$ transitions with $\sigma_{v}=\sigma_{c}$ (i.e., singlet excitons) contribute to the optical spectrum. Since $W$ does not contain a spin interaction, and because of the way the $\delta$ functions connect indexes, the matrix elements $W_{(v c)\left(v^{\prime} c^{\prime}\right)}$ are diagonal in spin, i.e., $\sigma_{v}=\sigma_{v^{\prime}}$. Instead, the matrix elements $\bar{v}_{(v c)\left(v^{\prime} c^{\prime}\right)}$ are independent of $\sigma_{v}$ and $\sigma_{v^{\prime}}$. Hence spin is implicitly included via a factor of 2 for $\bar{v}$ in Eq. (4.11).

\section{The effects of the electron-hole interaction}

Exciton calculations for real systems within a fully first-principles scheme, starting from DFT, have recently been performed. However, the importance of the excitonic effects has been known for a long time, even in cases in which the features are less characteristic than, the appearance of a hydrogenic series of bound states in the band gap. Excitonic effects in the absorption line shape of semiconductors above the fundamental gap were calculated some time ago (Hanke and Sham, 1974, 1975, 1980; del Castillo and Sham, 1985). Hanke and Sham (1980) solved the Bethe-Salpeter equation [Eq. (4.9)] for the particle-hole response function of bulk silicon, using a linear combination of atomic orbitals basis, with a semiempirical band structure fitted to optical experiments. They found important corrections to the independent-particle result, in particular a strong increase in the lowest main absorption peak (E1), leading for the first time to a calculated absorption spectrum of bulk silicon in qualitative agreement with experiment.

The $a b$ initio approaches used today for solution of the Bethe-Salpeter equation (Albrecht et al., 1998a, 1998b; Benedict et al., 1998a; Rohlfing and Louie, 1998b) mostly follow the scheme introduced by Onida et al. (1995) for the spectrum of the cluster $\mathrm{Na}_{4}$ and that of Albrecht et al. (1997) for the optical gap of bulk $\mathrm{Li}_{2} \mathrm{O}$. This procedure consists of (i) a ground-state DFT calculation; (ii) a $G W$ calculation to correct the eigenvalues; and (iii) solution of the Bethe-Salpeter equation using $G W$ eigenvalues, Kohn-Sham orbitals, and static RPA screening for the electron-hole interaction. It thus includes several steps and choices, which are schematically represented by the flow diagram in Fig. 4.

This scheme, using the approach of the effective twoparticle Hamiltonian, has again been applied to the calculation of the absorption spectrum of bulk silicon (Albrecht et al., 1998a), showing that the Bethe-Salpeter method used without any adjustable parameter can yield

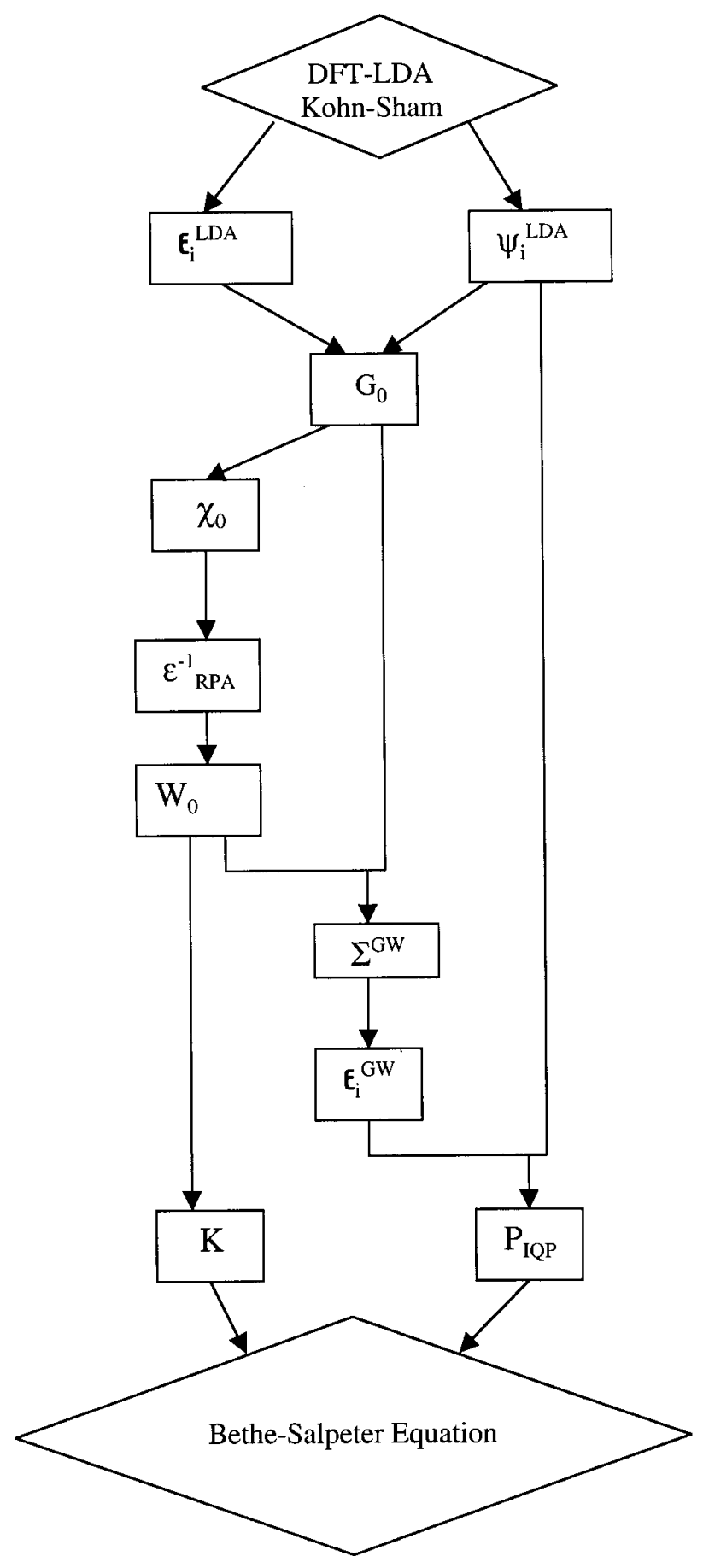

FIG. 4. Flow diagram sketching the solution of the BetheSalpeter equation in practice.

absorption spectra of continuum excitons in bulk semiconductors in quantitative agreement with experiment (see Fig. 5). ${ }^{30}$

On the other hand, as pointed out above, the electronhole attraction can also lead to bound excitons in insu-

\footnotetext{
${ }^{30}$ The present curve has been calculated by Olevano and Reining (2000) using an improved Brillouin-zone sampling with respect to the original publication of Albrecht et al. (1998a). See also the discussions by Cardona et al. (1999) and Albrecht et al. (1999).
} 


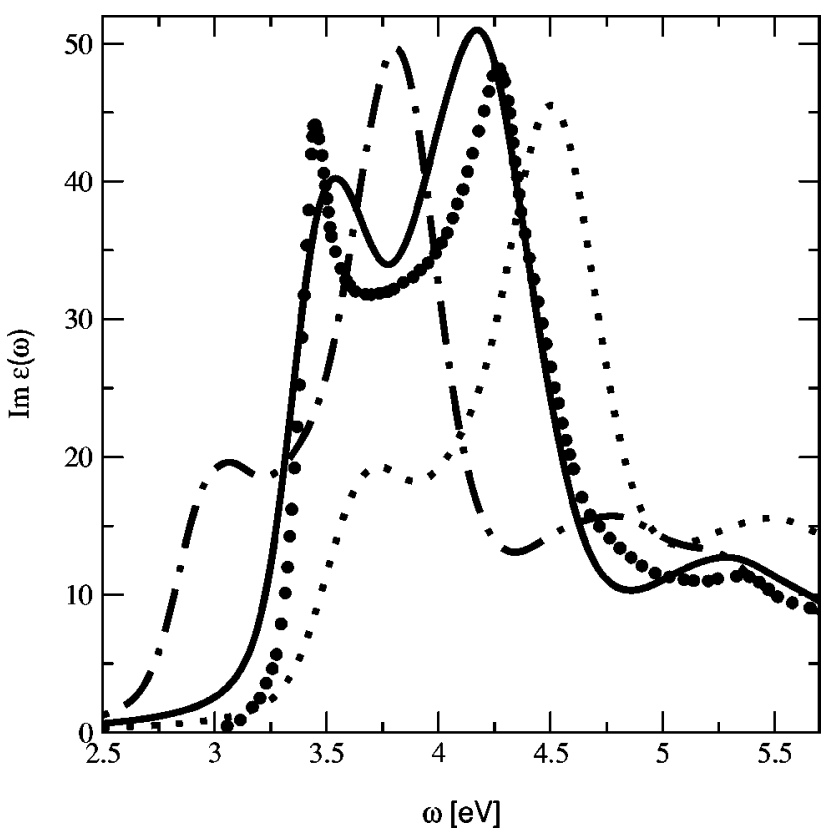

FIG. 5. Silicon absorption spectrum $\left[\operatorname{Im}\left(\varepsilon_{M}\right)\right]$ : $\bullet$, experiment (Lautenschlager et al., 1987); dash-dotted curve, RPA, including local field effects; dotted curve, $G W$-RPA; solid curve, Bethe-Salpeter equation.

lators, where the interaction is only weakly screened. The resulting spectra can also be well described by the $a b$ initio Bethe-Salpeter approach, as has been illustrated by the work of Benedict et al. (1998a). These authors use a recursive method (Haydock, 1980) to invert the Bethe-Salpeter equation (4.9), which has the advantage that one can make use of the $\delta$ functions in the $W$ contribution to the kernel in order to make calculations less cumbersome. ${ }^{31}$ Figure 6 shows the result for bulk LiF (Shirley, 2001). ${ }^{32}$ The sharp peak in the absorption spectrum at about $12 \mathrm{eV}$ is clearly due to excitonic effects-the spectrum without excitons is essentially featureless in the range of the absorption onset. The 12-eV peak defines the optical gap, which turns out to be only about $0.5 \mathrm{eV}$ lower than the experimental one, whereas the independent-quasiparticle result shows a considerable overestimate.

The same approach has also been used by Benedict et al. (1998b) to obtain the absorption spectra of bulk Si, $\mathrm{C}, \mathrm{Ge}$, and GaAs. Moreover, they have studied the wide-gap semiconductor $\mathrm{GaN}$ (in its wurtzite and zincblende structures), and the insulating $\mathrm{CaF}_{2}$ crystal (Benedict and Shirley, 1999). In all cases, the inclusion of excitonic effects turned out to be crucial for a quantitative agreement between theory and experiment. Rohlfing and Louie (1998b) have introduced a different way of extending the applicability of the Bethe-Salpeter

\footnotetext{
${ }^{31}$ The calculations are simplified further by the use of a model electron-hole screening.

${ }^{32}$ Shirley's calculation is the same as that of Benedict et al. (1998a), but with an improved Brillouin-zone sampling and including more bands.
}

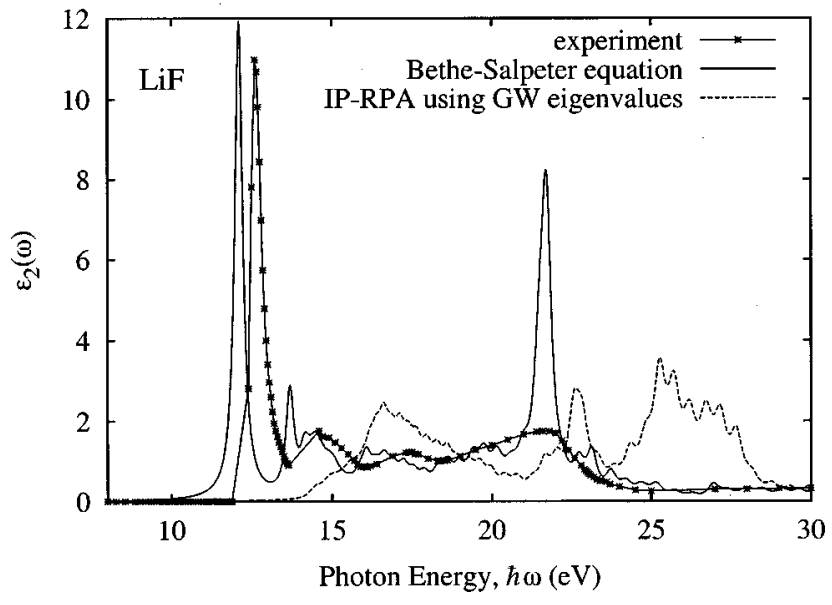

FIG. 6. LiF, absorption spectrum $\left[\varepsilon_{2}=\operatorname{Im}\left(\varepsilon_{M}\right)\right]$. Continuous curve with crosses, experimental (Roessler and Walker, 1967). Dashed curve, Eq. (2.8) using $G W$ eigenvalues. Plain continuous curve, Bethe-Salpeter equation (Shirley, 2001).

approach: they noticed that one of the bottlenecks in the calculation of bulk spectra is the determination of the $\left(N_{v} \times N_{c} \times N_{k}\right)^{2}$ matrix elements of $H_{(v k, c k)\left(v^{\prime} k^{\prime}, c^{\prime} k^{\prime}\right)}^{2 p}$, where $N_{v}$ and $N_{c}$ are the numbers of valence and conduction bands that have to be taken into account in the construction of the spectrum, and $N_{k}$ is the number of $k$ points at which the direct $v k \rightarrow c k$ transitions are considered. They hence proposed an interpolation scheme in $k$ for the $H_{(v k, c k)\left(v^{\prime} k^{\prime}, c^{\prime} k^{\prime}\right)}^{2 p}$, which allowed them to calculate the absorption spectrum of bulk GaAs including the extremely weakly bound exciton states (some meV of binding energy) in the gap. The description of the latter feature in fact required the use of about $1000 k$ points in a sphere of 0.015-a.u. radius around $k=0$.

Rohlfing and Louie (1998b) and Benedict and Shirley (1999) also noticed that the excitonic effects above the gap are due to the mixing of transitions given by the coefficients $A_{\lambda}^{\left(n_{1} n_{2}\right)}$ in Eq. (4.12), in other words, interference effects. This can be shown by looking at the density of excitation energies, which turns out to be almost unchanged by the inclusion of the electron-hole interaction (see, for example, Fig. 6 in Benedict and Shirley, 1999). This means that the apparent shift of peak positions [about $0.4 \mathrm{eV}$ for the $E 2$ peak in silicon (Albrecht et al., 1998a)] is not due to a negative shift of the transition energies.

Of course, this is not true in the case of bound excitons, which, apart from the above-mentioned insulators, includes systems having discrete energy levels, like atoms (Rohlfing and Louie, 2000), molecules and clusters (Onida et al., 1995; Rohlfing and Louie, 1998a), and other low-dimensional systems. In these cases, excitation energies are shifted; moreover, the effect can be very strong, since screening is not efficient and the electron and hole are localized.

A good illustration of excitons in low-dimensional systems is given by the case of conjugated polymers like trans-polyacetylene and poly-phenylene-vinylene (PPV). The optical absorption spectra of these mol- 

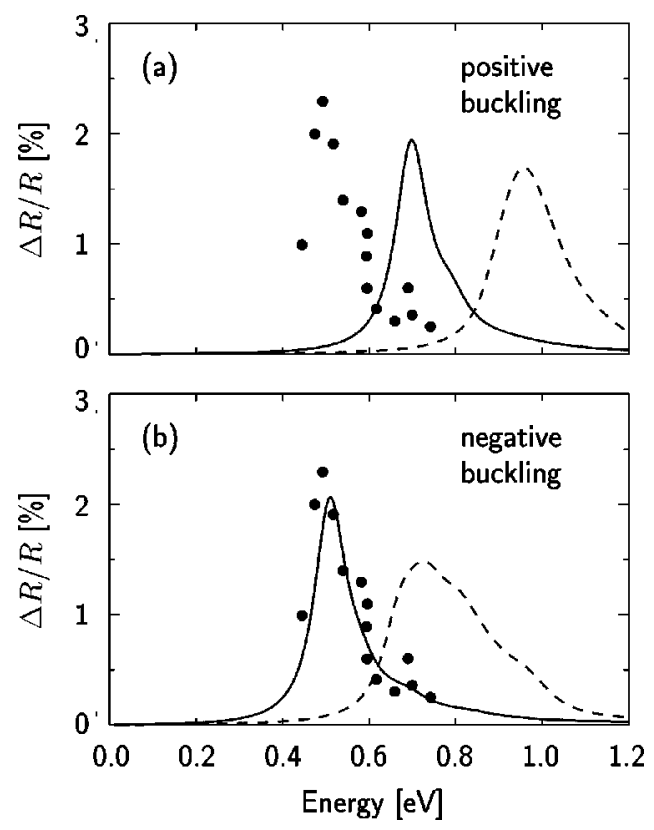

FIG. 7. Normal-incidence differential reflectivity spectrum of the $\operatorname{Ge}(111)(2 \times 1)$ surface: solid lines, the spectrum including self-energy and excitonic effects; dashed lines, spectra obtained using Eq. (2.8) and $G W$ eigenvalues. The upper panel is for the positively buckled isomer, the lower for the negatively buckled isomer. Dots are experimental data by Nannarone et al. (1980).

ecules have been studied by Rohlfing and Louie (1999a). The electron-hole interaction gives rise to exciton binding energies of the order of one $\mathrm{eV}$ and strongly modifies the spectral line shapes. Similar results for polymers have also been found by van der Horst et al. (1999) and by Ruini et al. (2002).

Surfaces can also be considered as low-dimensional systems, in particular in certain reconstructions like the quasi-one-dimensional chain structures of $\mathrm{Si}(111) 2 \times 1$ and $\mathrm{Ge}(111)(2 \times 1)$. A strong excitonic effect has been found for $\mathrm{Si}(111) 2 \times 1$ in semiempirical (Reining and Del Sole, 1991) and $a b$ initio calculations (Rohlfing and Louie, 1999b). The excellent agreement found allows one to use the theoretical results as reference spectra for the interpretation of experiments. For example, an $a b$ initio exciton calculation by Rohlfing et al. (2000) for $\mathrm{Ge}(111)(2 \times 1)$ has demonstrated how optical differential reflectivity spectra can be used to distinguish between the two possible isomers of the reconstructed surface. This distinction was made possible by the fact that a quantitative comparison between the calculated and experimental spectra is possible when electron-hole effects are treated correctly (see Fig. 7).

Rohlfing and Louie (2000) have recently published an extended paper in which their first-principles exciton absorption calculations are reviewed. They present the theoretical framework, compare different choices of basis sets, and discuss the applications to both finite systems (namely, $\mathrm{He}, \mathrm{Ne}$, and $\mathrm{Ar}$ atoms, and $\mathrm{SiH}_{4}$ and larger $\mathrm{Si}_{n} \mathrm{H}_{m}$ clusters) and infinite crystals ( $\mathrm{GaAs}, \mathrm{Si}$, $\mathrm{LiF}$, and $\mathrm{LiCl}$ ).
The importance of excitonic effects can also be seen in electron-energy-loss spectra, linked to the imaginary part of the inverse dielectric function. Recently, Soininen and Shirley (2000) applied the ab initio exciton scheme to the calculation of the dynamic structure factor $\mathrm{S}(\mathbf{q}, \omega)$ of diamond, $\mathrm{LiF}$, and wurtzite $\mathrm{GaN}$ for various momentum transfers q. As in the case of the macroscopic dielectric function, the inclusion of the electronhole interaction in $\varepsilon^{-1}$ was found to substantially redistribute the spectral weight with respect to a $G W$ (independent-quasiparticle) calculation, being crucial in interpreting the experimental dynamical structure factor over a wide range of energies. Essentially, an overall shift to lower energies and an enhancement of the intensities on the low-energy side are found. The latter effect can be traced back to the nontrivial excitonic effects in the imaginary part of the dielectric function. Concerning the overall, essentially rigid shift, one might wish to discuss in detail the extent to which the electron-hole attraction and self-energy corrections cancel in the dynamical structure factor. For the plasmon peak of silicon at vanishing momentum transfer, it has in fact been shown that strong cancellations occur (Olevano and Reining, 2001a). In other words, whereas it is clear that, at present, absorption spectra of bulk materials most often necessitate the inclusion of self-energy and electronhole attraction effects, it is less obvious how much these effects improve loss spectra in general and to what extent improvements obtained with respect to using Eq. (2.8) are essentially due to local field effects. This latter point will also be discussed in Sec. VI.D.

In conclusion, it is important to note that a large variety of problems has by now been studied via the $a b$ initio approach, ranging from absorption to energy-loss spectra; from applications to atoms, molecules, and clusters to applications to bulk materials; and showing excitonic effects as different as the continuum exciton in silicon, the very weakly bound Wannier excitons in $\mathrm{GaAs}$, or the strongly bound exciton in LiF. Models exist which allow each of these situations to be described, the two best known being the hydrogenic Wannier model for weakly bound excitons and the Frenkel model for strongly bound excitons (see Bassani and Pastori Parravicini, 1975 for an overview). In fact, certain features in the spectra can be very easily predicted by these models. In particular, the knowledge of the effective masses (band curvatures) at the direct gap, and of the dielectric constant, often yield a very good estimate of exciton binding energies and allow one to estimate strongly bound excitons like those in LiF. However, none of the simple models could ever predict an entire spectrum. Solid argon, for example, exhibits a hydrogenlike series of peaks below the continuum, the first of which is Frenkel-like, and the others of which are well described by the Wannier model. More refined model approaches manage to include both types of bound excitons (Resca et al., 1978), but are not meant to predict excitonic effects in higher parts of the spectrum, where other details of the band structure become important. Instead, the $a b$ initio approach has been shown to treat all those fea- 
tures equally well, and hence to be flexible and predictive. Of course the agreement with experiment is still somewhat limited by the various approximations that are commonly used.

In the following section, several possible choices will be discussed.

\section{Different levels of sophistication}

One of the main difficulties one encounters when discussing the quality of Bethe-Salpeter results is the fact that, as can be seen from Fig. 4, the ab initio approach is a three-step method. First, a Kohn-Sham ground-state calculation is performed. Second, $G W$ corrections are added in order to obtain correct quasiparticle energies. Third, the Kohn-Sham and $G W$ results are used in order to construct the Bethe-Salpeter equation. At each step, approximations are made and one has to be careful in order to keep the error bar in the final results small.

In the following, we shall examine a list of the main approximations that are often used.

Pseudopotentials. All above-cited ab initio calculations use pseudopotentials. The use of pseudo wave functions for the construction of transition matrix elements might, however, introduce some error in the results, even when the nonlocality of the pseudopotential is correctly taken into account. $^{33}$ The influence of strongly nonlocal pseudopotentials on the calculation of transition matrix elements has been analyzed by Read and Needs (1991), who have found differences of up to $10-15 \%$, depending on the element. See also Marini et al. (2001) for an example.

Arnaud and Alouani $(2000,2001)$ have calculated quasiparticle energies and optical spectra including self-energy and excitonic effects, using the allelectron full-potential projector-augmented wave method of Blöchl (1994). For Si, GaAs, and diamond their $G W$ band structure agrees with the available pseudopotential results to within $1 \%$ for GaAs and $\mathrm{AlAs}, 2 \%$ for $\mathrm{Si}$, and $5 \%$ for InP (Arnaud and Alouani, 2000). Optical spectra computed within the Bethe-Salpeter scheme (Arnaud and Alouani, 2001) are also in agreement to within a few percent with pseudopotential results, except for the case of diamond, where they found differences in the peak positions of the order of $0.5 \mathrm{eV}$.

(ii) Kohn-Sham-LDA wave functions. In the BetheSalpeter approach, $P_{I Q P}$ and other matrix elements are most often constructed using $G W$ eigenvalues but LDA wave functions. This has been justified by the fact that quasiparticle and KohnSham orbitals are supposed to be close to each

\footnotetext{
${ }^{33}$ Naturally, when using nonlocal pseudopotentials, the correct definition of the velocity operator must be adopted, since macroscopically wrong results can be obtained otherwise (Van Dyke, 1972; Read and Needs, 1991).
}

other (Hybertsen and Louie, 1986; Godby et al., 1986, 1988). However, this is not always the case. For example, for finite systems the missing $-1 / r$ tail of the $V_{x c}^{\mathrm{LDA}}$ potential can strongly distort the wave functions, and it may be necessary to correct their spatial behavior. This has been done by Rohlfing and Louie (2000). For the small clusters studied, the authors have shown that the wave functions of the empty states become clearly more delocalized with respect to the LDA Kohn-Sham ones. This has a sizable effect on the exciton binding energies, which are reduced by as much as $20 \%$. Note that it would be much more complicated to include exact quasiparticle wave functions. In fact, in order to obtain Eq. (4.11), the wave functions used for the construction of the matrix elements have to derive from a static operator. Otherwise, they might be nonorthogonal for different states (energies), the above basis transformation would become more complicated, and the calculation could yield different results.

(iii) Static electron-hole screening. This approximation is often justified, since the plasma frequencies of the investigated systems are much larger than the excitonic binding energies. Moreover, it has been found that dynamical effects in the electron-hole screening and in the one-particle Green's function tend to cancel each other, at least in simple semiconductors (Bechstedt et al., 1997), which suggests that both should be neglected. This might not be true in other systems where the exciton binding energy is large. Dynamical effects in the BetheSalpeter equation have been studied for core excitons by Strinati $(1982,1984)$, who derived the more general effective two-particle equation ${ }^{34}$

$$
\sum_{v^{\prime} c^{\prime}} H_{v c}^{v^{\prime} c^{\prime}}\left(E_{\lambda}\right) A_{\lambda}^{v^{\prime} c^{\prime}}\left(E_{\lambda}\right)=E_{\lambda} A_{\lambda}^{v c}\left(E_{\lambda}\right)
$$

Note that, as in the case of the one-particle excited states, it is the dielectric function that introduces the energy dependence (and hence finally the possibility of complex eigenvalues) into the equation: the two-particle excited state may now have a finite lifetime, shorter than the electron and hole lifetimes. And again, since this comes through $\varepsilon^{-1}$, energy is conserved, since one excitation is decaying by exciting others. Dynamical effects have been studied for $\mathrm{SiH}_{4}$ by Rohlfing and Louie (2000); they are found to increase the exciton binding energy by about $0.1 \mathrm{eV}$.

(iv) RPA electron-hole screening. The kernel $W$ is usually evaluated in the RPA. Since the neglect of exchange-correlation effects in the screening can lead to an underestimate of the dielectric constant, this approximation might overestimate exci-

\footnotetext{
${ }^{34}$ Strinati found that dynamical screening effects significantly narrow the core-excitation spectral width, which goes along with an increase in the exciton binding energy.
} 
ton binding energies. Chang et al. (2000) have tried to estimate the effect for $\alpha$-quartz by rescaling the electron-hole screening self-consistently, with the dielectric constant calculated including excitonic effects. In fact, they find a shift of the main absorption peak towards higher energies, closer to the experimental one. A quantitative discussion is difficult, since it should include a recalculation of the dielectric matrix for all momentum transfers and a consistent inclusion of vertex corrections (i.e., an additional vertex, because the Bethe-Salpeter equation should now be derived from $\Sigma=i G W \Gamma$ ).

(v) Neglect of resonant-antiresonant coupling. Generally, Eq. (4.12) is used for the calculation of absorption spectra. This supposes that transitions at positive (resonant part) and negative (antiresonant part) frequencies do not mix (see Appendix B.2). The effects of the resonant-antiresonant coupling on the exciton binding energy have been discussed by Zimmermann (1970) and Del Sole and Selloni (1984). Neglecting the coupling can bias the results, especially for quantities based on the real part of epsilon, like the dielectric constant or the electron-energy-loss spectra. This has been illustrated by Olevano and Reining (2001a) for the case of the plasmon resonance of silicon.

To summarize, we stress that the Bethe-Salpeter approach has up to now yielded results that agree with the measured ones within $10 \%$ for the peak positions and $20 \%$ for the peak strengths. The error bar is given by the sum of several contributions, and it has not yet been completely elucidated where the main sources of error lie. Well-generated ab initio pseudopotentials should affect the results by less than $\simeq 0.1 \mathrm{eV}^{35}$ Also, as mentioned in Sec. IV.A.2, GW transition energies may be off by $10-15 \%$, and one suspects that the quality of the $G W$ result dominates the agreement between the experimental and the theoretical peak positions. The way the screening is taken into account (e.g., with a model dielectric function, or in the static RPA) also contributes to the error bar by at least another $0.1 \mathrm{eV}$. As for present limitations of the approach, one should mention the neglect of the dynamical screening of the electron-hole interaction, which could become relevant when looking at the dielectric function at frequencies comparable with the plasma frequency, and the complete neglect of the lattice vibrations. Finally, there are the limitations due to the computational heaviness of the approach, which is in part necessarily cumbersome, since it involves four-point quantities. This point is, among others, a strong motivation to search for alternative approaches, the most prominent being the TDDFT approach, to be discussed in the next section.

\footnotetext{
${ }^{35}$ The choice of the lattice constant-experimental or theoretical—can also contribute 50-200 meV.
}

\section{TIME-DEPENDENT DENSITY-FUNCTIONAL THEORY}

Several reviews on general foundations of TDDFT and some applications have appeared recently, including those of Gross et al. (1994, 1996), Casida (1995, 1996), Dobson, Vignale, and Das (1997), Burke et al. (2002), and van Leeuwen (2001). Once more we focus the discussion below on the points that are essential for the comparisons that are within the scope of this review. In the following, we summarize the foundations of TDDFT, and its connections with excited-state properties and with many-body perturbation theory (a detailed comparison between TDDFT and the Green's-function approach will be given in Sec. VI).

\section{A. Formalism}

The Hohenberg-Kohn-Sham theory as described in Sec. II.A is a ground-state theory, and is hence not meant for the calculation of electronic excitations. However, one can extend the idea of static DFT by analogy with classical mechanics: the ground state is determined by the minimum of the total energy. When one asks for the evolution of the system under the influence of a time-dependent external potential, one should search the extrema of the quantum-mechanical action,

$$
A=\int_{t_{0}}^{t_{1}} d t\left\langle\Psi(t)\left|i \frac{\partial}{\partial t}-\hat{H}(t)\right| \Psi(t)\right\rangle,
$$

just as in classical mechanics the trajectory of a system is determined by the extrema of the action $\int_{t_{0}}^{t_{1}} d t L(t)$, where $L$ is the Lagrangian. Theorems have now been established for time-dependent DFT which are parallel to those of static DFT. Many-body effects are included in a local exchange-correlation potential, which is now time dependent and, as in the case of static DFT, is unknown (for more details see Gross et al., 1996). The first applications of TDDFT response theory were made before the formal development and relied on analogies with time-dependent Hartree-Fock theory (Stott and Zaremba, 1980; Zangwill and Soven, 1980). In the work of Runge and Gross (1984) a theory similar to that of Hohenberg, Kohn, and Sham is developed for timedependent potentials in terms of the action functional. The first theorem proves a one-to-one mapping between time-dependent potentials and time-dependent densities (that are $v$ representable); the second proves the stationary-action principle. The scheme is very similar to the ground-state Kohn-Sham formalism. The proof of the first theorem is based directly on the evolution of the time-dependent Schrödinger equation from a fixed initial many-particle state $\Psi\left(t_{0}\right)=\Psi_{0}$ under the influence of a time-dependent potential $V_{\text {ext }}(t)$ (required to be expandable in a Taylor series around $t_{0}$ ). The initial state $\Psi_{0}$ does not need to be the ground state or any other eigenstate of the initial potential. As one does not rely on the adiabatic connection in standard zerotemperature many-body perturbation theory, the formalism is able to handle external perturbations varying rapidly in time. 
By virtue of the first theorem, the time-dependent density determines the external potential uniquely up to an additive purely time-dependent function. On the other hand, the potential determines the timedependent wave function; therefore the expectation value of any quantum-mechanical operator is a unique functional of the density. This theorem has been generalized by van Leeuwen (1999) by showing that a timedependent density obtained from an initial many-body system can be, in principle, reproduced by a timedependent external potential in a many-body system with different (and possibly null) two-particle interaction. This mapping between densities and timedependent potentials in TDDFT demonstrates the noninteracting $v$ representability of a general many-body system. That is, if one takes the two-particle interaction of the second system to be zero, this theorem establishes that for a given initial state having the proper initial density and time derivative, there is a unique potential (up to a purely time-dependent function) in a noninteracting system that reproduces the given time-dependent density at all times. This result is important for the Kohn-Sham formalism of TDDFT (see below).

Moreover, in addition to their dependence on the density, the time-dependent functionals depend on the initial state $\Psi_{0} \cdot{ }^{36}$ The time-dependent particle and current density can be calculated exactly from the continuity equation and the equation of motion of the paramagnetic current-density operator $\hat{\mathbf{j}}_{p}(\mathbf{r})$ $=(1 / 2 i) \sum_{j=1}^{N}\left[\nabla_{\mathbf{r}_{j}} \delta\left(\mathbf{r}-\mathbf{r}_{j}\right)+\delta\left(\mathbf{r}-\mathbf{r}_{j}\right) \nabla_{\mathbf{r}_{j}}\right]$, that is,

$$
\begin{aligned}
& \frac{\partial \rho(\mathbf{r}, t)}{\partial t}=-\nabla \mathbf{j}(\mathbf{r}, t), \\
& \frac{\partial \mathbf{j}(\mathbf{r}, t)}{\partial t}=-i\left\langle\Psi(t)\left|\left[\hat{\mathbf{j}}_{p}, \hat{H}(t)\right]\right| \Psi(t)\right\rangle .
\end{aligned}
$$

In particular, the current density $\mathbf{j}(\mathbf{r}, t)$ following from the time-dependent Kohn-Sham orbitals and the true interacting current density may differ only by the curl of an arbitrary function (Dobson, Vignale, and Das, 1997). The second theorem deals with the variational principle of the action functional with the initial condition $\Psi\left(t_{0}\right)$ $=\Psi_{0}$. From the previous one-to-one mapping between time-dependent potentials and densities, the action [Eq. (5.1)] is a functional of the density $A[\rho]$, which must have a stationary point at the correct time-dependent density. Thus the Euler equation corresponding to the extrema of $A[\rho], \quad \delta A[\rho] / \delta \rho(\mathbf{r}, t)=0$ determines the time-dependent density, just as in the Hohenberg-Kohn formalism the static ground-state density is given by the minimum of the total energy $E(\delta E[\rho] / \delta \rho(\mathbf{r})=0)$. Similarly, one can define a time-dependent Kohn-Sham scheme by introducing a noninteracting system that re-

\footnotetext{
${ }^{36}$ In general there are many wave functions leading to the same density. Any of them will work properly, because the dependence of the effective time-dependent potential is such that the interacting density will be reproduced in each case.
}

produces the exact interacting density $\rho(\mathbf{r}, t)$. Assuming the (demonstrated) $v$ representability of the timedependent densities (van Leeuwen, 1999), one gets the following time-dependent Kohn-Sham equations:

$$
\begin{aligned}
& {\left[-\frac{1}{2} \nabla^{2}+V_{\text {eff }}(\mathbf{r}, t)\right] \psi_{i}(\mathbf{r}, t)=i \frac{\partial}{\partial t} \psi_{i}(\mathbf{r}, t)} \\
& \rho(\mathbf{r}, t)=\sum_{i=1}^{N}\left|\psi_{i}(\mathbf{r}, t)\right|^{2}
\end{aligned}
$$

where $V_{\text {eff }}(\mathbf{r}, t)=V_{H}(\mathbf{r}, t)+V_{x c}(\mathbf{r}, t)+V_{\text {ext }}(\mathbf{r}, t)$ is the effective time-dependent potential felt by the electrons. It consists of the sum of the external time-dependent applied field, the time-dependent Hartree term, plus the exchange-correlation potential (defined via the equivalence between the interacting and fictitious noninteracting systems). The variational principle yields

$$
V_{x c}(\mathbf{r}, t)=\frac{\delta A_{x c}[\rho]}{\delta \rho(\mathbf{r}, t)},
$$

where $A_{x c}[\rho]$ is the exchange-correlation part of the action functional. ${ }^{37}$

The advantage of the time-dependent Kohn-Shamscheme lies in its computational simplicity compared to other quantum-chemical methods such as timedependent Hartree-Fock or configuration interaction (Langhoff et al., 1972; Jensen, 1999). Up to here, the formalism presented deals with the quantum nature of the electrons only. The equations can be generalized to treat the quantum-mechanical coupling of the nuclear and electronic motions (Kreibich, 2000; Kreibich and Gross, 2001), the quantum nature of the electromagnetic field, and even superconductors (Gross et al., 1996; Kurth et al., 1999).

\section{B. Excitation energies in TDDFT}

Formally, TDDFT allows the calculation of the (bound and unbound) excited-state energies and transition probabilities of a many-body system, based on information gleaned from an ordinary DFT self-consistent calculation. In the time-dependent approach, one studies how the system behaves when subject to a timedependent external perturbation. In this case, the system's response is directly related to the $N$-particle excited states of an $N$-particle system, in a manner similar to the way the one-particle Green's function is re-

\footnotetext{
${ }^{37}$ As with the total energy in the static case, here the action functional has been decomposed as

$$
\begin{aligned}
A[\rho]= & \sum_{i=1}^{N} \int_{t_{0}}^{t_{1}} d t\left\langle\psi_{i}(t)\left|i(\partial / \partial t)+\frac{1}{2} \nabla^{2}-V_{e x t}(t)\right| \psi_{i}(t)\right\rangle \\
& -A_{H}[\rho]-A_{x c}[\rho],
\end{aligned}
$$

where $\psi_{i}(t)$ are the wave functions of the fictitious noninteracting Kohn-Sham system, $A_{H}$ is the time-dependent Hartree contribution $\frac{1}{2} \iint d \mathbf{r} d t \rho(\mathbf{r}, t) V_{H}(\mathbf{r}, t)$, and $A_{x c}$ includes all exchange and dynamical correlation effects due to the manybody interacting system. $A_{x c}$ is not known and has to be approximated.
} 
lated to the $(N+1)$ - and $(N-1)$-particle excited states of the same system (see Sec. III.A).

Petersilka et al. (1996) have developed a formalism for calculating the neutral excitations in finite systems. The key formula is a Dyson-like representation of the exact linear density response $\chi$ of an interacting manyelectron system in terms of the noninteracting KohnSham response $\chi_{0}$. To prove this relation, one can start from the physical definition of the retarded linearresponse function,

$$
\chi\left(\mathbf{r}, t, \mathbf{r}^{\prime}, t^{\prime}\right)=\left.\frac{\delta \rho(\mathbf{r}, t)}{\delta V_{e x t}\left(\mathbf{r}^{\prime}, t^{\prime}\right)}\right|_{V_{e x t}=0},
$$

which measures the degree to which the density responds to first order in the external potential. Equivalently, the linear response $\chi_{0}$ of the fictitious Kohn-Sham system that can be described in terms of the Kohn-Sham orbitals is given by

$$
\chi_{0}\left(\mathbf{r}, t, \mathbf{r}^{\prime}, t^{\prime}\right)=\left.\frac{\delta \rho(\mathbf{r}, t)}{\delta V_{\mathrm{eff}}\left(\mathbf{r}^{\prime}, t^{\prime}\right)}\right|_{V_{\mathrm{eff}}=0},
$$

where $V_{\text {eff }}=V_{e x t}+V_{H}+V_{x c}$. Using $\delta \rho / \delta V_{e x t}=\left(\delta \rho / \delta V_{\text {eff }}\right)$ $\times\left(\delta V_{\text {eff }} / \delta V_{\text {ext }}\right) \equiv \chi_{0} \delta V_{\text {eff }} / \delta V_{\text {ext }}$ one obtains

$$
\begin{aligned}
\frac{\delta V_{\text {eff }}(\mathbf{r}, t)}{\delta V_{\text {ext }}\left(\mathbf{r}^{\prime}, t^{\prime}\right)}= & \delta\left(\mathbf{r}-\mathbf{r}^{\prime}\right) \delta\left(t-t^{\prime}\right) \\
& +\int\left[\frac{\delta\left(t-t^{\prime \prime}\right)}{\left|\mathbf{r}-\mathbf{r}^{\prime \prime}\right|}+f_{x c}\left(\mathbf{r}, t, \mathbf{r}^{\prime \prime}, t^{\prime \prime}\right)\right] \\
& \times \chi\left(\mathbf{r}^{\prime \prime}, t^{\prime \prime}, \mathbf{r}^{\prime}, t^{\prime}\right) d \mathbf{r}^{\prime \prime} d t^{\prime \prime},
\end{aligned}
$$

where the time-dependent exchange-correlation kernel

$$
f_{x c}\left(\mathbf{r}, t, \mathbf{r}^{\prime}, t^{\prime}\right)=\left.\frac{\delta V_{x c}[\rho(\mathbf{r}, t)]}{\delta \rho\left(\mathbf{r}^{\prime}, t^{\prime}\right)}\right|_{V_{e x t}=0}
$$

has been introduced. Now it is straightforward to get the Dyson-like equation

$$
\begin{aligned}
\chi\left(\mathbf{r}, \mathbf{r}^{\prime}, \omega\right)= & \chi_{0}\left(\mathbf{r}, \mathbf{r}^{\prime}, \omega\right) \\
& +\int d \mathbf{r}_{1} d \mathbf{r}_{2} \chi_{0}\left(\mathbf{r}, \mathbf{r}_{1}, \omega\right) K\left(\mathbf{r}_{1}, \mathbf{r}_{2}, \omega\right) \chi\left(\mathbf{r}_{2}, \mathbf{r}^{\prime}, \omega\right),
\end{aligned}
$$

which has to be solved iteratively and where the kernel $K$ has been introduced as

$$
K\left(\mathbf{r}_{1}, \mathbf{r}_{2}, \omega\right)=\frac{1}{\left|\mathbf{r}_{1}-\mathbf{r}_{2}\right|}+f_{x c}\left(\mathbf{r}_{1}, \mathbf{r}_{2}, \omega\right) .
$$

Equation (5.11) corresponds to Eq. (2.17) of the Introduction. In fact, Eq. (5.11) and Eq. (5.10) can be obtained directly, as discussed in the Introduction, for the case in which the total potential in the time-dependent Hamiltonian is $V_{H}+V_{x c}$. As also pointed out there, due to the density-only dependence of these potentials the $\delta$ functions that appear in the derivation are such that only two-point functions are involved from the beginning. Equation (5.11) is hence the contraction of Eq. (2.20). Of course, the nontrivial point is that the response func- tion $\chi$ calculated from the fictitious noninteracting system using Eq. (5.11) is equal to the true response function of the interacting system.

This scheme provides an exact representation of the full interacting linear density response as

$$
\begin{aligned}
\rho_{1}(\mathbf{r}, \omega) & =\int d \mathbf{r}^{\prime} \chi_{0}\left(\mathbf{r}, \mathbf{r}^{\prime} ; \omega\right) V_{\mathrm{eff}}\left(\mathbf{r}^{\prime}, \omega\right) \\
& \equiv \int d \mathbf{r}^{\prime} \chi\left(\mathbf{r}, \mathbf{r}^{\prime} ; \omega\right) V_{e x t}\left(\mathbf{r}^{\prime}, \omega\right) .
\end{aligned}
$$

In other words, the exact linear density response of an interacting system can be written as the linear density response of a noninteracting system to the effective perturbation $V_{\text {eff }}$. The exact time-dependent exchangecorrelation kernel is of course unknown, and practical calculations must rely on some approximation. The most commonly used one, due to its simplicity, is the adiabatic local-density approximation, also called the timedependent LDA (TDLDA), in which $f_{x c}\left(\mathbf{r}_{1}, \mathbf{r}_{2}, \omega\right)$ is approximated by the ( $\omega$-independent) functional derivative of the LDA exchange-correlation potential:

$$
f_{x c}^{T D L D A}\left(\mathbf{r}_{1}, \mathbf{r}_{2}\right)=\delta\left(\mathbf{r}_{1}-\mathbf{r}_{2}\right) \frac{\partial V_{x c}^{L D A}\left(\rho\left(\mathbf{r}_{1}\right), \mathbf{r}_{1}\right)}{\partial \rho\left(\mathbf{r}_{1}\right)} .
$$

Apart from this approximation for $f_{x c}$, another approximation has to be made in practical calculations: the static Kohn-Sham orbitals and eigenvalues used to construct $\chi_{0}$ are in fact calculated with an approximate exchange-correlation potential $V_{x c}$, typically the same as the one used in ground-state calculations.

Formally inverting Eq. (5.11), one obtains a compact two-point matrix equation,

$$
\chi(\omega)=\left[1-\chi_{0}(\omega) K(\omega)\right]^{-1} \chi_{0}(\omega),
$$

where the problem of finding the excited-state energies of an interacting system (poles of $\chi$ ) has been mapped to searching the values of $\omega$ for which the operator $R(\omega)$ $=1-\chi_{0}(\omega) K(\omega)$ is not invertible. In fact, $\chi$ has poles at the true excitation energies $\omega=\Omega_{j}$, while $\chi_{0}$ has poles at the Kohn-Sham eigenvalue differences. Hence the singularities of $\chi$ must be canceled by the zeros of $R(\omega)$ [and those of $\chi_{0}$ by the zeros of $R^{-1}(\omega)$ ]. The true excitation energies $\Omega_{j}$ can be characterized as those frequencies where the eigenvalues of $R$ vanish. In other words, the energies of the resulting electron-hole excitations will be renormalized with respect to those of the noninteracting electron-hole pairs. This formalism provides a convenient starting point for calculating the excitation spectrum.

Of course, for the practical solution of Eq. (5.14) all points discussed in the Introduction apply. In particular, when adding a finite small imaginary part to the frequency, the two-point equation can be inverted for each frequency in order to obtain the spectrum. Alternatively, Eq. (5.14) can be transformed into an effective eigenvalue problem, which implies that one is now working with four-point quantities. This second procedure is equivalent to solving the generalized four-point eigen- 
value problem ${ }^{4} R(\omega)|\lambda\rangle=0$ (see Appendix, B.2, in particular $\left.{ }^{4} R \equiv \bar{\Pi}^{-1} \equiv\left[1-{ }^{4} \chi_{0} K\right]\right)$ and yields the equation

$$
c_{n_{1} n_{2}}-\sum_{n_{3} n_{4}} c_{n_{3} n_{4}}\left(f_{n_{1}}-f_{n_{2}}\right) \frac{K_{\left(n_{1} n_{2}\right),\left(n_{3} n_{4}\right)}(\omega)}{\omega-\left(\epsilon_{n_{2}}-\epsilon_{n_{1}}\right)}=0 \text {, }
$$

where the kernel $K_{\left(n_{1} n_{2}\right),\left(n_{3} n_{4}\right)}(\omega)$ $=\left\langle\Phi_{n_{1} n_{2}}|K(\omega)| \Phi_{n_{3} n_{4}}\right\rangle$, with $\Phi_{n_{1} n_{2}}(\mathbf{r}):=\psi_{n_{1}}(\mathbf{r}) \psi_{n_{2}}^{*}(\mathbf{r})$. The index $n$ embodies the spin as well as orbital degrees of freedom. ${ }^{38}$

Looking at the Coulomb contribution to $K$, one recognizes the effective exchange interaction between electron and hole (electron-hole exchange), which also appears in the Bethe-Salpeter equation (4.11) (for $\chi$ and $v$ instead of $\bar{P}$ and $\bar{v}$ ). The diagonal element reads

$$
\iint d \mathbf{r}_{1} d \mathbf{r}_{2} \Phi_{n_{1} n_{2}}^{*}\left(\mathbf{r}_{1}\right) \frac{1}{\left|\mathbf{r}_{1}-\mathbf{r}_{2}\right|} \Phi_{n_{1} n_{2}}\left(\mathbf{r}_{2}\right),
$$

whereas the $f_{x c}$ contribution within the simple TDLDA introduces a local and static attractive electron-hole interaction:

$$
\begin{gathered}
\iint d \mathbf{r}_{1} d \mathbf{r}_{2} \Phi_{n_{1} n_{2}}^{*}\left(\mathbf{r}_{1}\right) \delta\left(\mathbf{r}_{1}-\mathbf{r}_{2}\right) \frac{\partial V_{x c}\left(\mathbf{r}_{1}\right)}{\partial \rho} \Phi_{n_{1} n_{2}}\left(\mathbf{r}_{2}\right) \\
=\int d \mathbf{r} \rho_{n_{1}}(\mathbf{r}) \frac{\partial V_{x c}(\mathbf{r})}{\partial \rho} \rho_{n_{2}}(\mathbf{r})
\end{gathered}
$$

One can see the effects of these two different contributions on the calculated optical spectrum of a finite system, e.g., a small cluster: the Coulomb part shifts the independent electron spectrum to high energies, whereas the exchange-correlation brings it partially back (this can to a certain extent be compared with the excitonic corrections computed in the framework of the many-body theory, as will be discussed in Sec. VI). These effects are clearly seen in Fig. 8, where the optical spectrum calculated within TDLDA for three different clusters is reported.

Equation (5.15) can be simplified by an expansion around one particular Kohn-Sham transition $1 \rightarrow 2$, i.e., by calculating the transition energy $\omega=\Omega$ in first-order perturbation theory (single-pole approximation). Following Petersilka et al. (1996) and considering explicitly the spin degrees of freedom, ${ }^{39}$ one obtains from Eq. (5.15)

$$
\Omega \simeq \omega_{12}+\operatorname{Re}\left[K_{1 \uparrow 2 \uparrow, 1 \uparrow 2 \uparrow}\left(\omega_{12}\right) \pm K_{1 \uparrow 2 \downarrow, 1 \uparrow 2 \downarrow}\left(\omega_{12}\right)\right] .
$$

\footnotetext{
${ }^{38}$ The transition-space representation is based on similarities with the quantum-chemistry time-dependent Hartree-Fock approach (Langhoff et al., 1972; Casida, 1995). However, a simple two-point matrix problem has been transformed into a more complex four-point representation. Note also that Eq. (5.15) can be derived from the condition $\operatorname{det}\left(H^{2 p}-E_{\lambda}\right)=0$ with $H^{2 p}$ of the form of Eq. (B20), and $K$ of the form defined in Eq. (2.15) without the term involving $w$.

${ }^{39}$ In the present section we treat spin effects explicitly, as is natural when considering atomic transitions.
}
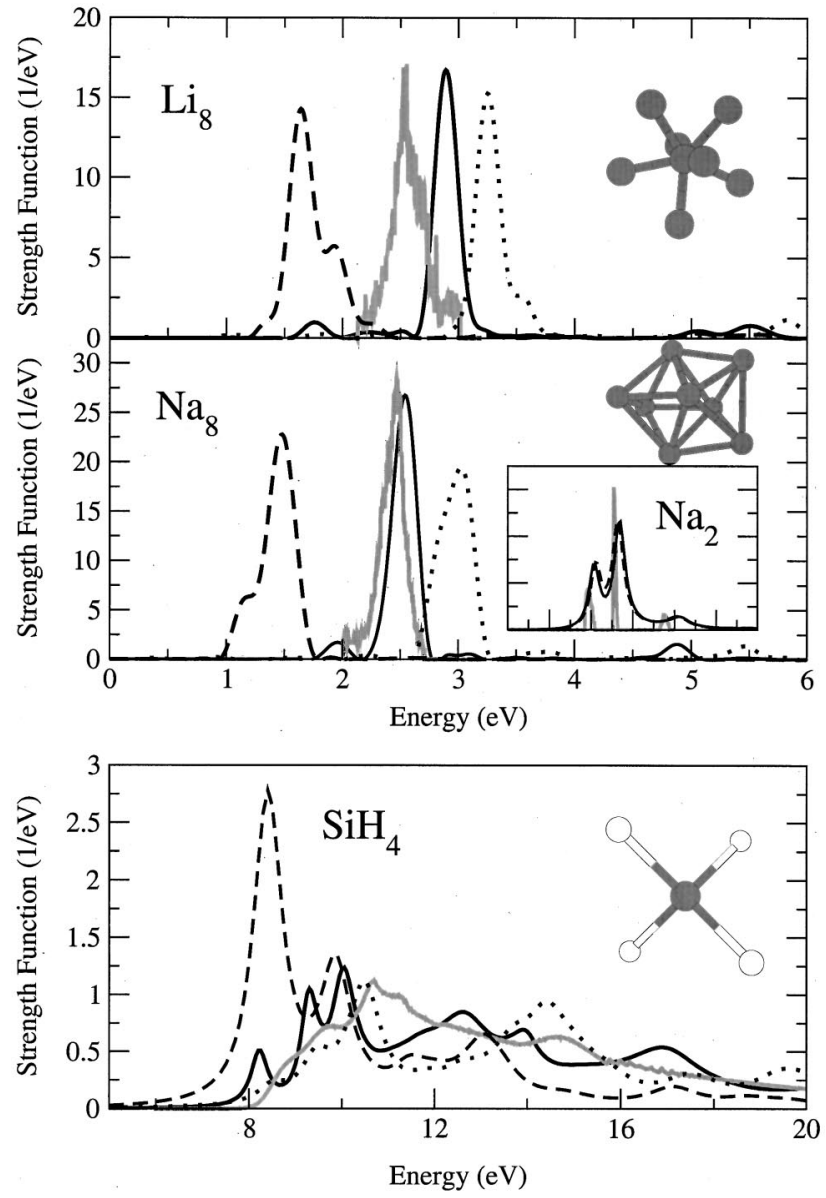

FIG. 8. Calculated optical absorption spectrum (given as the dipolar strength function) for several clusters (Marques et al., 2001), compared to experimental data (Itoh et al., 1986; Wang et al., 1990): solid black line, full TDLDA calculations; dashed line, independent Kohn-Sham particle approximation $\left(\chi_{0}\right)$; dotted line, an RPA calculation, i.e., putting $f_{x c}=0$ in Eq. (5.12); gray line, the experimental results. In the inset of $\mathrm{Na}_{8}$ are the results for the sodium dimer: heavy solid line, full TDLDA calculation; gray line, experimental results (Sinha et al., 1949), but now the dashed line (almost indistinguishable from previous one) is for a calculation using an exact-exchange functional (Marques et al., 2001). As discussed in the text the kernel includes an effective attractive part (electron-hole attraction), which reduces the Coulomb term (electron-hole exchange).

Note that the noninteracting Kohn-Sham response function is diagonal in the spin variables and exhibits poles at the Kohn-Sham energy differences corresponding to noninteracting electron-hole excitations within the same spin space. The mixing of spin channels comes into play simply by the spin-dependent exchange-correlation kernel, and the magnetization response naturally involves spin-flip processes. In order to make more explicit the fact that the approximation in Eq. (5.18) embodies the spin-multiplet structure of the excitation spectrum of otherwise spin-unpolarized ground states, one can rewrite the $f_{x c}$ kernel in terms of the two independent combinations of the spin components of the kernel: $f_{x c}^{1}$ 
$=0.5\left(f_{x c \uparrow \uparrow}+f_{x c \uparrow \downarrow}\right)$ and $f_{x c}^{2}=0.5\left(f_{x c \uparrow \uparrow}-f_{x c \uparrow \downarrow}\right) .{ }^{40}$ Thus the singlet and triplet solutions of Eq. (5.18) are (note again the similarity to the Bethe-Salpeter equation concerning the contribution of $v$ )

$$
\begin{aligned}
\omega_{\text {singlet }}= & \omega_{12}+2 \operatorname{Re} \int d \mathbf{r}_{1} d \mathbf{r}_{2} \Phi_{12}^{*}\left(\mathbf{r}_{1}\right) \\
& \times\left(\frac{1}{\left|\mathbf{r}_{1}-\mathbf{r}_{2}\right|}+f_{x c}^{1}\left(\mathbf{r}_{1}, \mathbf{r}_{2}, \omega_{12}\right)\right) \Phi_{12}\left(\mathbf{r}_{2}\right)
\end{aligned}
$$

and

$$
\begin{aligned}
\omega_{\text {triplet }}= & \omega_{12} \\
& +2 \operatorname{Re} \int d \mathbf{r}_{1} d \mathbf{r}_{2} \Phi_{12}^{*}\left(\mathbf{r}_{1}\right) f_{x c}^{2}\left(\mathbf{r}_{1}, \mathbf{r}_{2}, \omega_{12}\right) \Phi_{12}\left(\mathbf{r}_{2}\right) .
\end{aligned}
$$

Promising results are obtained for the lowest excitation energies of atoms and molecules by using different approximations for $V_{x c}$ and $f_{x c}$ (Petersilka et al., 1996; March et al., 1999; Casida et al., 2000; Petersilka et al., 2000).

Results for finite systems beyond the single-pole approximation which have been particularly discussed are those of TDLDA (Rubio et al., 1996; Yabana and Bertsch, 1996, 1999a, 1999b; Vasiliev et al., 1999, 2002; Casida et al., 2000; Marques et al., 2001) and the results obtained within the OEP scheme ${ }^{41}$ (Görling, 1999; Gross et al., 1996; Petersilka et al., 1996, 2000; Grabo et al., 2000a, 2000b). The latter approach gives an exchange-correlation potential with the correct $-1 / r$ behavior at long distances (Casida and Salahub, 2000). The optimized effective potential yields a uniform shift of the energies of transitions to Rydberg states (Petersilka et al., 2000; Stener et al., 2001) that mimics to some extent the results obtained using the exact $V_{x c}$ available for some atoms (Petersilka et al., 2000). From this work the importance of a good description of the static exchange-correlation potential is clear, as is also the fact that only ground-state quantities are needed in the calculation of excitations. $V_{x c}$ is especially important for the higher atomic excited states, which are almost uniformly shifted from the true excitation energies (the shift can be related to the difference between the ionization potential and the highest occupied orbital, which in exact DFT should be zero). Further developments of time-dependent functionals to treat problems involving excited-state dissociation (Cai et al., 2000; Aryasetiawan et al., 2002a) and autoionization resonances (Stener et al., 2001) are needed. In the latter case, even if the atomic photoionization cross sections are well described using the exact $V_{x c}$ and the TDLDA kernel, this is not

\footnotetext{
${ }^{40}$ The $f_{x c}^{2}$ part of the kernel describes exchange-correlation processes in the Kohn-Sham system related to the linear response of the frequency-dependent magnetization density, whereas $f_{x c}^{1}$ is related to the frequency-dependent density.

${ }^{41}$ The calculations in the OEP scheme handle exchange exactly, and correlations are treated in a local or gradientcorrected adiabatic functional.
}

the case for the autoionization resonances that turn out to be very sensitive to the particular choice of $f_{x c}$.

In extended systems with delocalized states one can directly solve Eq. (5.14) in the complex plane. [The secular Eq. (5.15) becomes a four-point integral equation and is computationally not advantageous]. The zeros of $R$ provide both the excitations as well as the corresponding lifetimes. This technique has been successfully used to describe the plasmon dispersion of a homogeneous electron gas (Tatarczyk et al., 2001). However, the TDLDA has only limited success in describing excitations in extended systems, and most likely both nonlocality in space and time are needed in order to get this renormalization. The successes and failures of TDLDA in finite and infinite systems, respectively, are not yet fully understood. However, it appears that improvements might more easily be found through an improved $V_{x c}$ in the case of finite, and an improved $f_{x c}$ in the case of infinite, systems. In any case, $f_{x c}$ is a quantity of interest which (i) has been discussed in less detail than $V_{x c}$ and (ii) is necessarily more complicated, since it is its functional derivative. Therefore we look more closely at $f_{x c}$ in the following section.

\section{The exchange-correlation kernel $f_{x c}$}

In the previous section we introduced the exchangecorrelation kernel $f_{x c}$ to take into account all the dynamical exchange and correlation effects in the response of a system to an external perturbing potential. An exact representation of $f_{x c}$ in terms of the response functions is obtained directly from Eq. (5.11):

$$
\begin{aligned}
f_{x c}\left(\mathbf{r}, t ; \mathbf{r}^{\prime}, t^{\prime}\right)= & \chi_{0}^{-1}\left(\mathbf{r}, t ; \mathbf{r}^{\prime}, t^{\prime}\right)-\chi^{-1}\left(\mathbf{r}, t ; \mathbf{r}^{\prime}, t^{\prime}\right) \\
& -\frac{\delta\left(t-t^{\prime}\right)}{\left|\mathbf{r}-\mathbf{r}^{\prime}\right|} .
\end{aligned}
$$

Note that for finite systems the frequency-dependent response operators can be noninvertible at isolated frequencies (isolated real poles). However, this is no longer the case for infinite bulk systems. In Appendix A we provide a summary of the known exact properties of $f_{x c}$ that can be used as constraints to build new approximations to the exchange-correlation kernel.

As a consequence of causality, response functions must be zero for $t^{\prime}>t$. Therefore the $f_{x c}$ kernel cannot be symmetric under the exchange of $(\mathbf{r}, t)$ and $\left(\mathbf{r}^{\prime}, t^{\prime}\right)$. Since, on the other hand, $f_{x c}$ is the functional derivative of the exchange-correlation potential, one concludes that either the exact $V_{x c}(\mathbf{r}, t)$ cannot be a functional derivative of the $A_{x c}$ functional (i.e., $f_{x c}$ is not a second functional derivative; see Gross et al., 1996; van Leeuwen, 1998, 1999), or there is a contradiction with the stationary-action principle that we have described as a basic ingredient of time-dependent density-functional theory. This problem applies to all twice-differentiable action functionals defined with respect to the physical time. It appears when applying the variational principle to the action in Eq. (5.1). In fact, from the Runge-Gross (1984) theorem, the wave function is determined up to a 
time-dependent phase factor; this makes the action not uniquely defined unless one fixes this phase factor (in particular it can be chosen such that $A[\Psi]=0$ ). Therefore the action is not a density functional as the density by itself does not fix the phase of the wave function (van Leeuwen, 2001). This apparent contradiction has been formally resolved by van Leeuwen $(1998,2001)$ by defining a new action functional ${ }^{42}$ that properly incorporates causality effects, and it is not made stationary but rather used as a generating function for the density and response functions as in statistical mechanics.

One of the most widely used approximations to timedependent phenomena is the adiabatic LDA or timedependent LDA (TDLDA) in which the static LDA functional is used for the dynamical properties. This means that the $f_{x c}$ kernel is a contact function in time and space [Eq. (5.13)]. Thus $f_{x c}$ is not frequency dependent at all. TDLDA gives rather accurate results for systems with rapidly varying densities such as atoms, surfaces, and clusters. ${ }^{43}$ Gross and Kohn (1985) have presented an extension of the LDA approximation to include dynamical effects in the $f_{x c}$ kernel, and we refer the reader to their work for the details of parametrization. ${ }^{44}$ The idea is to use the homogeneous electron-gas kernel $f_{x c}^{h o m}\left(\left|\mathbf{r}-\mathbf{r}^{\prime}\right|, \omega\right)$ and make the assumption that the linear-induced density is a slowly varying function (as in the traditional static LDA). This amounts to the approximation $f_{x c}\left(\mathbf{r}, \mathbf{r}^{\prime}, \omega\right)$ $=\hat{f}_{x c}^{h o m}(\rho(\mathbf{r}), \omega)$, where $\hat{f}_{x c}^{\text {hom }}$ is the $q=0$ Fourier component of $f_{x c}^{\text {hom }}\left(\rho(\mathbf{r}),\left|\mathbf{r}^{\prime}-\mathbf{r}^{\prime \prime}\right|\right)$.

Other kernels proposed in the literature are the following (spin variables are omitted for simplicity):

- Petersilka, Grossman, and Gross (PGG) kernel. This was derived by Petersilka et al. (1996) in the context of exact exchange time-dependent optimized effective potentials and kernels (Gross et al., 1996; Görling, 1998) (x-TDOEP), and it is equivalent to the so-called Slater approximation in Hartree-Fock calculations (Langhoff et al., 1972). It is a frequency-independent kernel that in real space reads

\footnotetext{
${ }^{42}$ The new action is defined using the time contour method of Keldysh (1965) in which the physical time is parametrized in terms of a parameter called pseudotime. By construction, the response functions obtained as higher-order derivatives of the action functional are symmetrical in the Keldysh pseudotime. A back-transformation to the physical time directly provides the desired causal response functions (see van Leeuwen, 2001 for mathematical details of this technique).

${ }^{43}$ See, for example, (Stott and Zaremba, 1980; Zangwill and Soven, 1980; Dobson et al., 1988; Tsuei et al., 1990; Casida, 1995; Petersilka et al., 1996; Rubio et al., 1996, 1997; Liebsch, 1997; Vasiliev et al., 1999, 2002).

${ }^{44}$ Note that the lower the density the larger the frequency dependence of the kernel. The parametrization can be extended to nonvanishing $q$ and to include spin polarization. However, it fails to reproduce some exact relations, such as the harmonic-potential theorem (Dobson, 1994).
}

$$
f_{x}^{P G G}\left(\mathbf{r}, \mathbf{r}^{\prime}\right)=-\frac{1}{2} \frac{1}{\left|\mathbf{r}-\mathbf{r}^{\prime}\right|} \frac{\left|\sum_{k} f_{k} \psi_{k}(\mathbf{r}) \psi_{k}^{*}\left(\mathbf{r}^{\prime}\right)\right|^{2}}{\rho(\mathbf{r}) \rho\left(\mathbf{r}^{\prime}\right)} .
$$

Note that the general expression of the TDOEP kernel (similar to time-dependent Hartree-Fock) is frequency dependent even at the exchange-only level (Petersilka et al., 1996, 1998), a feature that is not accounted for in the PGG approximation. However for a two-electron system at the exchange-only level the TDOEP and PGG kernels are equivalent, explicitly showing that the dynamical part of the kernel stems from processes involving multiple valence transitions.

- TDOEP-SIC kernel. This is an attempt to improve over both TDLDA and exact exchange (TDOEP) by correcting the TDLDA for the self-interaction error (SIC $=$ self-interaction-corrected). This correction does not affect antiparallel spin contributions. In this case, the kernel reads

$$
\begin{aligned}
f_{x c}^{T D O E P-S I C}\left(\mathbf{r}, \mathbf{r}^{\prime}\right)= & f_{x c}^{T D L D A}\left(\mathbf{r}, \mathbf{r}^{\prime}\right) \\
& -\frac{1}{2} \frac{\sum_{k} f_{k}\left|\psi_{k}(\mathbf{r}) \psi_{k}^{*}\left(\mathbf{r}^{\prime}\right)\right|^{2}}{\rho(\mathbf{r}) \rho\left(\mathbf{r}^{\prime}\right)} \\
& \times\left\{\frac{\partial V_{x c}^{L D A}\left(\rho_{k}(\mathbf{r}), \mathbf{r}\right)}{\partial \rho_{k}\left(\mathbf{r}^{\prime}\right)}+\frac{1}{\left|\mathbf{r}-\mathbf{r}^{\prime}\right|}\right\},
\end{aligned}
$$

where $\rho_{k}$ is the density of the orbital $k$. This expression is exact in the one-electron case and, for more electrons, it corrects the spurious self-interaction of parallel spin, but keeps the antiparallel contributions as in TDLDA. This functional is nevertheless ill defined, since it is not invariant upon a unitary transformation of the Kohn-Sham wave functions.

- BPG kernel. Burke et al. (2002) proposed a hybrid method to improve the excitation spectra of small atoms by combining the previous PGG expression for symmetric spin orientations and the TDLDA for antisymmetric spin orientations. For the case of a homogeneous electron gas it reads $f_{x c}^{B P G}(\mathbf{q})$ $=0.5 *\left[f_{x c, \uparrow \uparrow}^{P G G}(\mathbf{q})+f_{x c, \uparrow}^{T D L D A}(\mathbf{q})\right]$.

- CDOP kernel. Corradini et al. (1998) gave a parametrization of the quantum Monte Carlo data of Moroni et al. (1995) for the homogeneous electron gas that satisfies the theoretically known limits for small and large q. This kernel has an analytical space Fourier transform that simplifies its implementation in standard first-principles techniques.

- RA kernel. Richardson and Ashcroft (1994) proposed a dynamical parametrization of the kernel based on a summation of self-energy and fluctuation terms in the diagrammatic expansion of the polarization function for the homogeneous electron gas. It is constructed to satisfy many known exact conditions (static and dynamic). This parametrization is assumed to be very close to the exact dynamical exchange-correlation kernel of the homogeneous electron gas. A corrected version of the RA parametrization can be found in Lein et al. (2000). 
- TP kernel. Tokatly and Pankratov (2001), based on a many-body diagrammatic expansion, have derived an expression for the $f_{x c}$ kernel in terms of the regular part $\chi_{r}$ of the Kohn-Sham response function $\chi_{0}$ with respect to a given resonance frequency $\omega_{i j}$ :

$$
\begin{array}{r}
f_{x c}\left(\mathbf{r}, \mathbf{r}^{\prime}, \omega_{i j}\right) \\
=\Delta_{i j} \frac{\int d \mathbf{r}_{1} d \mathbf{r}_{2} \chi_{r}^{-1}\left(\mathbf{r}, \mathbf{r}_{1}\right) \Phi_{i j}\left(\mathbf{r}_{1}\right) \Phi_{i j}^{*}\left(\mathbf{r}_{2}\right) \chi_{r}^{-1}\left(\mathbf{r}_{2}, \mathbf{r}^{\prime}\right)}{\left[\int d \mathbf{r}_{1} d \mathbf{r}_{2} \Phi_{i j}^{*}\left(\mathbf{r}_{2}\right) \chi_{r}^{-1}\left(\mathbf{r}_{2}, \mathbf{r}_{1}\right) \Phi_{i j}\left(\mathbf{r}_{1}\right)\right]^{2}},
\end{array}
$$

where $\Delta_{i j}=\left\langle\Phi_{i j}\left|f_{x c}\left(\omega_{i j}\right)\right| \Phi_{i j}\right\rangle\left[\Phi_{i j}\right.$ was defined after Eq. (5.15)]. It is shown that the spatial nonlocality is strongly frequency dependent and diverges for the case of infinite systems at the excitation energies. This result is similar in form to the correction obtained by Gonze and Scheffler (1999) and it is also equivalent to the Görling-Levy perturbation theory (Görling and Levy, 1994).

- RORO kernel. This is a static kernel derived by Reining et al. (2002) from a direct comparison between the time-dependent density-functional and BetheSalpeter equations. In Appendix $\mathrm{C}$ we develop this kernel in greater detail by formally comparing the Bethe-Salpeter equation and TDDFT. The kernel consists of a contribution stemming from the energy shift between Kohn-Sham and $G W$ eigenvalues, and a second one describing the electron-hole interaction. One can absorb the first, positive contribution into an energy shift of the starting $\chi_{0}$. Important excitonic effects are then obtained by using only the static longrange term $\Delta f_{x c}\left(\mathbf{q}, \mathbf{G}, \mathbf{G}^{\prime}\right)=-\delta_{\mathbf{G}, \mathbf{G}^{\prime}} \alpha /|\mathbf{q}+\mathbf{G}|^{2}$.

Some of these kernels have been tested in two limiting cases, for helium and beryllium atoms (Petersilka et al., 2000) and for the correlation energy (Lein et al., 2000) and plasmon dispersion (Tatarczyk et al., 2001) of a homogeneous electron gas. In the case of atoms the detailed form of the $V_{x c}$ potential is the crucial part in getting the absolute position of most excitation energies, and the TDLDA kernel provides reasonable results. The results are marginally improved using more complicated kernels, always keeping the "exact" $V_{x c}$ fixed. In fact the results obtained by Petersilka et al. $(1998,2000)$ for the helium and beryllium atom using different kernels indicate that the influence of the $f_{x c}$ on the calculated spectra is less important than the choice of a good $V_{x c}$ potential. However, this is no longer true for the lower excitation energies of beryllium and for singlettriplet splittings, where the effects coming from $V_{x c}$ cancel. Furthermore, Aryasetiawan et al. (2002a) looked at the singlet excitation of the $\mathrm{H}_{2}$ molecule as a function of the internuclear distance. The results are summarized in Fig. 9, where it can be seen that the simple TDLDA is good for intermediate distances only. The inclusion of spin dependence improves the large-distance results. By comparing with exact results of a simple two-site Hubbard model it was found that, indeed, $f_{x c}$ should be

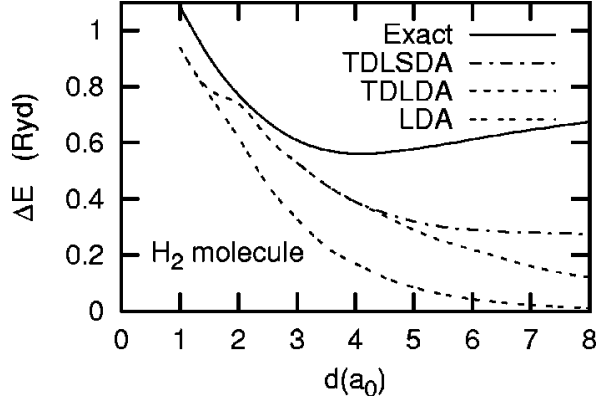

FIG. 9. The excitation energy $\Delta E$ in the hydrogen molecule for the transition from the ${ }^{1} \Sigma_{g}^{+}$state to the ${ }^{1} \Sigma_{u}^{+}$state as a function of the nuclear separation $d$. LDA stands for the eigenvalue difference in LDA, whereas TDLDA and TDSLDA correspond to the calculation of the excitation energy within time-dependent LDA without and with spin polarization, respectively. The full line corresponds to the exact results, provided for comparison (Aryasetiawan et al., 2002).

strongly nonlocal in space and has an important energy dependence. The nonlocality has been clearly shown by Baerends (2001). He proposed an orbital-dependent exchange-correlation potential for the $\mathrm{H}_{2}$ molecule that yields the exact dissociation regime within the KohnSham formalism.

Similar conclusions were obtained by Marques et al. (2001) in a study of the optical absorption spectrum of small sodium and silicon hydrogenated clusters. ${ }^{45}$ Moreover, it is observed that exchange-correlation kernels fitted to reproduce atomic properties perform poorly in the case of an extended electron gas, due mainly to incorrect behavior at long wavelengths. This limit is well reproduced in the TDLDA but not in the PGG kernel. Furthermore, the static CDOP kernel gives results of similar quality to those of the more elaborated dynamical RA kernel. This indicates that the frequency dependence of the kernel is of little importance in providing good total correlation energies and plasmon dispersion. Note that TDLDA does not perform too badly in this last case (Larson et al., 1996).

Finally, Reining et al. (2002) have tested the abovementioned long-range contribution to the static RORO kernel by performing a TDDFT calculation for bulk silicon in the following way. First, they determined the LDA electronic structure. Second, they constructed $\chi_{0}$, but with the eigenvalues shifted to the $G W$ ones, in order to simulate the first part of the kernel as explained above. Third, they used $\Delta f_{x c}\left(\mathbf{r}, \mathbf{r}^{\prime}\right)=-\alpha /\left(4 \pi\left|\mathbf{r}-\mathbf{r}^{\prime}\right|\right)$, with the empirical value $\alpha=0.2$. The result of the TDDFT-RORO calculation is shown in Fig. 10. The dots are the experimental results for the absorption spectrum measured by Lautenschlager et al. (1987). The dotted and dot-dashed curves are the results of a RPA and a

\footnotetext{
${ }^{45}$ All the results for the optical spectrum of small Na clusters are very similar, regardless of the exchange-correlation potential used. In contrast, hydrogenated silicon clusters show that a much better agreement with diffusion quantum Monte Carlo calculations (Grossman et al., 2001; Porter et al., 2001) and with experiments can be obtained when the exact exchange potential is used.
} 


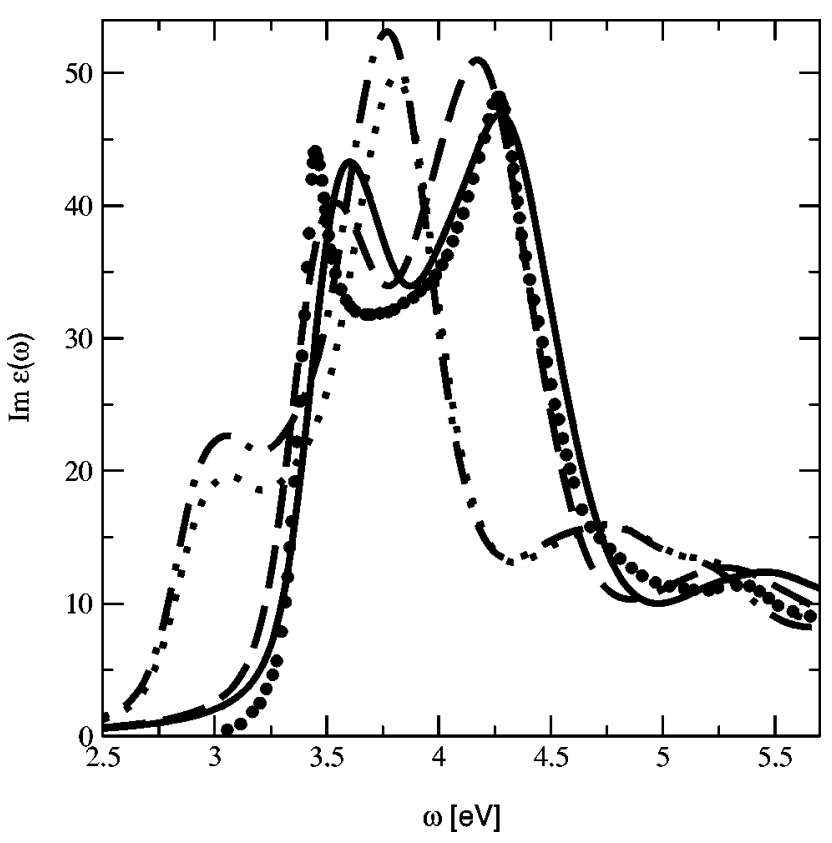

FIG. 10. Optical spectrum $\left[\operatorname{Im}\left(\varepsilon_{M}\right)\right]$ for silicon: $\bullet$, experiment (Lautenschlager et al., 1987); dotted curve, RPA; dot-dashed curve, TDLDA; long-dashed curve, Bethe-Salpeter equation; continuous curve, TDDFT using RORO kernel (Reining et al., 2002; see text for details).

TDLDA calculation. The well-known discrepancies with experiment are found. The solid curve is the result of the approximate TDDFT-RORO calculation, visibly in close agreement with the Bethe-Salpeter result (dashed) and with experiment.

Similarly good agreement has been found for the optical spectra of other semiconductors (Botti et al., 2002). It turns out that this static long-range contribution to the kernel is sufficient to reproduce strong continuum excitonic effects in semiconductors. Promising results were also obtained for the optical spectrum of some simple semiconductors by Kim et al. (2002) using the exact exchange Kohn-Sham formalism together with a TDLDA $f_{x c}$, and by de Boeij et al. (2001) using a polarizationdependent functional within the current-density functional proposed by Vignale and Kohn (1996). This procedure shows the influence of macroscopic electric fields in the response function. However, none of these approaches has up to now managed to describe bound excitons, and one should certainly at least go beyond the simple $-1 / q^{2}$ approximation for the RORO kernel in order to describe such effects.

One can conclude that the use of TDDFT for the calculation of neutral excitations is promising: in the lowenergy range of the absorption spectra of clusters, TDDFT, even in the adiabatic local-density approximation, significantly corrects peak positions with respect to those found when Kohn-Sham eigenvalue differences are interpreted as excitation energies. Typically, the error in excitation energy reduces from an amount of the order of $50 \%$ to an order of magnitude of $10 \%$ (often, the main correction comes from $v$ or $\bar{v}$, i.e., the variation of the Hartree potential). Also, valence plasmons in sol- ids are well described in TDLDA for small, momentum exchange, yielding plasmon frequencies that agree with the experimental ones within $0.5-2 \mathrm{eV}$, and good line shapes. However, the optical absorption spectra of solids are not improved by TDLDA with respect to the sum of Kohn-Sham transitions (see also Sec. VI.D.1). More precisely, calculations performed on many different semiconductors (Gavrilenko and Bechstedt, 1996; Kootstra et al., 2000) demonstrate that TDLDA spectra are, on average, redshifted by about $40 \%$ of the gap. Even when the gap is corrected with a scissor operator [as is commonly done for LDA calculations (Levine and Allan, 1989)], significant discrepancies with experiment remain: typically, in zinc-blende semiconductors the strength of the $E_{1}$ peak is underestimated by $0-40 \%$, that of $E_{2}$ is overestimated by $30-100 \%$, and the absorption onset is overestimated by $0.5 \mathrm{eV}$ (e.g., in GaSb, InAs, $\mathrm{ZnTe}$ ) or even by $0.8 \mathrm{eV}$ (in CdTe and InSb). It is clear that better exchange-correlation potentials $V_{x c}$ and kernels $f_{x c}$ must be found in order to make TDDFT become the approach for the calculation of absorption spectra, just as static DFT is today the predominant method for $a b$ initio calculations of ground-state properties. In fact, $f_{x c}$ must be a strongly nonlocal functional of the density, and, in principle, frequency dependent. It seems to be crucial to include in $f_{x c}$ at least a contribution going as $-1 / q^{2}$ for $q \rightarrow 0$, in order to reproduce continuum excitonic effects in the absorption spectra of infinite systems. See also Appendix C.

\section{TDDFT VERSUS BETHE-SALPETER}

TDDFT and the Green's-function approach represent two complementary ways to calculate a dynamical dielectric matrix and the spectra derived from it. The ultimate goal should of course be either to decide whether one of the two approaches is clearly superior in a given situation, or whether it is possible to combine the advantages of both in order to design a reliable and efficient way to calculate electronic spectra for a broad range of applications. It is therefore useful to compare the two approaches on different levels: from the mathematical point of view, by trying to work out which effects the different approximations might cause, and by comparing results for different systems and types of spectroscopies. It should not be forgotten that in principle both approaches are exact, and failures can only be explained by the unavoidable approximations, or by an application that is in principle not adequate for a particular problem (as in the case of static DFT and the interpretation of Kohn-Sham eigenvalues as electron addition or removal energies).

\section{A. The equations}

Let us first examine the structure of the equations. It should be kept in mind that, whether absorption or loss spectra are to be calculated, and whether TDDFT or the Bethe-Salpeter approach is used, the equation to be solved is of the form $S=P_{I Q P}+P_{I Q P} K S$ [see Eqs. (4.10) and (5.12)]. Here $S$ may be either $\chi$ or $\bar{P}$ (describing loss 
and absorption spectra, respectively), depending on whether the kernel $K$ contains the full Coulomb interaction $v$ or the truncated $\bar{v}$. Moreover, $K$ has a contribution $F$ containing the exchange-correlation effects. There are two important differences between the TDDFT and the Bethe-Salpeter approaches. First, $P_{I Q P}$ is either the Kohn-Sham $\left(\chi_{0}\right)$ or the quasiparticle independent-particle response. The second difference concerns $F$. In particular, since the Bethe-Salpeter approach derives from Green's functions (i.e., densitymatrix-like quantities), it naturally leads to a four-point function $F$, whereas the TDDFT equation, which is dealing with density-only potentials, can be contracted and yields a two-point equation, since the delta functions in the $v$ or $\bar{v}$ part of the kernel [Eq. (4.10)] are such that one can contract Eq. (4.9) and get the two-point $S$ without solving the four-point equation first [this corresponds to using the $f_{x c}$ defined in the previous section, Eq. (5.10)].

The Bethe-Salpeter equation with the full kernel [Eq. (4.10)] cannot be contracted, because of the fact that the $\delta$ functions of the exchange-correlation part are connecting different indices from those of the local field part. If one supposes for a moment that the self-energy could be approximated by the DFT exchange-correlation potential, for the calculation of one-quasiparticle eigenvalues but also for the functional derivative leading to the kernel, one would obtain

$$
\begin{aligned}
\delta\left(\mathbf{r}_{1}\right. & \left.-\mathbf{r}_{2}\right) \frac{\delta V_{x c}\left(\mathbf{r}_{1}\right)}{\delta G\left(\mathbf{r}_{3}, \mathbf{r}_{4}\right)} \\
& =\delta\left(\mathbf{r}_{1}-\mathbf{r}_{2}\right) \int d \mathbf{r}_{5} \frac{\delta V_{x c}\left(\mathbf{r}_{1}\right)}{\delta \rho\left(\mathbf{r}_{5}\right)} \frac{\delta \rho\left(\mathbf{r}_{5}\right)}{\delta G\left(\mathbf{r}_{3}, \mathbf{r}_{4}\right)} \\
& =f_{x c}\left(\mathbf{r}_{1}, \mathbf{r}_{3}\right) \delta\left(\mathbf{r}_{1}-\mathbf{r}_{2}\right) \delta\left(\mathbf{r}_{3}-\mathbf{r}_{4}\right),
\end{aligned}
$$

the same structure of connecting $\delta$ functions as in the Hartree (i.e., $v$ ) part. Again, one can contract the equation and obtain the TDDFT two-point form. This is of course true for any form of the exchange-correlation potential that is purely density dependent and local, whereas for the nonlocal and Green's-functiondependent potentials of many-body perturbation theory the equations are necessarily four-point ones. Of course this reasoning should not cause the misunderstanding that TDDFT is an approximation to the Bethe-Salpeter equation; besides the fact that it is a true and in principle exact alternative, there are also subtleties linked to the use of time-ordered and retarded quantities which prohibit an easy switching between the two. However, the discussion of the structure of the equations still remains valid.

Equations (4.9) and (5.11) are not the only way to write the Bethe-Salpeter and TDDFT schemes. In fact, in the previous sections we have already seen that it is often convenient to transform these equations to an effective eigenvalue equation, namely, Eq. (4.11) for the Bethe-Salpeter equation, and Eq. (5.15), multiplied by $\left(\omega-\omega_{j k}\right)$, for TDDFT. It should be pointed out that in this case both equations have become four-point equa- tions, because they have been obtained by a basis transformation to a basis of pairs of occupied and empty states. The two equations now look exactly identical, the only differences being the meaning of the one(quasi)particle eigenvalues and the content of the kernel. Clearly, this way of rewriting the equations is convenient if (a) the single diagonalization to be performed is less onerous than the inversion of $\chi$ for many frequencies (which necessarily assumes that the kernel does not depend on frequency), and/or (b) if the basis of pairs of states which must be considered is smaller than a realspace (or reciprocal-space) basis. This is naturally true for the Bethe-Salpeter equation, where those basis sets would also be quadratic. In the case of TDDFT, the quadratic basis of states must be compared to the linear basis in real or reciprocal space. In that case, the choice of the four-point form can only be convenient for small finite systems with well-spaced energy levels and in which only a limited part of the spectrum is needed. A larger energy range would require a higher number of states, increasing considerably the computational effort in this four-point approach. This explains why one finds this form mostly in applications of quantum chemists.

Writing the equation in the basis of transitions has the additional drawback that the full Hamiltonian [Eq. (B20)], i.e., that including resonant, antiresonant, and coupling contributions, is non-Hermitian. It has been shown that this problem can be avoided by transforming the equation into a simple quadratic one of the size of the resonant contribution only (Casida, 1995; Bauernschmitt and Ahlrichs, 1996):

$$
(R-C)(R+C) a=\omega^{2} a,
$$

where $R$ and $C$ are the resonant and coupling matrices, respectively, and $a$ is a linear combination of the two corresponding parts of the full eigenvector $A_{\lambda}$ (see Appendix B.2 for details). In the case of TDDFT and for real wave functions, $R_{v c v^{\prime} c^{\prime}}=\omega_{v c} \delta_{v v^{\prime}} \delta_{c c^{\prime}}+K_{v c v^{\prime} c^{\prime}}$ and $C_{v c v^{\prime} c^{\prime}}=K_{v c v^{\prime} c^{\prime}}$. Then $R-C$ is diagonal, and the quadratic equation can be simplified; it reads (in its symmetrized form)

$\left[\omega_{j k}^{2} \delta_{j l} \delta_{k m}+2 \sqrt{f_{j k} \omega_{j k}} K_{j k, l m}(\omega) \sqrt{f_{l m} \omega_{l m}}\right] c_{l m}=\omega^{2} c_{j k}$,

where all the spin degrees are embodied in the $i, j$ indexes, and $f_{j k}=f_{j}-f_{k}$. Here one is explicitly taking into account transitions of the type $l \rightarrow m$ where $l$ is occupied and $m$ is unoccupied. In the Bethe-Salpeter equation, the electron-hole attraction term does not show the symmetry $C_{v c v^{\prime} c^{\prime}}=K_{v c v^{\prime} c^{\prime}}$, and Eq. (6.2) cannot be simplified. This can be a considerable problem in applications like the calculation of electron-energy-loss spectra, where the resonant-antiresonant coupling cannot be neglected, since in that case the non-Hermitian Hamiltonian [or, equivalently, Eq. (6.2)], do not allow the application of fast inversion techniques like the Haydock recursion method (Haydock, 1980).

The third main representation of the TDDFT equation is the explicitly time-dependent one. In fact, the 
spectra we are interested in are due to the response of the system to a time-dependent external field. It is hence possible to extract the desired information from a calculation of the time evolution of the density when such a perturbation is applied to the system. This corresponds to going one step back in the derivation of the equations, as was done, for example, in the introduction to this review. Since in TDDFT the density is easily constructed from the time-dependent wave functions, it is sufficient to solve the time-dependent Schrödinger equation for $\psi$ and construct the density at every step. This is particularly easy when approximations for the potential are chosen that do not contain memory effects, which means that not only are the requirements of computer time modest but the storage requirements are low. This method thus uses a direct solution of the timedependent single-electron Schrödinger equation for the occupied states,

$$
i \frac{\partial \psi_{i}(\mathbf{r}, t)}{\partial t}=H_{K S}(t) \psi_{i}(\mathbf{r}, t) \quad(\mathrm{i}=1 \cdots \text { occ. }),
$$

where $H_{K S}$ is the Kohn-Sham Hamiltonian and $\rho$ is the time-dependent density $\rho(\mathbf{r}, t)=\sum_{i=1}^{o c c} \psi_{i}^{*}(\mathbf{r}, t) \psi_{i}(\mathbf{r}, t)$. The solution of this equation relies on very simple sparsematrix-vector multiplications and on a numerical implementation of the unitary time evolution operator (Yabana and Bertsch, 1996, 1999a, 1999b; Bertsch, Iwata, et al., 2000; Bertsch, Rubio, and Yabana, 2000). Hence, for example, in the case of the TDLDA approximation for a cluster, the solution of the matrix equation (5.15) with a given kernel $f_{x c}$ is equivalent to propagating the equation above for some femtoseconds (the number depending on the accuracy in energy for the spectrum; Yabana and Bertsch, 1996, 1999a) with a given (now timedependent) $V_{x c}$. The real-time method has two major advantages: it is nonperturbative and therefore allows nonlinear effects of large fields to be calculated with the same effort, and it uses the same energy functional for the dynamical calculation as for the static calculation used to prepare the ground state, i.e., the potential and its density variation are automatically consistent, without the need to calculate the kernel explicitly.

In principle, the Bethe-Salpeter equation can also be put into the same form, since the time-dependent density can be obtained as the diagonal of the timedependent Green's function (Kadanoff and Baym, 1962). In that case, the time evolution equation for the Green's function must be solved. This has already been proposed by Kwong and Bonitz (2000) and applied to the model case of plasma oscillations, including damping effects on a correlated electron gas. The technique is similar in nature to solving the time-dependent HartreeFock equation (Langhoff et al., 1972); in principle, using the proper self-energy as in the $G W$ approximation, the time evolution propagation is equivalent to solving the Bethe-Salpeter equation. An application to real systems has never been tried, although the idea seems appealing, since instead of solving a four-point equation one need only perform a sequence of (two-point) matrix-matrix multiplications, giving the action of $\Sigma$ on $G$. However, since $G$ is nonlocal, and since the time dependence of $\Sigma$ (and hence memory effects) cannot be neglected, it is not obvious whether such an application would be feasible, not least because of storage requirements.

\section{B. The limit of isolated electrons}

Before discussing results, it is useful to make a few remarks using a model system and/or simplified equations. To this end, working with exchange only already allows one to understand many things. Note that, at least for finite systems, exchange only should be a much better approximation to reality for the two-particle excited states than for, say, photoemission, since screening is less important for a neutral than for a charged excitation.

This exchange-only approximation has the advantage that one can work with relatively simple (explicitly known) exchange operators, and with the DFT exactexchange potential (Gross et al., 1996). This latter potential (assuming from now on all wave functions to be real for simplicity) is given by

$$
\begin{aligned}
V_{x}(\mathbf{r})= & 2 \sum_{v} \sum_{c} \int d \mathbf{r}^{\prime} \int d \mathbf{r}_{1} \int d \mathbf{r}_{2} \psi_{v}\left(\mathbf{r}_{1}\right) \\
& \left.\times \sum_{v^{\prime}} \frac{\psi_{v^{\prime}}\left(\mathbf{r}_{1}\right) \psi_{v^{\prime}}\left(\mathbf{r}_{2}\right)}{\left|\mathbf{r}_{1}-\mathbf{r}_{2}\right|} \psi_{c}\left(\mathbf{r}_{2}\right) \frac{\psi_{c}\left(\mathbf{r}^{\prime}\right) \psi_{v}\left(\mathbf{r}^{\prime}\right)}{\boldsymbol{\epsilon}_{v}-\boldsymbol{\epsilon}_{c}}\right) \\
& \times \chi_{0}^{-1}\left(\mathbf{r}, \mathbf{r}^{\prime}\right),
\end{aligned}
$$

where $\chi_{0}\left(\mathbf{r}, \mathbf{r}^{\prime}\right)$ is the static independent particle response function. To simplify the following considerations further, let us suppose that the system has only one electron; $V_{x}$ is then (Taut, 1992)

$$
V_{x}([\rho], \mathbf{r})=-\int d \mathbf{r}^{\prime} \frac{\rho\left(\mathbf{r}^{\prime}\right)}{\left|\mathbf{r}-\mathbf{r}^{\prime}\right|} .
$$

The "quasiparticle correction" $\left\langle\psi_{v}\left|\Sigma_{x}\right| \psi_{v}\right\rangle-\left\langle\psi_{v}\left|V_{x}\right| \psi_{v}\right\rangle$ is hence zero for the occupied state, and for the lowest unoccupied state it becomes

$$
\begin{aligned}
\Delta^{Q P}= & \left\langle\psi_{c}\left|\Sigma_{x}\right| \psi_{c}\right\rangle-\left\langle\psi_{c}\left|V_{x}\right| \psi_{c}\right\rangle \\
= & -\int d \mathbf{r} \int d \mathbf{r}^{\prime} \frac{\psi_{c}(\mathbf{r}) \psi_{v}(\mathbf{r}) \psi_{v}\left(\mathbf{r}^{\prime}\right) \psi_{c}(\mathbf{r})}{\left|\mathbf{r}-\mathbf{r}^{\prime}\right|} \\
& +\int d \mathbf{r} \int d \mathbf{r}^{\prime} \frac{\left|\psi_{c}(\mathbf{r})\right|^{2}\left|\psi_{v}\left(\mathbf{r}^{\prime}\right)\right|^{2}}{\left|\mathbf{r}-\mathbf{r}^{\prime}\right|} .
\end{aligned}
$$

Now, when considering only one occupied and the lowest empty state (single-pole approximation), the effect of the Hartree kernel and the electron-hole interaction is a change of the optical gap by the matrix elements of the Coulomb interaction $v$ and the electron-hole interaction (in this case also unscreened) taken with respect to the pair $(v, c)$. The latter contribution is

$$
\Delta^{e-h}=-\int d \mathbf{r} \int d \mathbf{r}^{\prime} \frac{\left|\psi_{c}(\mathbf{r})\right|^{2}\left|\psi_{v}\left(\mathbf{r}^{\prime}\right)\right|^{2}}{\left|\mathbf{r}-\mathbf{r}^{\prime}\right|},
$$

which exactly cancels the second term $-\left\langle\psi_{c}\left|V_{x}\right| \psi_{c}\right\rangle$ of the quasiparticle correction [Eq. (6.7)], whereas the electron-hole exchange interaction (the Hartree kernel) 
cancels the first term of Eq. (6.7), $\left\langle\psi_{c}\left|\Sigma_{x}\right| \psi_{c}\right\rangle$. On the other hand, in the TDDFT approach, the total correction is simply the matrix element

$$
\begin{aligned}
\Delta^{T D D F T}= & \int d \mathbf{r} \int d \mathbf{r}^{\prime} \psi_{c}^{*}(\mathbf{r}) \psi_{v}(\mathbf{r}) \\
& \times\left(\frac{1}{\left|\mathbf{r}-\mathbf{r}^{\prime}\right|}+f_{x}\left(\mathbf{r}, \mathbf{r}^{\prime}\right)\right) \psi_{v}^{*}\left(\mathbf{r}^{\prime}\right) \psi_{c}\left(\mathbf{r}^{\prime}\right),
\end{aligned}
$$

which, using $f_{x}\left(\mathbf{r}, \mathbf{r}^{\prime}\right)=\delta V_{x} / \delta \rho\left(\mathbf{r}^{\prime}\right)=-1 /\left|\mathbf{r}-\mathbf{r}^{\prime}\right|$ with the above $V_{x}$ [Eq. (6.5)], yields exactly the same result. Hence in both approaches the net correction to the eigenvalue gap is zero, as it should be. This is of course due to the fact that in this simple model the valence exchange term is simply the self-interaction correction, and it completely cancels the Hartree term. Nevertheless, it is instructive to study this case, since it illustrates how the matrix elements of $f_{x}$ simulate the sum of selfenergy corrections and the electron-hole interaction. Moreover, even in more realistic systems, such as molecules or clusters, the self-interaction contributions can be very strong. In particular, for other approximate kernels that do not treat exchange exactly, this cancellation can be incomplete and the one-electron limit may be wrong.

If one admits more conduction states, it turns out again that in this exchange-only TDDFT all off-diagonal elements are zero, since the total kernel $v+f_{x}$ is zero. This is not true for the Bethe-Salpeter approach, where the contributions to the kernel coming from the Hartree part and the Fock part do not cancel, since they connect different indices. Therefore, in order to see whether the one-electron limit is correct, one should check the contribution of the off-diagonal elements. This is most easily done in perturbation theory. Going beyond the diagonal (first-order) approximation of the Bethe-Salpeter equation yields a second-order correction to the excitation energy which happens to be

$$
E_{\lambda}^{(2)}=\sum_{c^{\prime} \neq c} \frac{\left|-\left\langle\psi_{c}\left|\Sigma_{x}\right| \psi_{c^{\prime}}\right\rangle-\left\langle\psi_{c}\left|V_{H}\right| \psi_{c^{\prime}}\right\rangle\right|^{2}}{\left(\epsilon_{c}^{Q P}-\epsilon_{c^{\prime}}^{Q P}\right)},
$$

where the first term in the numerator is the matrix element between particle-hole pairs $(v, c)$ and $\left(v, c^{\prime}\right)$ of the electron-hole exchange, and the second term in the numerator is exactly equal to the matrix element of the direct electron-hole interaction. In the denominator, one has quasiparticle eigenvalues of empty states. This suggests a deviation from the correct one-electron result, by a shift $-\left|E_{\lambda}^{(2)}\right|$ to lower excitation energies. However, there are further corrections, due to the fact that the above formulas have been obtained (as is usually the case in a $G W$ calculation) using Kohn-Sham wave functions, i.e., performing the $G W$ calculation in first-order perturbation theory. One has in fact to calculate the correction obtained by going to second order in the quasiparticle eigenvalues and taking into account the corresponding correction to the wave functions in the evaluation of the matrix elements of the exciton Hamiltonian. It turns out that the change in eigenvalues adds another $-\left|E_{\lambda}^{(2)}\right|$ to the excitation energy, whereas the update of the wave functions shifts the optical gap by $+2\left|E_{\lambda}^{(2)}\right|$. Moreover, in this model system the resonantantiresonant coupling terms are exactly vanishing. The final result is hence again correct.

This suggests the following observation concerning TDDFT in the exact-exchange approximation and Bethe-Salpeter calculations for very small systems: It is reasonable to neglect off-diagonal elements in the TDDFT equation (single-pole approximation), as well as in the Bethe-Salpeter method, if Kohn-Sham wave functions are used and $G W$ corrections are calculated to first order. However, if off-diagonal elements are included in the Bethe-Salpeter equation, it may be necessary to go beyond first-order perturbation theory in the $G W$ calculation. On the other hand, resonantantiresonant coupling terms can be neglected for those systems.

One can also examine in detail whether the predictions made on the basis of these model calculations are valid. This can be deduced from the results of calculations on $\mathrm{SiH}_{4}$ done by Rohlfing and Louie (2000), in which Bethe-Salpeter results are compared to quantum Monte Carlo results (Grossman et al., 2001) and to experiment. First, Table III of Rohlfing and Louie (2000) confirms that the resonant-antiresonant coupling is indeed completely negligible. Second, the same table confirms that the inclusion of off-diagonal elements of the resonant electron-hole Hamiltonian lowers the excitation energy (by $0.41 \mathrm{eV}$ for the first singlet transition). Then, Table II shows an increase of the transition energy by $0.67 \mathrm{eV}$ due to the off-diagonal elements of $\Sigma$ $-V_{x c}$. This is in agreement with the above qualitative predictions concerning the cancellation of both effects. It suggests, moreover, as also pointed out by Rohlfing and Louie (2000), that most of what is going on can be understood on the basis of Hartree-Fock, selfinteraction corrections and long-range potentials. In fact, the (not self-consistent) time-dependent HartreeFock results presented in the second column of Table II are clearly in acceptable agreement with the corresponding Bethe-Salpeter results.

On the other hand, these findings should not be extrapolated to larger systems: in a solid, in fact, $\Sigma$ preserves the crystal symmetry and can therefore not mix functions of different $k$ points. The exciton Hamiltonian, on the contrary, does strongly mix those transitions which are close in energy and which often come from the same band but different $k$ points. Normally, one will find that quasiparticle wave functions are close to the Kohn-Sham ones, as has been pointed out by Hybertsen and Louie (1986), whereas a strong excitonic effect that is entirely due to the mixing of transitions at different $k$ points drastically alters the absorption spectrum. Note that in a solid first-order perturbation theory with respect to the electron-hole interaction yields a vanishing shift of transition energies, and in fact the modifications in the spectra (even those appearing as shifts of peak positions) are essentially due to the mixing of transition matrix elements. As pointed out in Sec. IV.B, the joint density of states remains virtually un- 
changed (Rohlfing and Louie, 1998b; Benedict and Shirley, 1999). Of course, this question about wave functions does not arise in the TDDFT approach: since the latter never pass through electron addition and removal energies, only the Kohn-Sham wave functions resulting from the ground-state calculation are needed at all steps.

To go further, one can now extend the model and consider the case of more than one valence orbital, but in the approximation that these orbitals are nonoverlapping. Again, one can start from the exact-exchange potential. Because the orbitals are nonoverlapping, one has $v=v^{\prime}$ in Eq. (6.5), which yields

$$
\begin{aligned}
V_{x}(\mathbf{r})= & 2 \sum_{v} \sum_{c} \int d \mathbf{r}^{\prime} \int d \mathbf{r}_{1} \int d \mathbf{r}_{2} \\
& \left.\times \frac{\left|\psi_{v}\left(\mathbf{r}_{1}\right)\right|^{2}}{\left|\mathbf{r}_{1}-\mathbf{r}_{2}\right|} \frac{\psi_{v^{\prime}}\left(\mathbf{r}_{2}\right) \psi_{c}\left(\mathbf{r}_{2}\right) \psi_{c}\left(\mathbf{r}^{\prime}\right) \psi_{v}\left(\mathbf{r}^{\prime}\right)}{\epsilon_{v}-\boldsymbol{\epsilon}_{c}}\right) \\
& \times \chi_{0}^{-1}\left(\mathbf{r}^{\prime}, \mathbf{r}\right) .
\end{aligned}
$$

Now, since the valence orbitals are nonoverlapping, $\chi_{0}\left(\mathbf{r}, \mathbf{r}^{\prime}\right)$ is of block form in $\left(\mathbf{r}, \mathbf{r}^{\prime}\right)$, each of these blocks being given by a region $R_{l}$ which contains both $\mathbf{r}$ and $\mathbf{r}^{\prime}$ and having contributions from one valence orbital $v_{l}$ only. We call these contributions $\chi_{0 v_{l}}\left(\mathbf{r}, \mathbf{r}^{\prime}\right)$. The inverse of $\chi_{0}$ is then given by the inverse of each block, and the above formula yields

$$
\begin{aligned}
V_{x}(\mathbf{r})= & -\sum_{v_{l}, v_{l^{\prime}}} \int d \mathbf{r}^{\prime} d \mathbf{r}_{1} d \mathbf{r}_{2} \\
& \times \frac{\left|\psi_{v_{l}}\left(\mathbf{r}_{1}\right)\right|^{2}}{\left|\mathbf{r}_{1}-\mathbf{r}_{2}\right|} \chi_{0 v_{l}}\left(\mathbf{r}_{2}, \mathbf{r}^{\prime}\right) \chi_{0 v_{l^{\prime}}}^{-1}\left(\mathbf{r}^{\prime}, \mathbf{r}\right) .
\end{aligned}
$$

The integral in $\mathbf{r}^{\prime}$ has nonvanishing contributions of the form $\delta\left(\mathbf{r}_{2}-\mathbf{r}\right)$ when $l=l^{\prime}$ and $\mathbf{r}$ is in Re, and one gets

$$
V_{x}(\mathbf{r})=-\int_{R_{\mathbf{r}}} d \mathbf{r}_{1} \frac{\rho\left(\mathbf{r}_{1}\right)}{\left|\mathbf{r}_{1}-\mathbf{r}\right|} .
$$

The integration is limited to the region $R_{\mathbf{r}}$ where $\mathbf{r}$ is situated. The total quasiparticle correction stemming from both occupied and empty states is hence

$$
\begin{aligned}
\Delta^{Q P}= & \left\langle\psi_{c}\left|\Sigma_{x}\right| \psi_{c}\right\rangle \\
& -\left\langle\psi_{v}\left|\Sigma_{x}\right| \psi_{v}\right\rangle-\left\langle\psi_{c}\left|V_{x}\right| \psi_{c}\right\rangle+\left\langle\psi_{v}\left|V_{x}\right| \psi_{v}\right\rangle, \\
\Delta^{Q P}= & \left\langle\psi_{c}\left|\Sigma_{x}\right| \psi_{c}\right\rangle+\int d \mathbf{r} \int_{R_{\mathbf{r}}} d \mathbf{r}_{1} \frac{\rho_{c}(\mathbf{r}) \rho\left(\mathbf{r}_{1}\right)}{\left|\mathbf{r}_{1}-\mathbf{r}\right|} \\
& +\sum_{v^{\prime}} \int d \mathbf{r} \int d \mathbf{r}_{1} \frac{\psi_{v}(\mathbf{r}) \psi_{v^{\prime}}(\mathbf{r}) \psi_{v^{\prime}}\left(\mathbf{r}^{\prime}\right) \psi_{v}\left(\mathbf{r}^{\prime}\right)}{\left|\mathbf{r}-\mathbf{r}^{\prime}\right|} \\
& -\int d \mathbf{r} \int_{R_{\mathbf{r}}} d \mathbf{r}_{1} \frac{\rho_{v}(\mathbf{r}) \rho\left(\mathbf{r}_{1}\right)}{\left|\mathbf{r}_{1}-\mathbf{r}\right|} .
\end{aligned}
$$

In the diagonal approximation to the Bethe-Salpeter equation, the electron-hole exchange and the electronhole interaction give, respectively, the contributions

$$
\Delta_{x}^{e-h}=+\int d \mathbf{r} \int d \mathbf{r}^{\prime} \psi_{c}(\mathbf{r}) \psi_{v}(\mathbf{r}) \psi_{v}\left(\mathbf{r}^{\prime}\right) \psi_{c}\left(\mathbf{r}^{\prime}\right) /\left|\mathbf{r}-\mathbf{r}^{\prime}\right|
$$

and

$$
\Delta^{e-h}=-\int d \mathbf{r} \int d \mathbf{r}^{\prime}\left|\psi_{c}(\mathbf{r})\right|^{2}\left|\psi_{v}\left(\mathbf{r}^{\prime}\right)\right|^{2} /\left|\mathbf{r}-\mathbf{r}^{\prime}\right|,
$$

as in the simpler model above. Adding these terms to $\Delta^{Q P}$, one finds that the total correction of the optical gap to the bare potential $\left(T+V_{0}\right)$ gap is

$$
\Delta E_{g}^{\mathrm{opt}}=\int d \mathbf{r} \int d \mathbf{r}_{1} \frac{\rho_{c}(\mathbf{r})\left[\rho\left(\mathbf{r}_{1}\right)-\rho_{v}\left(\mathbf{r}_{1}\right)\right]}{\left|\mathbf{r}_{1}-\mathbf{r}\right|}
$$

(the Coulomb interaction of the electron with the valence charge density after excitation),

$$
-\int d \mathbf{r} \int d \mathbf{r}^{\prime} \sum_{v^{\prime} \neq v} \frac{\psi_{c}(\mathbf{r}) \psi_{v^{\prime}}(\mathbf{r}) \psi_{v^{\prime}}\left(\mathbf{r}^{\prime}\right) \psi_{c}(\mathbf{r})}{\left|\mathbf{r}-\mathbf{r}^{\prime}\right|}
$$

(the exchange interaction of the electron with the valence charge density after excitation),

$$
-\int d \mathbf{r} \int d \mathbf{r}_{1} \frac{\rho_{v}(\mathbf{r}) \rho\left(\mathbf{r}_{1}\right)}{\left|\mathbf{r}_{1}-\mathbf{r}\right|}
$$

(which subtracts the artificial Coulomb interaction of the now missing valence electron with valence charge density)

$$
+\int d \mathbf{r} \int d \mathbf{r}^{\prime} \sum_{v^{\prime}} \frac{\psi_{v}(\mathbf{r}) \psi_{v^{\prime}}(\mathbf{r}) \psi_{v}\left(\mathbf{r}^{\prime}\right) \psi_{v}(\mathbf{r})}{\left|\mathbf{r}-\mathbf{r}^{\prime}\right|}
$$

(which subtracts the artificial exchange interaction of the now missing valence electron with the valence charge density, and, for the nonoverlapping orbitals, exactly cancels the Coulomb self-interaction term).

This result is very intuitive. In order to get the TDDFT result, one could now simply take the form (6.13) of $V_{x}$ derived above for the case of nonoverlapping orbitals, and obtain a kernel

$$
f_{x}\left(\mathbf{r}, \mathbf{r}^{\prime}\right)=\frac{\delta V_{x}(\mathbf{r})}{\delta \rho\left(\mathbf{r}^{\prime}\right)}=-\frac{\delta_{R_{\mathbf{r}}, R_{\mathbf{r}^{\prime}}}}{\left|\mathbf{r}-\mathbf{r}^{\prime}\right|} .
$$

Hence in the diagonal approximation the optical gap would turn out to be identical to the Kohn-Sham eigenvalue gap, as in the case of only one electron, since $f_{x}$ cancels the Hartree part of the kernel. This is however not due to a failure of the exact-exchange approach; in fact, the kernel (6.15) has been derived from $V_{x}$ which was approximated for the case of the model of nonoverlapping valence states. Instead, one should first perform the functional derivative and then do the approximation. In that case, one gets additional terms, which should finally yield the correct result for the exchange-only case, as has been shown by Gonze and Scheffler (1999).

It is also interesting to note that in the case of nonoverlapping valence orbitals, the PGG kernel in the diagonal approximation yields the same (wrong) result as Eq. (6.15).

In summary, one should note that the exchangecorrelation kernel has to simulate the result of selfenergy corrections to the exact Kohn-Sham eigenvalues, plus the direct electron-hole interaction. In the oneoccupied-level limit cancellations occur (for example between the electron-hole interaction and the conduction- 
state matrix element of $V_{x c}$ ) which might be used as an input for the design of kernels in systems with more electrons. From the case of several well-localized occupied states, one can see the importance of a kernel that is derived from a $V_{x}$ that well reproduces not only the ground-state density, but also density variations.

\section{Relaxation and correlation}

It is also worthwhile to analyze and compare the effects of relaxation and correlation. For the $N \rightarrow N+1$ electrons excitation, one has of course a strong relaxation (Hartree-like for localized states, and through correlation for delocalized states). Then, when going over from an $N \rightarrow N+1$ to the $N \rightarrow N^{*}$ excitation, one has to subtract the spurious electron-hole interaction and exchange. Now there is also relaxation due to the presence of the hole. One can suppose that the relaxation due to the electron and the hole cancel to first order, so the total $N \rightarrow N^{*}$ problem should have much less relaxation than the $N \rightarrow N+1$ electron problem.

The scenario describing exchange and correlation effects in the one- and two-quasiparticle excitation spectra of many-body systems is hence the following: the screening that explicitly appears in the $G W$ self-energy comes from the electronic relaxation (classical or through correlation, as discussed in the introduction) due to the additional electron. The diagonalization of the $G W$ Hamiltonian takes into account the fact that the $G W$ potential is different from the initial potential, which can be either Hartree, Hartree-Fock, $V_{x c}$, or unknown if one does $G W$ from scratch. Then, the diagonalization of the Bethe-Salpeter equation creates a correlated electronhole wave function. Before the diagonalization, the electron-hole wave function is $\Phi\left(\mathbf{r}_{e} \mathbf{r}_{h}\right)=\psi_{v}^{*}\left(\mathbf{r}_{h}\right) \psi_{c}\left(\mathbf{r}_{e}\right)$, which is uncorrelated. After the diagonalization, $\Phi$ has the correlated form $\Phi_{\lambda}\left(\mathbf{r}_{e} \mathbf{r}_{h}\right)=\Sigma_{v c} A_{\lambda}^{v c} \psi_{v}^{*}\left(\mathbf{r}_{h}\right) \psi_{c}\left(\mathbf{r}_{e}\right)$. In a molecule or cluster one can assume that one would get some reasonable result even by taking into account only diagonal elements; this is an uncorrelated, but interacting, electron-hole pair. It might also happen that only one occupied state is contributing, and the resulting wave function $\Phi_{\lambda}\left(\mathbf{r}_{e} \mathbf{r}_{h}\right)=\psi_{v}^{*}\left(\mathbf{r}_{h}\right)\left[\Sigma_{c} A_{\lambda}^{c} \psi_{c}\left(\mathbf{r}_{e}\right)\right]$ would still be uncorrelated; only the electron has relaxed, i.e., adjusted itself to the fact that the hole is present. These mixing effects on the wave functions can in fact be easily visualized in a cluster (see, for example, Albrecht et al., 1998b). In a solid, on the other hand, taking just one valence Bloch state would mean that one does not include more than one $k$ point, i.e., one would not get an excitonic effect. As in the case of the one-particle excitation, Hartree relaxation alone is not enough to describe the solid: one must consider correlation.

On the other hand, in TDDFT (and in the timedependent Hartree approach) the off-diagonal elements immediately yield the correlated electron-hole pair, and the same discussion as above holds concerning the difference between an extended crystal and a finite system.

\section{Comparison of some applications}

In real applications, of course, details of the system already at the independent-particle level are often the main ingredient for a first explanation of spectra. For example, bandstructure effects turn out to be decisive in determining not only the existence of interband transitions as observed in optical spectroscopy, but also the properties of plasmonic excitations in simple and noble metals. Indeed, $a b$ initio calculations of the dynamical response have properly described the plasmon dispersion in a variety of metals (Quong and Eguiluz, 1993; Aryasetiawan and Karlsson, 1994; Maddocks et al., 1994a, 1994b; Fleszar et al., 1997; Ku and Eguiluz, 1999; Cazalilla et al., 2000). Since, however, the independentparticle approach often fails to yield quantitative, or even qualitative, agreement with experiment, it is useful to compare the performance of various approaches applied to these realistic cases.

In the previous section, we have shown or cited several examples for successful TDDFT calculations of the absorption spectrum of atoms and clusters. Far fewer examples exist for TDDFT calculations of the absorption spectrum of bulk materials. The influence of an adiabatic LDA kernel $f_{x c}$ on the absorption spectrum of silicon has been calculated by Gavrilenko and Bechstedt (1996), who showed that its effect is negligible. These results have been confirmed by Koostra et al. (2000, although no comparison to the RPA result is given in that paper), and can also be seen in Fig. 10. In fact, since the independent-particle response function $\chi_{0}$ goes to zero as $q^{2}$, any kernel that does not have a $1 / q^{2}$ divergence does not contribute to the head (i.e., the $\mathbf{G}=0, \mathbf{G}^{\prime}=0$ element) of the matrix $f_{x c} \chi_{0}$ and can at the best yield visible effects through off-diagonal elements, i.e., mediated by local field effects. Therefore it is often heard that "TDLDA works in clusters, but not in solids." We use this statement as a guideline for the following discussions and to provide some explanations. In order to allow for a meaningful comparison, it is useful to concentrate first on the effect of the part of the kernel which is common to the TDDFT and the Bethe-Salpeter approaches, namely, the $v$ (or $\bar{v}$ ) part. In this way one can single out the rest and determine to what extent TDDFT and the Bethe-Salpeter approaches, respectively, actually improve on $P$-the only quantity that is treated differently in the two approaches.

\section{Common ingredient: the bare Coulomb interaction}

As pointed out in Sec. II.B, the $\mathbf{G}=0$ element of $v$ determines the macroscopic screening $1-v_{0} P_{00}$, which accounts for the essential difference between absorption and EELS spectra of solids. The rest of the Coulomb interaction, i.e., $\bar{v}$, gives rise to what is called "local field effects" in the language of solid-state physics. This means that it reflects the fact that an inhomogeneous system will exhibit an electronic response that is position dependent (and not only distance dependent). It is intuitively clear that such an effect will be the stronger the more a system is inhomogeneous. It is also obvious that 
an atom, a molecule, or a cluster of whatever size is by itself a strong inhomogeneity in the empty space, and that the electronic response must depend strongly on the distance and on the position of the perturbation and the probe. ${ }^{46}$ Therefore one can expect that the effect of $\bar{v}$ alone will already be a strong modification of the spectra in a cluster, whereas in a solid it will depend on details of the charge density of the latter, with a tendency to have very weak effects in, say, nearly free electron metals (homogeneous systems) like simple metals. This is in fact the case. In Fig. 8 we have already shown the results for three different clusters, and discussed the fact that the Coulomb term has a very strong effect and removes most of the discrepancy between the LDA curves and experiment.

When moving to solids, a good example is the loss function of bulk silicon, in which the localization of the charge on the bonds gives rise to a non-negligible inhomogeneity. The results of local field effects on the plasmon resonance of silicon have already been discussed, for example, by Louie et al. (1975), who found that the local field effects are not as pronounced as in the case of the cluster, but are sizable. Moreover, as in the case of the cluster, agreement with experiment is improved when $\bar{v}$ is included; in particular, the height of the plasmon peak is considerably lowered.

When local field effects are included, good-quality loss spectra are also obtained in transition-metal compounds like $\mathrm{Ni}$ and $\mathrm{NiO}$ (Aryasetiawan, 1994). The good quality of RPA loss function results and the importance of local field effects is illustrated for rutile $\mathrm{TiO}_{2}$ in Fig. 11 (Vast et al., 2002). One can see that local field effects are particularly important at higher energies, in the region of transitions from the Ti $3 p$ semicore level, yielding good agreement with experiment. This almost seems to suggest that one could, in a first approach, always neglect the cumbersome exchange-correlation effects. This is of course not true. It is instructive to look, not only at the loss spectrum, but also at the absorption spectrum of bulk silicon $\left[\operatorname{Im}\left(\varepsilon_{M}\right)\right]$, shown in Fig. 5. The dot-dashed curve (RPA result) is in fact in poor agreement with experiment. Another striking example is the absorption spectrum of solid argon, shown in Fig. 12. The strong absorption peaks in the experiment (Saile, 1976; Saile et al., 1976) on the low-energy side, which are known to be of excitonic nature, are completely missing in both the RPA and the $G W$-RPA results (Olevano and Reining, 2001b). Note that local field effects are included in these calculations, but they cannot remove the discrepancies. The effect of $\bar{v}$ on $\operatorname{Im}\left(\varepsilon_{M}\right)$, in its more or less pronounced form, is in fact essentially to shift oscillator strength to higher energy. This is due to the fact that $\bar{v}$ is positive, being the Coulomb interaction be-

\footnotetext{
${ }^{46}$ The essential difference between even a very big cluster and an infinite solid is the presence of a surface between the cluster and vacuum, implying boundary conditions for the electric field.
}

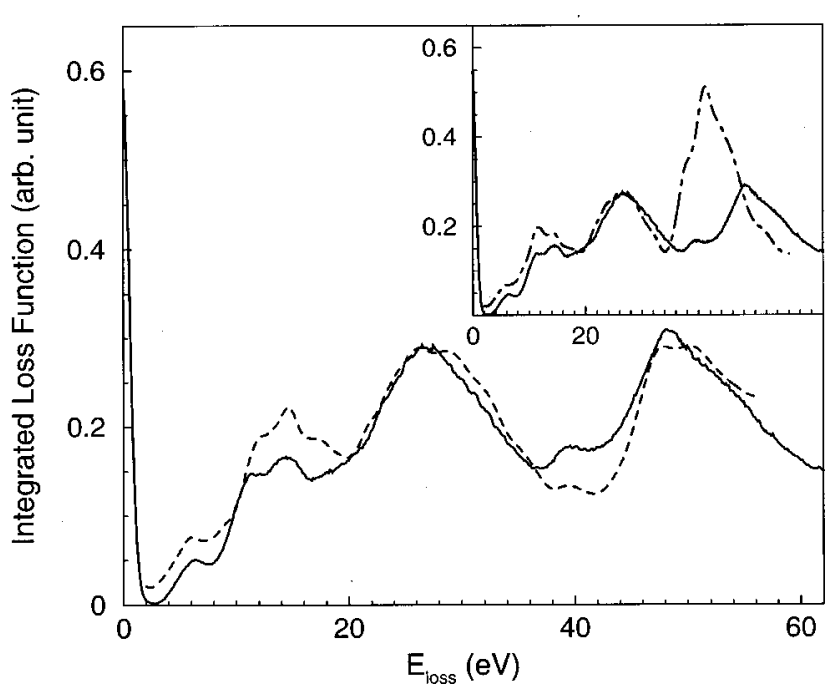

FIG. 11. Integrated loss function at $q \approx 0.4 \AA^{-1}$ for rutile $\mathrm{TiO}_{2}$ (Vast et al., 2002): solid line, experiment; dashed line, RPA results. The dot-dashed line in the inset shows the results without local field effects.

tween charge-density waves (Hanke and Sham, 1975). It never creates or even significantly enhances structures on the low-energy side.

Hence $\bar{v}$, or local field effects, are generally not sufficient to obtain quantitative (for $\varepsilon^{-1}$ ) or even qualitative (for $\varepsilon_{M}$ ) agreement between theory and absorption experiments, and one has to add the exchange-correlation effects. In other words, one has to use the interacting $P$ (instead of $P=P_{I Q P}$ ) in Eqs. (2.10) and (2.11). With respect to the RPA-plus-local-field result, these effects should cause a variety of modifications in the spectra, if they are supposed to restore agreement with experiment. Essentially, from the above results one can see

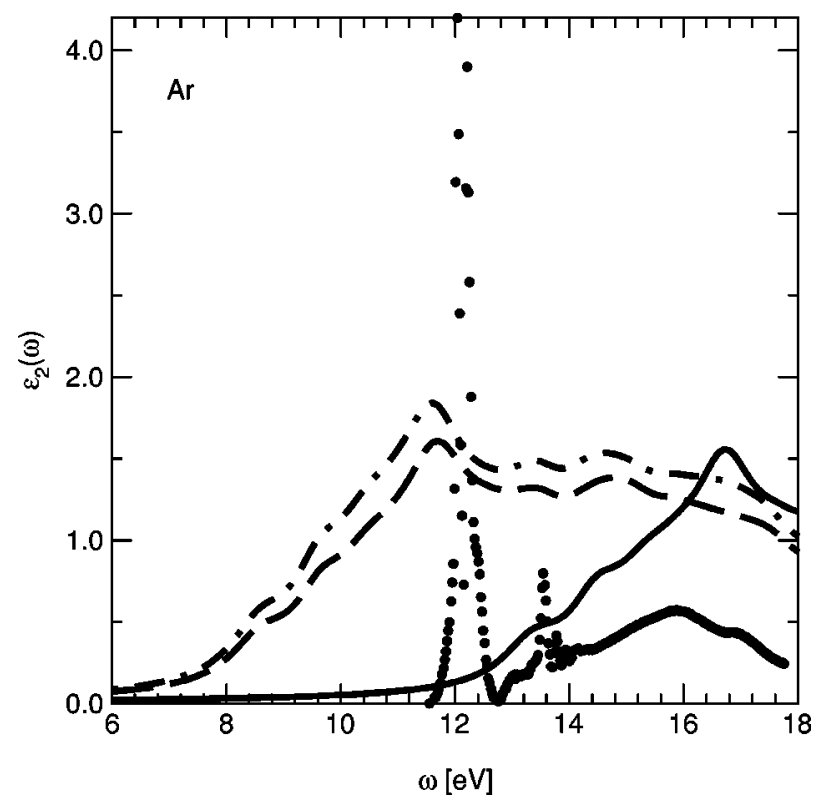

FIG. 12. Optical spectra for argon: - experiment (Saile, 1976); dashed line, RPA; dot-dashed line, TDLDA; solid line, $G W$-RPA. From Olevano and Reining, 2001b. 


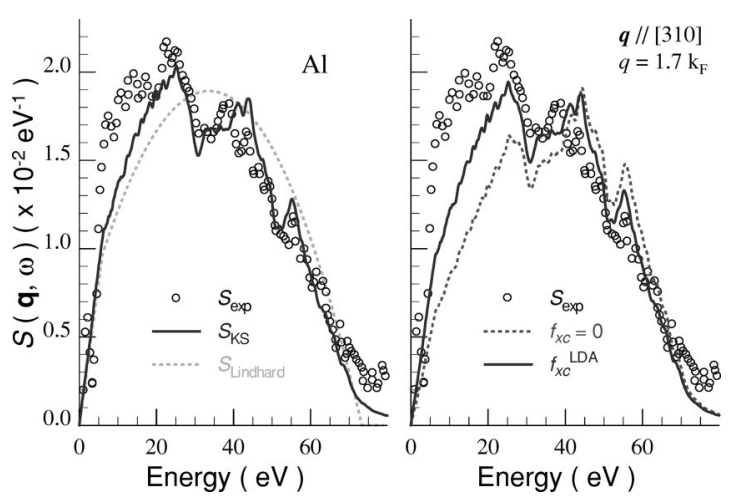

FIG. 13. Dynamical structure factor $S(\mathbf{q}, \omega)$ $=-2 \hbar \int d \mathbf{r} d \mathbf{r}^{\prime} e^{-i \mathbf{q}\left(\mathbf{r}-\mathbf{r}^{\prime}\right)} \operatorname{Im} \chi\left(\mathbf{r}, \mathbf{r}^{\prime}, \omega\right)$ of aluminum, calculations from Fleszar et al. (1995). Open circles are experimental data from Platzman et al. (1992). In the left panel, they are compared with the Kohn-Sham independent particle response (full line labeled $S_{K S}$, obtained taking $\chi=\chi_{0}$ in the above expression for $S$ ) (the double-bump structure was already obtained by Maddocks et al., 1994a, 1994b). The dotted line, labeled $S_{\text {Lindhard }}$, is the corresponding noninteracting response of jellium with $r_{s}=2.07$. In the right panel, the same experimental data are compared with $S$ calculated using $\chi$ from Eqs. (5.11) and (5.12). Full line, TDLDA $\left(f_{x c}\right)$ from Eq. (5.13); dashed line, $\operatorname{RPA}\left(f_{x c}=0\right)$.

that a shift is needed, which can be towards either lower energies (as in the case of the loss spectra of both clusters and solids) or higher energies (as in the case of the absorption spectrum of bulk silicon), together with a redistribution of oscillator strength towards lower energies, which may enhance existing peaks and/or create new ones, as in the case of solid argon (see Fig. 12).

\section{Exchange and correlation effects}

Concerning these exchange-correlation effects, the Bethe-Salpeter and the TDDFT approaches act in a completely different way. In fact, the Bethe-Salpeter approach can be seen as a two-step method, where first a $G W$ calculation yields a strong shift of the whole spectrum towards higher energies, and the subsequent inclusion of the electron-hole interaction redistributes oscillator strength towards the low-energy peaks, and eventually shifts peaks, or even creates peaks in the quasiparticle gap. It is clear that there must be a partial cancellation of the self-energy corrections and the electron-hole interaction. In the small cluster discussed above, and also for bulk plasmons, where the RPA plus local fields calculation already gives very good results, this cancellation is almost perfect. TDDFT, on the other hand, should describe directly the complete electronhole excitation, but in an effective way, which may be more difficult to understand [see also the discussion in Appendix A, after Eq. (A5)]. In this case, too, partial cancellations occur. Figure 13 shows an example of these cancellations. Fleszar et al. (1995) have calculated the dynamical structure factor of bulk aluminum within TDDFT. The independent-particle response $\chi_{0}$ closely simulates the measured spectrum, and even the double- hump structure is correctly predicted. However, when a full RPA calculation, including the Coulomb kernel, is performed, the result worsens. Exchange-correlation effects should hence completely cancel the Coulomb repulsion in that case (i.e., a kernel $f_{x c}=-1 /\left|\mathbf{r}-\mathbf{r}^{\prime}\right|$ would do the job). Depending on the situation and the system, different aspects of $f_{x c}$ will become important. For example, Sturm and Gusarov (2000) have shown that in order to describe the aluminum inelastic x-ray scattering cross section for large momentum transfer and large $\omega$, dynamical correlation effects in $f_{x c}$ are more important than band-structure effects (interband transitions are insufficient to explain the observed magnitude of the highenergy tail of the spectrum, while multiple particle excitations give the correct order of magnitude).

\section{a. Some applications to finite systems}

For clusters, the majority of DFT-based calculations today rely on the use of the static LDA kernel, since the optical spectra turn out to be in very good agreement with experiment even at the TDLDA level (Casida, 1995; Rubio et al., 1996; Yabana and Bertsch, 1996; Vasiliev et al., 1999, 2002). Although, as pointed out above, the improvement with respect to a static LDA calculation essentially comes from the Coulomb part of the kernel, and not from $f_{x c}$, these findings have led to an important breakthrough in the calculation of excitation spectra of finite systems and make the TDDFT technique very popular. One example is the benzene molecule (Fig. 14). It is clear that apart from small differences due to the resolution of vibrational modes in the experimental spectrum, the overall response is very well described by the TDLDA approach. ${ }^{47}$ Satisfactory agreement is also obtained for other molecular clusters as shown in Fig. 8. This figure however, also shows the need for improvements over the simple TDLDA.

A good and widely studied example is the silane molecule $\mathrm{SiH}_{4}$, for which quantum Monte Carlo (Grossman et al., 2001), Bethe-Salpeter (Rohlfing and Louie, 1998a, 2000; Grossman et al., 2001), and TDDFT results (Ögüut et al., 1997; Marques et al., 2001) are available. In particular, TDLDA or gradient corrected functionals predict that bound excitations will be resonances. This artifact is clearly corrected by the exact-exchange potential and a method proposed by van Leeuwen and Baerends (1994) to impose the correct asymptotic behavior on the exchange potential (Marques et al., 2001). Within this scheme the TDDFT results for $\mathrm{SiH}_{4}$ have the same ac-

\footnotetext{
${ }^{47}$ For the chiral $\mathrm{C}_{76}$ fullerene, TDLDA also provides a consistent description of the optical absorption spectrum, the circular dichroism spectrum, and the optical rotatory power (except for an overall shift of the total spectrum; Yabana and Bertsch, 1999a, 1999b). Similarly, the optical spectrum can be used to elucidate the ground-state structure of the smallest possible carbon cage $\mathrm{C}_{20}$ (Castro et al., 2001), which has been elusive to ground-state calculations in both quantum Monte Carlo (Grossmann et al., 1995) and DFT (Jones and Seifert, 1997).
} 


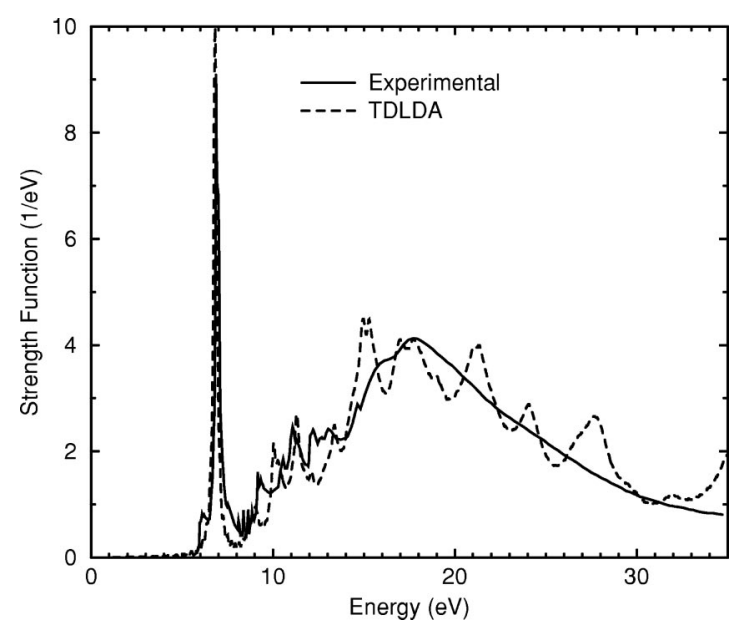

FIG. 14. Optical absorption of the benzene molecule, in units of $\mathrm{eV}^{-1}$. The TDLDA calculation is from Yabana and Bertsch (1999a), whereas the experiment is from Koch and Otto (1972).

curacy as the $G W$ or quantum Monte Carlo calculations. ${ }^{48}$ This fact is illustrated in Fig. 15.

TDLDA gives good results for $\mathrm{Na}_{4}$ as well as for small and large clusters of simple and noble metal atoms $(\mathrm{Ru}-$ bio and Serra, 1993; Rubio et al., 1996, 1997; Yabana and Bertsch, 1996; Serra and Rubio, 1997; Vasiliev et al., 1999, 2002) and fullerenes (Yabana and Berstch, 1999a, 1999b; Castro et al., 2001) but works less well in small hydrogenated silicon clusters (see Fig. 15). ${ }^{49}$ This problem might be due to the presence of more localized bonds. The exact-exchange calculation does help in this case and keeps nearly the same accuracy as in the sodium cluster. Both calculations are in slightly better agreement with experiment than the Bethe-Salpeter calculation (Onida et al., 1995). Note that the two peaks at about $3 \mathrm{eV}$ in both the exact-exchange and the BetheSalpeter calculations are very similar. However, in both clusters we see an overall tendency of the TDDFT calculations with approximate kernels to give larger excitation energies (peak positions blueshifted with respect to experiment). By looking carefully at the different contributions coming from the correct asymptotic behavior of the potential, as well as at variations of the $f_{x c}$ kernel, Marques et al. (2001) show that there is an inherent

\footnotetext{
${ }^{48}$ Very good results are obtained in the three approachesquantum Monte Carlo, Bethe-Salpeter and TDDFT with exact exchange. In particular, they overestimate the energy of the first singlet excitation by $3 \%, 4.5 \%$, and $1.5 \%$, respectively, whereas the ionization potential (exactly reproduced in quantum Monte Carlo) is only $4 \%$ overestimated by TDDFT with exact exchange. The quantum Monte Carlo results are equivalent to the best quantum-chemical (coupled-cluster and complete active space self-consistent-field) calculations (for details see Grossmann et al., 2001 and references therein).

${ }^{49}$ Here it is important to remark that for the optical gaps, the calculated values within TDLDA for large-size hydrogenated silicon clusters (Öğüt et al., 1997; Vasiliev et al., 2001) agree to within $\simeq 10 \%$ with experiment.
}
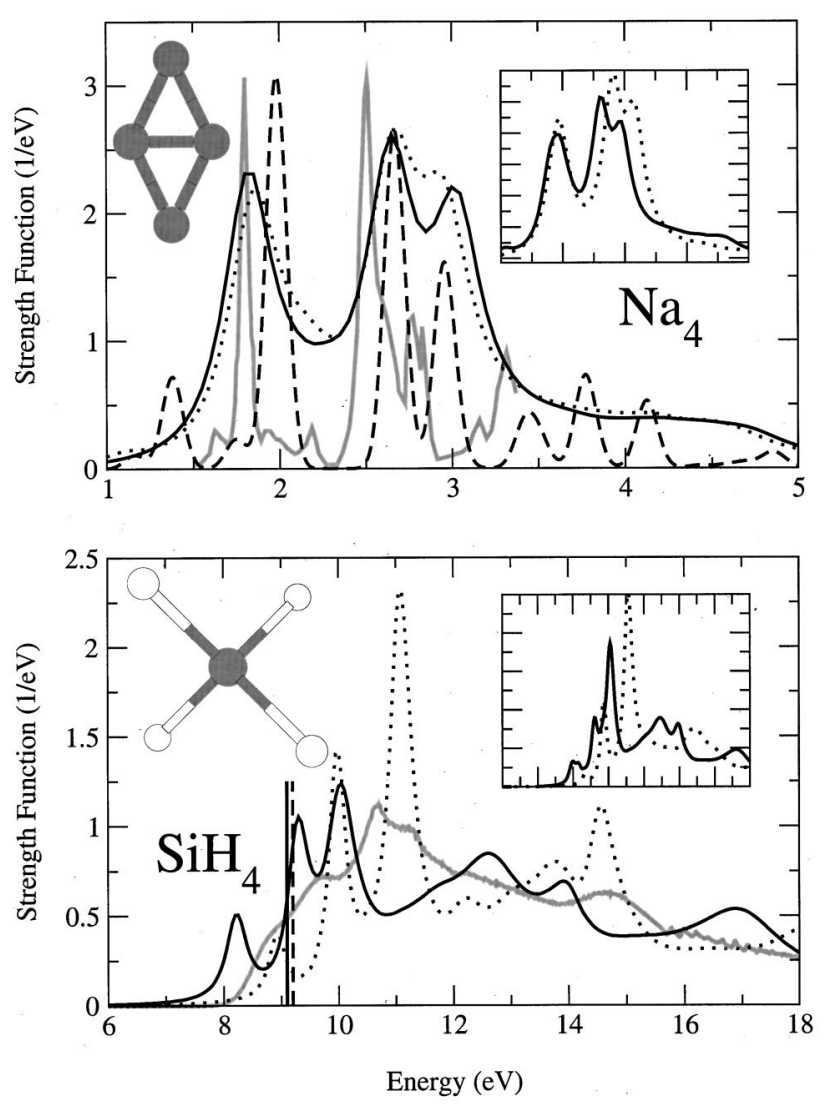

FIG. 15. Calculation of the strength function for $\mathrm{Na}_{4}$ and $\mathrm{SiH}_{4}$ within TDDFT using different kernels (Marques et al., 2001): solid line, TDLDA; dotted line, exact-exchange. Gray solid line, experimental results; dashed line, the Bethe-Salpeter equation results of Onida et al. (1995) for $\mathrm{Na}_{4}$. Vertical lines are the lowest excitation energies obtained by Grossman et al. (2001) for $\mathrm{SiH}_{4}$ : solid vertical line, quantum Monte Carlo calculations; dashed vertical line, Bethe-Salpeter equation. In the inset: solid line, self-interaction correction; dotted line, van Leeuwen and Baerends (LB94) prescription.

source of error in the approximated kernels coming from neglect of dynamical (correlation) effects. These tend to shift the spectrum to lower energies and are also responsible for improving the excitation energies of Rydberg-like states (where exchange effects are small due to the nearly zero overlap between the Rydberg unoccupied state and the other valence states; thus the usually weak polarization effects are going to play an important role here).

For these small molecules, there are thus various methods to get good transition energies: quantum Monte Carlo, the Bethe-Salpeter equation, and TDDFT yield satisfactory results. One might of course wonder whether the calculation of spectra involving empty (extended) states is not questionable in a supercell approach. In fact, high-energy, extended states do represent a problem if they are really needed as the physical final (one-electron) state (e.g., in absorption at higher frequency, or for a photoemitted electron, if one wants to go beyond the usual approximation of neglecting the final-state effects). Most often, however, these states are used only as intermediate states in summations, i.e., for 
a closure relation, which is a mathematical property deriving from the completeness of the basis set. Since the validity of the closure relation does not depend on the chosen boundary conditions (even if the actual shape of the individual states does), this does not present a problem.

Concerning the Bethe-Salpeter approach for $\mathrm{SiH}_{4}$, it should be noted that (i) the results depend on the choices made for the different steps of this rather complicated technique, e.g., the diagonalization of the $G W$ Hamiltonian discussed above; and (ii) only transition energies, not line shapes, are shown. However, in TDDFT the results depend of course on the choice made for the kernel, as discussed above.

\section{b. Some applications to extended systems}

In solids one should look at both absorption and electron-energy-loss spectra. As discussed in the previous sections, TDLDA fails to describe the absorption spectra of solids. In fact, at least in the case of silicon, the failure of the TDLDA $f_{x c}$ to reproduce the correct long-range behavior of the kernel explains its bad performance, as shown above in Fig. 10. On the other hand, Olevano and Reining (2001a) have calculated the electron-energy-loss spectrum of silicon including local field effects, $G W$ corrections, and excitonic effects, for vanishing momentum transfer. The inclusion of excitonic effects improves the results with respect to both the RPA and, more drastically, the $G W$ calculations (see Fig. 16): $G W$ and excitonic corrections cancel to a large extent. In contrast to the case of small molecules and to absorption spectra in bulk, here the correct result could only be obtained by taking into account the coupling between transitions at positive and negative frequencies [blocks $K$ in Eq. (B20)]. Figure 16 also shows that TDLDA improves with respect to the RPA calculation (including local field effects), which means that even the TDLDA $f_{x c}$ has a visible effect on the electron-energyloss spectrum. However, as in the case of clusters, local field effects are more important than $f_{x c}$ for the electron-energy loss spectra. This is a very general finding in TDLDA electron-energy-loss calculations (see the results of Waidmann et al., 2000 and references therein).

For nonvanishing momentum transfer, one can compare the TDDFT results of Waidmann et al. (2000) on diamond to the Bethe-Salpeter results of Caliebe et al. (2000). The comparison is limited, however, to the lowenergy region well below the plasmon. First of all, the work of Waidmann et al. (2000) shows the increasing importance of local field effects with increasing $q$. Local field effects start to be visible at $q=1.0 \AA^{-1}$ (about 1.1 $\Gamma X$ ). At $q=1.5 \AA^{-1}$ (about $1.7 \Gamma X$ ) they are already very strong. Second, TDLDA gives a reasonable description of the low-energy part of the spectrum. It should be pointed out that the authors state that the LDA kernel $f_{x c}$ itself has only a negligible effect on this result. On the other hand, in the work of Caliebe et al. (2000) the Bethe-Salpeter method is applied to the calculation of the same spectra. Results of comparable quality are obtained in the end. A more detailed com-

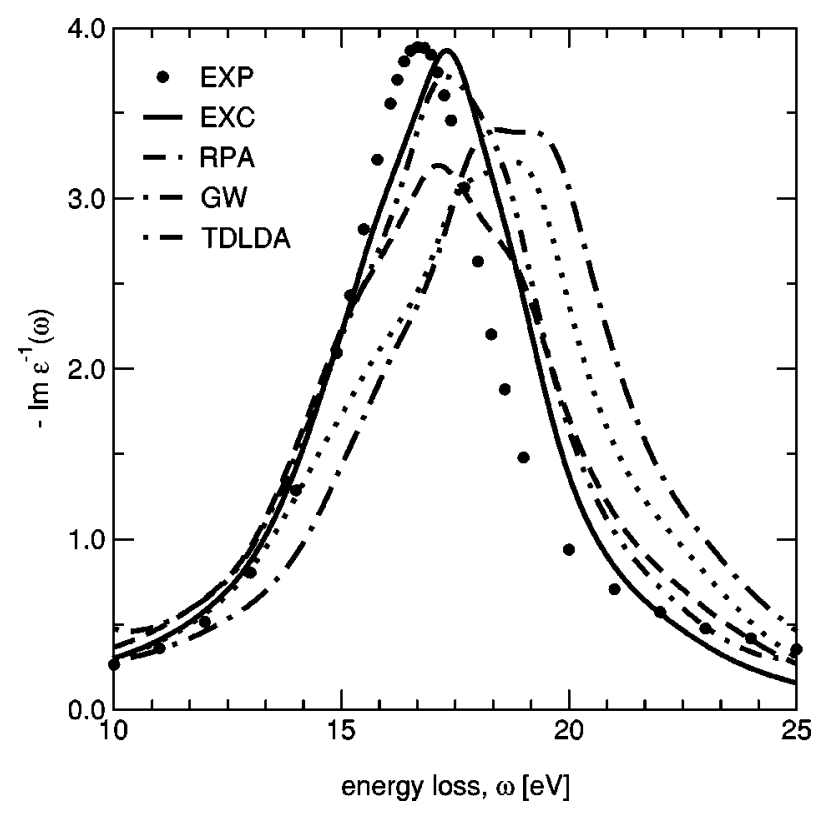

FIG. 16. Energy-loss spectra of bulk silicon: $\bullet$, experiment (Stiebling, 1978); dotted line, Bethe-Salpeter equation without resonant-antiresonant coupling; dash-dotted line, $G W$-RPA; dashed line, RPA; dash-double-dotted line, TDLDA; solid line, Bethe-Salpeter equation with coupling (Olevano and Reining, 2001a).

parison is difficult, since the Bethe-Salpeter results are compared to those obtained when $G W$ corrections are added to the Kohn-Sham eigenvalues in the independent-particle polarization. Moreover, as is often the case in the Bethe-Salpeter approach, the local field effects are treated as part of the electron-hole interaction kernel. In other words, part of the electron-hole effect shown is due to the local field effects. The net improvement due to the electron-hole interaction is hence very strong (although not systematic for all spectra), but it is difficult to estimate the total improvement due to self-energy plus electron-hole attraction effects, in comparison to RPA (including local field effects), or better, TDLDA, results. A detailed comparison of TDDFT and Bethe-Salpeter results for the $q \neq 0$ case is in fact still missing in the literature. Bethe-Salpeter results should be superior to TDLDA results in those parts of the loss spectra that are dominated by interband transitions, i.e., by the structures in the imaginary part of the dielectric function, which are not well reproduced in TDLDA.

\section{CONCLUSIONS-FREQUENTLY ASKED QUESTIONS AND OPEN QUESTIONS}

Since the very beginning of physics and chemistry, the interaction of photons and electrons with matter has been a major topic of study. Today, most characterization tools as well as electro-optical devices are based on our understanding of these interactions. Technological applications are rapidly progressing, but many fundamental questions concerning theoretical and numerical descriptions are still open. 
This has motivated us to discuss in the present review the two most widely used techniques for describing electronic excitations in finite and infinite systems, namely, the Green's-function approach to many-body perturbation theory calculations, and time-dependent densityfunctional theory. We have presented the foundations and approximations of the two approaches as well as the advantages and drawbacks of the methods. A special effort has been made to provide a unified picture of the underlying equations, in order to facilitate the comparison and to analyze the most frequently used approximations. We have stressed the fact that the two approaches are somewhat complementary and that one should work on both sides in order to optimize the search for systematic improvements.

We have not presumed to give a complete view of all theoretical descriptions of spectroscopies, but rather looked at the field from a particular point of view, with the aim of motivating some possible future developments. Some points have been treated in more detail than others; we have made our choices on the basis of the very lively discussions which are now going on in this field. As a conclusion, we summarize below a set of frequently asked questions, which we have tried to answer in the present paper. Many points are not new, and they will be obvious for a specialized reader, but we think that it is useful to put them in a common context. This should also be helpful to those who wish to enter the field.

Other questions do not have an answer yet. They will be treated at the end of this section-as a motivation for future research.

- The delta-self-consistent field approach, i.e., explicitly calculating total energy differences, is known to work well. Why can't we just always calculate total energy differences in DFT, instead of struggling with the meaning of Kohn-Sham eigenvalues? First, $\triangle \mathrm{SCF}$ in DFT supposes that one can simulate the initial and the final state of the excitation by occupying selected oneparticle orbitals. This excludes excitations that are not easily described in terms of isolated single-particle transitions (for example, the collective plasmon excitations). Second, whereas the choice of the $N$ and $N$ \pm 1 states can be straightforward in a small system, it is not necessarily well defined in the bulk; in fact, when Bloch states are chosen to describe the electrons, Hartree relaxation effects vanish and hence the main advantage of $\triangle \mathrm{SCF}$ is lost. One should then include dynamical correlation effects, which go beyond static DFT- $\Delta$ SCF. See discussions in Secs. IV.A.2 and VI.C.

- Does TDDFT give electron removal or addition energies? In Sec. V we described how TDDFT provides an exact framework for getting the excitation energies of an $N$-particle system. The theory handles only neutral excitations, that is, excitations in which the number of particles does not change. Concerning $N \rightarrow N \pm 1$ processes, TDDFT is only supposed to have the correct ionization potential threshold, as this should be guar- anteed by the exact DFT exchange-correlation potential (Almbladh and von Barth, 1985).

- If quasiparticle energies are electron addition and removal energies, i.e., total energy differences, how can they be complex? In the Lehmann representation of the Green's function the excitation energies involved are always differences of total energies between the $N$ and $N \pm 1$ systems; hence they are always real. The infinitely close-lying peaks of the spectral function in the Lehmann representation merge into broad structures that can be identified as quasiparticle peaks. These structures have a finite width and can hence be described by a real part (peak position) and an imaginary part (width) of a quasiparticle's energy. See discussion in Sec. III.A.

- Why can TDDFT yield absorption spectra, when DFT is a ground-state theory? DFT is a ground-state theory because it is based on a minimization of the total energy. TDDFT, on the other hand, is derived from the extrema of the quantum-mechanical action, and hence describes the evolution of the system instead of its ground state. This difference is equivalent to that in classical mechanics, where the equilibrium position of a particle in space can be found by looking for the minimum of the potential, whereas its trajectory is derived from the extrema of the classical action. See discussion in Sec. V.

- Can one in principle get excitonic effects in TDDFT? Yes, excitonic effects are in principle exactly contained in the TDDFT equations, if the exact exchangecorrelation potential $V_{x c}$ and kernel $\left[f_{x c}\left(\mathbf{r}, \mathbf{r}^{\prime}, \omega\right)\right]$ are used. However, the practical implementations use simple functionals, which lack, for example, the proper spatial nonlocality. Therefore direct electronhole interaction effects are only partially described in finite systems, and in general still out of reach of today's TDDFT calculations for solids. See Sec. V.C and Appendix C.

- Is the fact that Kohn-Sham eigenvalue differences underestimate the gap a problem for the calculation of absorption spectra within TDDFT? No. The KohnSham eigenvalue differences are not meant to reproduce the (optical) gap. The optical gap is determined by the Kohn-Sham potential (eigenvalues) and by variations of the Kohn-Sham potential, through the kernel. This latter contribution changes the optical gap with respect to the Kohn-Sham eigenvalue difference. Of course, results improve when better potentials (eigenvalues) are used-but "better" does not mean "close to quasiparticle energies."

- Why does the RPA often yield good results for electron-energy loss, but not for absorption in solids? This can be traced back to the crucial role played by the long-range part of the Coulomb potential in the kernel: the exchange-correlation contribution $f_{x c}$ is in fact added to the Hartree term $v$ in the case of the loss spectra [see Eqs. (2.3) and (2.11)], but to the difference between the bare $v$ and the long-range part 
$v(\mathbf{G}=0)$ in the case of absorption [see Eqs. (2.9) and (2.10)]. In the latter case, the only long-range term comes from $f_{x c}$, which makes it so crucial. See Secs. II.B and VI.D.

- Why does TDLDA work for clusters, but not for the absorption spectra of solids? First, the main difference between the results of TDLDA and those of Eq. (2.8) [the IP-RPA dielectric function] in clusters stems from the contribution $v$ in the kernel, which is always exact. Second, for finite systems $f_{x c}^{T D L D A}$ provides an effective electron-hole attraction, which gives a significant contribution to the spectra. In infinite nonmetallic systems, the LDA $f_{x c}$ only contributes through local field effects, since the head of the matrix $f_{x c} \chi_{0}$ vanishes as $q \rightarrow 0$. See Secs. V.C, VI.D, and Appendix C.

- What is the link between the Hartree potential, the electron-hole exchange interaction, and local field effects in a solid? The variation of the Hartree potential with respect to the density yields a contribution $v$ to the kernel of both the TDDFT and the BetheSalpeter equations. This contribution has to be taken with or without $(v$ or $\bar{v})$ its long-range $(\mathbf{G}=0)$ contribution in the case of electron-energy loss or absorption, respectively. Neglecting $\bar{v}$ in the screening equations is equivalent to neglecting local field effects. The matrix elements of $v$ and $\bar{v}$ in an electron-hole basis are dipole-dipole like, and one calls this contribution therefore the "electron-hole exchange" in the BetheSalpeter framework. See Secs. II.B and IV.B.2 for more details.

- Is the electron-hole exchange interaction screened or unscreened? The electron-hole exchange interaction stems from the density variation of the Hartree potential and is therefore exactly the unscreened Coulomb interaction (see Secs. II.B, II.C, and IV.B.2). Sometimes model calculations can be found in the literature where this interaction is screened. This procedure is justified when (and only when) an extremely restricted space of transitions is explicitly mixed by the exciton; the neglected transitions are then implicitly put back into the calculation by an effective screening of the electron-hole exchange.

- To describe two-particle excited states one resorts to the two-particle Bethe-Salpeter equation. What about three-, four-, or more-particle excitations? Do we need three- or more-particle analogies to the Bethe-Salpeter equation? No. In fact, even for the case of two-particle excited states we do not need the two-particle BetheSalpeter equation, since it would be formally sufficient to solve Hedin's equation for the vertex [Eq. (3.25)]. The situation is similar to the case of electron addition or removal energies, where the set of higher-order entangled Green's functions is decoupled by introducing the concept of the self-energy, which implicitly contains all higher-order electron interactions. Similarly, the complex equation for the polarization operator in terms of higher-order polarization operators can be formally solved by introducing the vertex function. See Sec. IV.B.

- TDDFT equations can be written as a quadratic equation (6.3) or as a Dyson-like equation (5.11): are the two forms equivalent, and what are the reasons to choose one of them? Yes, the two forms are indeed equivalent. The TDDFT equation can be written for four-point response functions or for two-point (contracted) functions [see Eqs. (2.15) and (2.19)]. The two-point formulation (which does not exist for the Bethe-Salpeter equation) leads to the Dyson-like equations for two-index matrices [Eq. (5.11)], with the obvious advantage of a better scaling with the system size. On the other hand, the four-point formulation allows one to switch easily to transition space and to compare directly with the Bethe-Salpeter equation. The transition-space formulation can be written as an eigenvalue equation like Eq. (5.15) or as a quadratic equation for $\omega$ like Eq. (6.3). It is advantageous for cases in which only a limited number of transitions contribute to the spectrum. See Secs. V.B, VI.A, and II.C.

- Since most calculations use supercells or finite domains, can we trust the higher-energy eigenstates, which can "sense" the boundary conditions? If one is explicitly interested in these states, that can actually be a big problem. However, most often (e.g., in the calculation of a Green's function) they are just virtual states that are summed over, and one does not care. See also Sec. VI.D.2.a.

One important question now is actually which way one should go-is the Bethe-Salpeter equation or TDDFT more promising? Since both approaches are exact, the answer depends of course on whether-and when-the remaining open questions in the two approaches can be solved. Some questions are common to both. An important point is whether pseudopotentials are always adequate for describing spectra with the precision one asks for today. For certain applications, one should perhaps migrate towards all-electron schemes. Other questions are specific to the Bethe-Salpeter or the TDDFT approach.

The Bethe-Salpeter approach offers a clear physical picture and straightforward possibilities for the analysis of results. It seems to work over a wide range of systems. The TDDFT approach, on the other hand, is appealing since it calculates things in a more direct way (without passing through electron addition and removal energies) and is, in principle, easier to use.

Open problems in the Bethe-Salpeter approach include, of course, the need for technical developments to overcome the bottlenecks linked to the four-point equations. Moreover, just as in $G W$, not all the "ingredients" of the method are uniquely defined: how, for example, should vertex corrections and dynamical screening be included consistently? It is clear that the level of approximation which should be used for each ingredient in 
the various Green's-function-based approaches is a delicate and nontrivial question. One should wonder why in the calculation of an electron-energy-loss spectrum it is a good approximation to use $\varepsilon^{-1}$ constructed from a $P$ $=P_{I Q P}$ with DFT eigenvalues, and a much worse one when $G W$ eigenvalues are used, whereas in $G W$ calculations, especially for large-gap systems, an update of the energies entering in $\varepsilon^{-1}$ yields improvements for the resulting quasiparticle energies. At first sight, this seems contradictory. At this point we propose that one answer may be to put all choices on the basis of a consistent iteration scheme. This means that one starts with a $G W$ calculation and subsequently iterates Hedin's equations. The iteration first yields $P$, which contains a vertex correction. This explains why the electron-energy-loss spectrum using $P=P_{I Q P}$ with $G W$ eigenvalues yields poor results, but using $P$ with $G W$ eigenvalues and a vertex correction yields good results. Then one can go further and put this $P$ into the equation for $\Sigma$. Now a second vertex is appearing, and it is has been shown that there are cancellations between the vertex in $P$ and the explicit one in $\Sigma$. It is known that it is better to consistently neglect the vertex in both $W$ and $\Sigma$, which in practice means that a simple update of the energy denominator is in general the most consistent thing to do in a $G W$ calculation, if one does not want to include both vertices. Note that one quickly runs into trouble when also updating the wave functions, because of the dynamical effects, whereas it sometimes turns out to be necessary to update their spatial behavior. These are handwaving arguments, though essentially these-and the success of an approach in real calculations-justify the choices that are made in the iteration of the equations. More successes-and failures-of the Bethe-Salpeter approach in its actual form are needed in order to sort out this question.

In TDDFT, calculations are less cumbersome (if the two-point representation is chosen) than in the BetheSalpeter method at the moment, i.e., using the simple kernels available today, like that of the adiabatic LDA. Better exchange-correlation potentials and better kernels have to be found, especially if TDDFT is meant to become a method for the calculation of absorption spectra in solids. These kernels might well turn out to be very complicated, and as difficult to treat as the BetheSalpeter equation. There is some hope that, at least in certain cases, relatively simple solutions can be found (see Appendix C). The Bethe-Salpeter equation seems to provide a good starting point for the derivation of such effective kernels, since it contains the essential physics in a structure that is actually close to that of TDDFT.

In conclusion, it seems reasonable to suppose that progress will come from a common effort of people working in the fields of the Bethe-Salpeter equation and of TDDFT.

\section{ACKNOWLEDGMENTS}

We thank C. O. Almbladh, F. Aryasetiawan, P. Ballone, F. Bechstedt, M. Cini, R. Del Sole, J. Dobson, R.
Godby, L. Hedin, C. Hogan, I. Nagy, V. Olevano, F. Sirotti, F. Sottile, N. Vast, and U. von Barth for helpful discussions, collaborations, and critical reading of the manuscript. We are grateful for collaboration and discussions with G. Bertsch, A. Castro, G. Colò, P. M. Echenique, W. Ekardt, E. K. U. Gross, O. Gunnarsson, S. G. Louie, M. Marques, A. Schindlmayer, E. Shirley, and K. Yabana. We thank all the participants of the $2000 \mathrm{CE}$ CAM workshop on Electronic Excitations, where the idea of writing the present review was born. We have also benefited from insights and discussions provided by the series of workshops on this subject organized at the Ecole Polytechnique, Rome, Valladolid and CECAM since 1996. We thank A. Castro and V. Olevano for making some of the figures used in this review. This work was supported by the RTN program of the European Union NANOPHASE (contract HPRN-CT-200000167). G.O. acknowledges INFM for financial support (project PRA 1MESS). Computer time has been granted by IDRIS (CNRS, France) on project 010544.

\section{APPENDIX A: EXACT PROPERTIES OF $f_{x c}$}

Although the development of time-dependent functionals is still at a very early stage compared to that of static functionals, some of the known results for a homogeneous gas can be generalized to the inhomogeneous case. For example, causality leads to Kramers-Kronig relations for the real and imaginary parts of $f_{x c}\left(\mathbf{r}, \mathbf{r}^{\prime}, \omega\right)$, and the fact that $f_{x c}\left(\mathbf{r}, t ; \mathbf{r}^{\prime}, t^{\prime}\right)$ is a real-valued quantity implies that $f_{x c}\left(\mathbf{r} ; \mathbf{r}^{\prime}, \omega\right)=f_{x c}\left(\mathbf{r} ; \mathbf{r}^{\prime},-\omega\right)^{*}$. Besides that, the response functions satisfy the spatial symmetry relations $\chi\left(\mathbf{r}, \mathbf{r}^{\prime}, \omega\right)=\chi\left(\mathbf{r}^{\prime}, \mathbf{r}, \omega\right)$, provided that the unperturbed system has time-reversal symmetry (no magnetic field is applied or generated in the system). Thus one finds that $f_{x c}\left(\mathbf{r}, \mathbf{r}^{\prime}, \omega\right)=f_{x c}\left(\mathbf{r}^{\prime}, \mathbf{r}, \omega\right)$.

Further constraints on the potential $V_{x c}$ and kernel $f_{x c}$ can be deduced from the quantum-mechanical equation of motion applied to the position operator $\hat{\mathbf{r}}$ : $(d / d t\langle\Psi(t)|\mathbf{r}| \Psi(t)\rangle=i\langle\Psi(t)|[H(t), \hat{\mathbf{r}}]| \Psi(t)\rangle)$. The final and rigorous result is (Gross et al., 1996)

$$
\int d \mathbf{r} \rho(\mathbf{r}, t) \nabla V_{x c}[\rho](\mathbf{r}, t)=0 .
$$

This relation is also satisfied by the Hartree potential. This is nothing other than a mathematical statement of the physical fact that the exchange-correlation potential does not exert a net force on the system. One also obtains the corresponding "zero-torque" expression by considering the angle operator $\hat{\varphi}$ and using the rotational invariance of the Coulomb interaction, yielding

$$
\int d \mathbf{r} \rho(\mathbf{r}, t) \mathbf{r} \times \nabla V_{x c}[\rho](\mathbf{r}, t)=0 .
$$

Corresponding properties of the exact exchangecorrelation kernel are obtained by evaluating the two previous expressions at the density $\rho(\mathbf{r}, t)=\rho_{0}(\mathbf{r})$ $+\delta \rho(\mathbf{r}, t)$ for arbitrary $\delta \rho(\mathbf{r}, t)$. In frequency space one gets 


$$
\begin{aligned}
& \int d \mathbf{r} \rho_{0}(\mathbf{r}) \nabla_{\mathbf{r}} f_{x c}\left[\rho_{0}\right]\left(\mathbf{r}, \mathbf{r}^{\prime}, \omega\right)=-\nabla_{\mathbf{r}^{\prime}} V_{x c}\left[\rho_{0}\right]\left(\mathbf{r}^{\prime}\right), \\
& \int d \mathbf{r} \rho_{0}(\mathbf{r}) \mathbf{r} \times \nabla_{\mathbf{r}} f_{x c}\left[\rho_{0}\right]\left(\mathbf{r}, \mathbf{r}^{\prime}, \omega\right)=-\mathbf{r}^{\prime} \times \nabla_{\mathbf{r}^{\prime}} V_{x c}\left[\rho_{0}\right]\left(\mathbf{r}^{\prime}\right) .
\end{aligned}
$$

Concerning the electronic damping mechanism which is included in $f_{x c}$, we should emphasize that the standard static approximations for $f_{x c}$ [where $f_{x c}(\omega):=f_{x c}(\omega=0)$ is a real quantity] based on the homogeneous gas predict an infinite lifetime for plasmon excitations at small wave vectors. This artifact is inconsistent with the fact that the exact kernel has an imaginary part related to the multipair component of the response function $\chi_{m p}(q, \omega)$ (Sturm and Gusarov, 2000), that is,

$$
\operatorname{Im}\left[f_{x c}(\mathbf{q}, \omega)\right] \simeq-\left[\frac{\omega}{\omega_{p}}\right]^{4} v(\mathbf{q})^{2} \operatorname{Im} \chi_{m p}(\mathbf{q}, \omega),
$$

where $w_{p}$ is the plasmon frequency. These higher-order interactions are beyond the usual description of screening within the RPA or bubble approximation, and involve the partial summations of many complex diagrams appearing in the many-body response function.

In the homogeneous electron gas some exact relations for the $f_{x c}$ kernel must hold. In particular one has the following relations:

the compressibility sum rule, $\lim _{q \rightarrow 0} f_{x c}(q, \omega=0)$ $=d^{2} \rho \epsilon_{x c}(\rho) / d \rho^{2}$, where $\epsilon_{x c}(\rho)$ denotes the exchange-correlation energy per particle;

the third frequency moment sum rule:

$$
\begin{aligned}
\lim _{q \rightarrow 0} f_{x c}(q, \omega=\infty)= & -\frac{4}{5} \rho^{2 / 3} \frac{d \epsilon_{x c}(\rho) / \rho^{2 / 3}}{d \rho} \\
& +6 \rho^{1 / 3} \frac{d \epsilon_{x c}(\rho) / \rho^{1 / 3}}{d \rho} ;
\end{aligned}
$$

(iii) the static and frequency-dependent shortwavelength behavior: $\lim _{q \rightarrow \infty} f_{x c}(q, \omega=0) \propto c$ $-\left(b / q^{2}\right)[1-g(0)]$ [the correct values of the constants $b$ and $c$ can be found in Moroni et al. (1995) instead of the values used by Gross and Kohn (1985)] and $\lim _{q \rightarrow \infty} f_{x c}(q, \omega \neq 0)$ $=-\left(8 \pi / 3 q^{2}\right)[1-g(0)]$, where $g(0)$ is the paircorrelation function evaluated at zero distance;

(iv) The following relations satisfied in the highfrequency limit by the real and imaginary parts of $f_{x c}$ for $q<\infty: \lim _{\omega \rightarrow \infty} \operatorname{Re} f_{x c}(q, \omega)=f_{x c}(0, \infty)+c / \omega^{3 / 2}$ and $\lim _{\omega \rightarrow \infty} \operatorname{Im} f_{x c}(q, \omega)=-c / \omega^{3 / 2}$, with $c=23 \pi / 15$ in the high-density limit evaluation of the irreducible polarization propagator.

These exact relations were used by Gross and Kohn (1985) to build a Padé approximation to the $f_{x c}(q$ $\rightarrow 0, \omega)$. Although these results were obtained for the three-dimensional electron-gas case, similar relations were obtained for the two-dimensional case (Iwamoto, 1984; Holas and Singwi, 1989) and extended to include two electron-hole pairs in both the longitudinal and the transverse response functions (Nifosí et al., 1998).
Higher exact sum rules (up to seventh order) have been derived by Sturm (1995), and they can be used as a stringent test on the quality of the response functions derived from approximated $f_{x c}$ functionals. ${ }^{50}$

Additional constraints on the matrix elements of the exchange-correlation kernel were obtained by Gonze and Scheffler (1999) using the Keldysh formalism adopted by van Leeuwen (1998). In particular, at the exact exchange level the following exact relation has to be satisfied close to the Kohn-Sham resonance energy $\omega_{i j}$ :

$$
\begin{aligned}
\left\langle\Phi_{i j}\left|f_{x c}\left(\omega_{i j}\right)\right| \Phi_{i j}\right\rangle= & \left\langle\psi_{i}\left|\Sigma_{x}-V_{x}\right| \psi_{i}\right\rangle \\
& -\left\langle\psi_{j}\left|\Sigma_{x}-V_{x}\right| \psi_{j}\right\rangle-\left\langle n_{j}|V| n_{i}\right\rangle,
\end{aligned}
$$

where $v_{x}^{H F}$ is the Hartree-Fock nonlocal operator evaluated with the Kohn-Sham wave functions and $V_{x}$ is the exact exchange potential in DFT. The last term describes an unscreened electron-hole attraction in contrast to the effective interaction in the TDLDA approximation (as discussed above). This is due to the fact that Eq. (A5) for the kernel explicitly shows two parts, namely, the first two contributions containing a shift linked to Hartree-Fock electron addition and removal energies, and the second one correcting with respect to those energies, whereas the TDLDA kernel never passes explicitly through electron addition and removal energies. In the single-pole approximation, expression (A5) leads to a correction of the excitation energies with respect to Kohn-Sham eigenvalue differences, which turns out to be identical to the correction obtained by Görling (1996) in a first-order perturbation theory, in the difference between the many-body and the second-quantized KohnSham Hamiltonians. In addition, the inclusion of correlation effects leads to dynamically screened matrix elements through the same unscreened exchangelike term and an additional screened Coulomb interaction (Gonze and Scheffler, 1999). The similarities of this approach to the $G W$ quasiparticle correction and the inclusion of electron-hole (excitonic) effects within the Bethe-Salpeter equation of many-body theory are described in Sec. VI.

Another rigorous constraint is known as the harmonic-potential theorem (Kohn, 1961; Dobson, 1994). It deals with the motion of an interacting manyelectron system confined in a parabolic potential well under a spatially uniform time-dependent external field. This system exhibits sharp resonances at the bare harmonic-oscillator frequency, independently of the electron-electron interaction. This exact result for the response of an interacting electron system stems from the invariance of the harmonic potential under a transformation to a homogeneously accelerated reference frame (Vignale, 1995). In this case the dynamics of the electronic center of mass is completely decoupled from

\footnotetext{
${ }^{50}$ In particular, the TDLDA and RPA satisfy the first three odd-frequency sum rules but not the seventh (Sturm, 1995).
} 
that of the internal degrees of freedom. This puts interesting constraints on approximate theories of timedependent many-body physics such as TDDFT. Note that the TDLDA approximation to TDDFT satisfies the harmonic-potential theorem because the exchangecorrelation potential follows the density when the latter is moved (that is, the exchange-correlation potential is local in both time and space; see below). The Gross and Kohn (1985) approximation violates the harmonicpotential theorem as shown by Dobson (1994), but it is possible to perform a simple modification for frequencydependent local theories to satisfy this theorem (Vignale and Kohn, 1996; Dobson, Bünner, and Gross, 1997; Vignale et al., 1997). The essential idea is to make the action functional depend on the relative density $\rho_{\text {rel }}(\mathbf{r}, t) \equiv \rho(\mathbf{r}$ $\left.+R_{C M}(t), t\right)$, where $R_{C M}(t)$ is the time evolution of the center of mass of the system. In this spirit an approximate frequency-dependent exchange-correlation functional for small displacements from the equilibrium density $\rho_{0}$ can be constructed, as was done earlier by Gross and Kohn (1985), but now satisfying the harmonicpotential theorem. Thus the exchange-correlation potential reads $V_{x c}(\mathbf{r}, t)=V_{x c}^{L D A}(\mathbf{r})+\int_{t_{0}}^{t} d t^{\prime} f_{x c}\left[\rho_{0}(\mathbf{r}), t\right.$ $\left.-t^{\prime}\right] \delta \rho_{r e l}\left(\mathbf{r}, t^{\prime}\right)$, with $f_{x c}$ as given in Gross and Kohn (1985). ${ }^{51}$

From the previous general relations for $f_{x c}$ one can see that a local-density approximation for timedependent linear response in general does not exist, as long as one keeps on describing dynamical exchangecorrelation effects in terms of the density only. This means that $f_{x c}$ is a strongly nonlocal functional of the density. This problem can be overcome if the current density is added as a basic variable in a generalized Kohn-Sham scheme (Vignale and Kohn, 1996). In this way one can derive a local current-density-functional theory of both current and density responses valid for slowly varying densities and potentials. This scheme was generalized by Vignale et al. (1997) to include dynamical contributions beyond the linear response. In particular, exchange and correlation beyond the TDLDA lead to the appearance of complex and frequency-dependent viscoelasticity stresses that, in the homogeneous electron-gas case, provide an additional damping mechanism to the decay into electron-hole pairs.

The fact that the kernel is very nonlocal is also confirmed by the calculation of the correlation energy of the

\footnotetext{
${ }^{51} \mathrm{~A}$ different perspective in functional development is achieved by the fact that the virial theorem for the exchangecorrelation potential has been shown to hold for timedependent electronic systems (Hessler et al., 1999). Moreover, the time dependence of the exchange-correlation energy is solely determined by the exchange-correlation potential $\left[\partial E_{x c} / \partial t=\int d r \partial \rho(\mathbf{r}, t) / \partial t V_{x c}(\mathbf{r} t)\right]$. These exact relations have important implications for the construction of approximate time-dependent functionals. In particular, the TDLDA approximation is shown to fail badly in regions where the timedependent density differs considerably from its ground-state counterpart (Hessler et al., 1999).
}

homogeneous electron gas by Lein et al. (2000). They have shown that the nonzero spatial range of $f_{x c}\left(\mathbf{r}, \mathbf{r}^{\prime}, \omega\right)$ cannot be neglected, whereas the frequency dependence is less important as far as total correlation energies are concerned. In fact, Gonze et al. (1997) have indicated that $f_{x c}$ must have a $1 / q^{2}$ divergence, for $q \rightarrow 0$. This contribution is supposed to solve the "metal-insulator paradox," i.e., the seeming contradiction concerning systems that are in reality nonmetallic, but have a vanishing Kohn-Sham gap.

\section{APPENDIX B: DERIVATION OF THE EQUATIONS COMMON TO THE TDDFT AND BETHE-SALPETER APPROACHES}

1. Dyson-like equation for the macroscopic dielectric function

Given a matrix of the form

$$
M=\left(\begin{array}{cc}
m_{00} & m_{1}^{T} \\
m_{2} & m
\end{array}\right)
$$

with $m_{00}$ being a $c$ number, its inverse is

$$
\begin{aligned}
M^{-1}= & \left(\begin{array}{cc}
0 & 0 \\
0 & m^{-1}
\end{array}\right)+\frac{1}{\left(m_{00}-m_{1}^{T} m^{-1} m_{2}\right)} \\
& \times\left(\begin{array}{cc}
1 & -m_{1}^{T} m^{-1} \\
-m^{-1} m_{2} & m^{-1} m_{2} m_{1}^{T} m^{-1}
\end{array}\right) .
\end{aligned}
$$

If $M$ is the dielectric function $\varepsilon=1-v P$ in its matrix form in reciprocal space $\left(\mathbf{G}, \mathbf{G}^{\prime}\right)$, then

$$
\varepsilon_{M}=\frac{1}{\varepsilon_{00}^{-1}}=\left[\varepsilon_{00}-\varepsilon_{1}^{T} \epsilon^{-1} \varepsilon_{2}\right]
$$

The quantities $\varepsilon_{00}, \varepsilon_{1}^{T}, \varepsilon_{2}$, and $\epsilon$ are given by

$$
\begin{aligned}
& \varepsilon_{00}=1-v_{0} P_{00}, \\
& {\left[\varepsilon_{1}^{T}\right]_{\mathbf{G}}=-v_{0} P_{0 \mathbf{G}}, \quad \mathbf{G} \neq 0,} \\
& {\left[\varepsilon_{2}\right]_{\mathbf{G}^{\prime}}=-v_{\mathbf{G}^{\prime}} P_{\mathbf{G}^{\prime} 0}, \quad \mathbf{G}^{\prime} \neq 0,} \\
& {[\boldsymbol{\epsilon}]_{\mathbf{G G}^{\prime}}=\delta_{\mathbf{G G}^{\prime}}-v_{\mathbf{G}} P_{\mathbf{G G}^{\prime}}, \quad \mathbf{G}, \mathbf{G}^{\prime} \neq 0 .}
\end{aligned}
$$

This yields

$$
\varepsilon_{M}=1-v_{0} P_{00}-\sum_{\mathbf{G}, \mathbf{G}^{\prime} \neq 0} v_{0} P_{0 \mathbf{G}} \epsilon_{\mathbf{G G}}^{-1} v_{\mathbf{G}^{\prime}} P_{\mathbf{G}^{\prime} 0} .
$$

Now one defines

$$
\bar{\varepsilon}_{\mathbf{G G}^{\prime}} \equiv \delta_{\mathbf{G}, \mathbf{G}^{\prime}}-\bar{v}_{\mathbf{G}} P_{\mathbf{G G}^{\prime}}
$$

with

$$
\bar{v}_{\mathbf{G}} \equiv\left\{\begin{array}{cc}
0, & \mathbf{G}=0 \\
v_{\mathbf{G}}, & \mathbf{G} \neq 0
\end{array}\right.
$$

i.e., $\bar{v}$ indicates that the $\mathbf{G}=0$ contribution is to be left out in the bare Coulomb interaction. In matrix notation this yields

$$
\bar{\varepsilon}_{\mathbf{G G}^{\prime}}=\left(\begin{array}{cc}
1 & 0^{T} \\
\boldsymbol{\varepsilon}_{2} & \boldsymbol{\epsilon}
\end{array}\right)
$$


Then the inverse of $\bar{\varepsilon}$ is given by using Eq. (B2),

$$
\bar{\varepsilon}_{\mathbf{G} \mathbf{G}^{\prime}}^{-1}=\left(\begin{array}{cc}
1 & 0^{T} \\
-\epsilon^{-1} \varepsilon_{2} & \epsilon^{-1}
\end{array}\right) .
$$

Thus Eq. (B8) leads to Eq. (2.9), if in the latter $\bar{P}$ is defined as

$$
\bar{P}_{\mathbf{G G}^{\prime}} \equiv P_{\mathbf{G G}^{\prime}}+\sum_{K, K^{\prime}} P_{\mathbf{G} K^{\prime}} \bar{\varepsilon}_{K K^{\prime}}^{-1} \bar{v}_{K^{\prime}}(q) P_{K^{\prime} \mathbf{G}^{\prime}} .
$$

This gives the wanted matrix expression,

$$
\bar{P}=P+P \bar{\varepsilon}^{-1} \bar{v} P,
$$

and, together with $\bar{\varepsilon}^{-1}=(1-\bar{v} P)^{-1}$, the Dyson-like equation (2.10).

\section{Effective two-particle equations}

In order to solve a Dyson-like equation such as Eq. (2.20), one has to invert a four-point function for each frequency. This problem can be reformulated as an effective eigenvalue problem; in fact, the physical picture of interacting electron-hole pairs suggests using a basis of eigenfunctions $\psi_{n}$ of the effective one-particle Hamiltonian, from which the starting density matrix had been constructed, expecting that only a limited number of electron-hole pairs will contribute to each excitation (see Fetter and Walecka, 1971). These functions are supposed to form an orthonormal and complete set. Any four-point function $S$ can then be transformed as

$$
\begin{aligned}
& S\left(\mathbf{r}_{1}, \mathbf{r}_{1} ; \mathbf{r}_{2}^{\prime}, \mathbf{r}_{2}^{\prime}\right) \\
& \quad=\sum_{n_{1} \cdots} \psi_{n_{4}}^{*}\left(\mathbf{r}_{1}\right) \psi_{n_{2}}\left(\mathbf{r}_{1}^{\prime}\right) \psi_{n_{3}}\left(\mathbf{r}_{2}\right) \psi_{n_{4}}^{*}\left(\mathbf{r}_{2}^{\prime}\right) S_{\left(n_{1} n_{2}\right)\left(n_{3} n_{4}\right)} .
\end{aligned}
$$

The four-point independent-quasiparticle polarization ${ }^{4} P_{I Q P}$,

$$
\begin{aligned}
& { }^{4} P_{I Q P}\left(\mathbf{r}_{1}, \mathbf{r}_{2}, \mathbf{r}_{3}, \mathbf{r}_{4}\right) \\
& =\sum_{n, n^{\prime}} \frac{\left(f_{n}-f_{n^{\prime}}\right) \psi_{n}^{*}\left(\mathbf{r}_{1}\right) \psi_{n^{\prime}}\left(\mathbf{r}_{2}\right) \psi_{n^{\prime}}^{*}\left(\mathbf{r}_{4}\right) \psi_{n}\left(\mathbf{r}_{3}\right)}{\epsilon_{n}-\epsilon_{n^{\prime}}-\omega},
\end{aligned}
$$

is then diagonal in this basis and reads ${ }^{4} P_{I Q P\left(n_{1} n_{2}\right)\left(n_{3} n_{4}\right)}=\left(f_{n_{2}}-f_{n_{1}}\right) \delta_{n_{1}, n_{3}} \delta_{n_{2}, n_{4}} /\left(\epsilon_{n_{2}}\right.$ $\left.-\epsilon_{n_{1}}-\omega\right)$. With the definition $\bar{\Pi} \equiv\left[1-{ }^{4} P_{I Q P} K\right]^{-1}$, Eq. (2.20) becomes $S=\bar{\Pi}^{4} P_{I Q P}$. In transition space,

$$
\begin{aligned}
\Pi_{\left(n_{1} n_{2}\right)\left(n_{3} n_{4}\right)}= & {\left[\left(\epsilon_{m_{2}}-\boldsymbol{\epsilon}_{m_{1}}-\boldsymbol{\omega}\right) \delta_{m_{1}, m_{3}} \delta_{m_{2}, m_{4}}\right.} \\
& \left.+\left(f_{m_{1}}-f_{m_{2}}\right) K_{\left(m_{1} m_{2}\right)\left(m_{3} m_{4}\right)}\right]_{\left(n_{1} n_{2}\right)\left(n_{3} n_{4}\right)}^{-1} \\
& \times\left(\epsilon_{n_{4}}-\epsilon_{n_{3}}-\omega\right) .
\end{aligned}
$$

Defining an effective two-particle Hamiltonian,

$$
\begin{aligned}
H_{\left(n_{1} n_{2}\right)\left(n_{3} n_{4}\right)}^{2 p} \equiv & \left(\epsilon_{n_{2}}-\epsilon_{n_{1}}\right) \delta_{n_{1}, n_{3}} \delta_{n_{2}, n_{4}} \\
& +\left(f_{n_{1}}-f_{n_{2}}\right) K_{\left(n_{1} n_{2}\right)\left(n_{3} n_{4}\right)},
\end{aligned}
$$

one can then rewrite $S$ as

$$
S_{\left(n_{1} n_{2}\right)\left(n_{3} n_{4}\right)}=\left[H^{2 p}-I \omega\right]_{\left(n_{1} n_{2}\right)\left(n_{3} n_{4}\right)}^{-1}\left(f_{n_{4}}-f_{n_{3}}\right) .
$$

The one-particle transition energies on the diagonal are the eigenvalues of the starting one-particle Hamiltonian. Schematically, the effective Hamiltonian [Eq. (B17)] has the form

$$
H_{\left(n_{1} n_{2}\right)\left(n_{3} n_{4}\right)}^{2 p}=\left(\begin{array}{cc}
A & B \\
0 & D
\end{array}\right),
$$

with

$$
A=\begin{array}{c|cc}
\left(n_{1} n_{2}\right) \downarrow & & \\
\hline\left(n_{3} n_{4}\right) \rightarrow & \left\{v^{\prime} c^{\prime}\right\} & \left\{c^{\prime} v^{\prime}\right\} \\
\hline\{v c\} & H_{(v c)\left(v^{\prime} c^{\prime}\right)}^{2 p, r e s} & K_{(v c)\left(c^{\prime} v^{\prime}\right)}
\end{array},
$$

$$
B=\begin{array}{c|cc} 
& \left\{v^{\prime} \tilde{v}^{\prime}\right\} & \left\{c^{\prime} \tilde{c}^{\prime}\right\} \\
\hline\{v c\} & K_{(v c)\left(v^{\prime} \tilde{v}^{\prime}\right)} & K_{(v c)\left(c^{\prime} \bar{c}^{\prime}\right)}
\end{array},
$$

$$
D=\begin{array}{c|cc} 
& \left\{v^{\prime} \tilde{v}^{\prime}\right\} & \left\{c^{\prime} \tilde{c}^{\prime}\right\} \\
\hline\{v \tilde{v}\} & \left(\epsilon_{\tilde{v}}-\epsilon_{v}\right) \delta_{v v^{\prime}} \delta_{\tilde{v} \tilde{v}^{\prime}} & 0 \\
\{c \tilde{c}\} & 0 & \left(\epsilon_{\tilde{c}}-\epsilon_{c}\right) \delta_{c c^{\prime}} \delta_{\tilde{c} \tilde{c}^{\prime}}
\end{array}
$$

The resonant part is defined as

$$
H_{(v c)\left(v^{\prime} c^{\prime}\right)}^{2 p, r e s} \equiv\left(\epsilon_{c}-\epsilon_{v}\right) \delta_{v, v^{\prime}} \delta_{c, c^{\prime}}+K_{(v c)\left(v^{\prime} c^{\prime}\right)} .
$$

It corresponds to transitions at positive absorption frequencies $\omega$. Due to the factor $\left(f_{n_{3}}-f_{n_{4}}\right)$ in Eq. (B18), only the first column of

$$
M_{\left(n_{1} n_{2}\right)\left(n_{3} n_{4}\right)}^{-1}=\left[H^{2 p}-I \omega\right]_{\left(n_{1} n_{2}\right)\left(n_{3} n_{4}\right)}^{-1},
$$

i.e., with $\left(n_{3} n_{4}\right)=\left\{c^{\prime} v^{\prime}\right\}$ and $\left\{v^{\prime} c^{\prime}\right\}$, contributes to the calculation of $S$.

Defining $A_{\omega}=A-I \omega$, it is clear that only $A_{\omega}^{-1}$ is going to be relevant, in other words, the part $A$ in Eq. (B20). This means that only pairs containing one filled and one empty Bloch state contribute, which is physically meaningful. It reflects the fact that only the hole-particle part [Eq. (3.7)] of the two-particle Green's function yields the absorption and loss spectra, as pointed out in Sec. III.A.

It is this part $A$ in Eq. (B20) which is referred to as the two-particle Hamiltonian $H^{2 p}$ in the present work. It is in general not Hermitian and can be further separated into four blocks: two blocks on the diagonal with the transition energies and the interaction kernel $K$, and two off-diagonal coupling blocks with contributions only from the interaction kernel: 


$$
H^{2 p}=\left(\begin{array}{cc}
H^{2 p, \text { res }} & H^{\text {coupling }} \\
-\left[H^{\text {coupling }}\right]^{*} & -\left[H^{2 p, r e s}\right]^{*}
\end{array}\right) .
$$

The resonant part is Hermitian, $\left(H^{2 p, r e s}\right) *$ $=\left(H^{2 p, r e s}\right)^{T}$, while the coupling part alone is symmetric, $H^{\text {coupling }}=\left(H^{\text {coupling }}\right)^{T}$. The part in the lower right is denoted antiresonant. Neglecting the coupling part is called the Tamm-Dancoff approximation.

The spectral representation of the inverse two-particle Hamiltonian is

$$
\left[H^{2 p}-I \omega\right]_{\left(n_{1} n_{2}\right)\left(n_{3} n_{4}\right)}^{-1}=\sum_{\lambda, \lambda^{\prime}} \frac{A_{\lambda}^{\left(n_{1} n_{2}\right)} N_{\lambda, \lambda^{\prime}}^{-1} A_{\lambda^{\prime}}^{*\left(n_{3} n_{4}\right)}}{E_{\lambda}-\omega}
$$

which holds for a system of eigenvectors and eigenvalues of a general (not necessarily Hermitian) matrix defined by $H^{2 p} A_{\lambda}=E_{\lambda} A_{\lambda}$, where $N_{\lambda, \lambda^{\prime}}$ $\equiv \Sigma_{n_{1} n_{2}} A_{\lambda}^{*\left(n_{1} n_{2}\right)} A_{\lambda^{\prime}}^{\left(n_{1} n_{2}\right)}$ is the overlap matrix of the generally nonorthogonal eigenstates of $H^{2 p}$. Hence $H^{2 p}$ is diagonalized, and from its eigenvalues $E_{\lambda}$ and eigenstates $A_{\lambda}^{n_{1} n_{2}}$ the four- and two-point quantities of interest are constructed. In particular, one obtains the macroscopic dielectric function given in Eq. (2.23), and an equivalent expression for $\chi$.

\section{APPENDIX C: AN $f_{x c}$ FROM THE BETHE-SALPETER APPROACH}

In this subsection, we derive a time-dependent density-functional formalism for excitation spectra from the many-body Bethe-Salpeter equation (Reining et al., 2002). The results obtained using an approximation to this kernel, denoted as RORO, were already discussed in Sec. V.C.

The main idea is to compare the effective two-particle Hamiltonians $H^{2 p, T D D F T}$ and $H^{2 p, B S E}$ derived for TDDFT and the Bethe-Salpeter equation, respectively. If the quasiparticle and Kohn-Sham eigenfunctions are equal, and if all matrix elements of $H^{2 p, T D D F T}$ and $H^{2 p, B S E}$ involving those transitions which actually contribute to the spectrum are equal, than the resulting spectra will be identical. This yields the condition

$$
\begin{aligned}
f_{x c}\left(\mathbf{q}, \mathbf{G}, \mathbf{G}^{\prime}\right)= & \sum_{n_{1} n_{2} n_{3} n_{4}} \frac{1}{\left(f_{n_{1}}-f_{n_{2}}\right)} \Phi^{-1}\left(n_{1}, n_{2} ; \mathbf{G}\right) \\
& \times \mathcal{F}_{\left(n_{1} n_{2}\right)\left(n_{3} n_{4}\right)}\left(\Phi^{*}\right)^{-1}\left(n_{3}, n_{4} ; \mathbf{G}^{\prime}\right) .
\end{aligned}
$$

The factor $\left(f_{n_{1}}-f_{n_{2}}\right)$ can never be zero for the particlehole and hole-particle contributions of a nonmetal. The matrices $\Phi$ are defined as

$$
\Phi\left(n_{1}, n_{2}, \mathbf{r}\right):=\psi_{n_{1}}(\mathbf{r}) \psi_{n_{2}}^{*}(\mathbf{r})
$$

and the operator $\mathcal{F}$ is

$$
\begin{aligned}
\mathcal{F}_{\left(n_{1} n_{2}\right)\left(n_{3} n_{4}\right)}= & \left(\epsilon_{n_{2}}^{\mathrm{QP}}-\epsilon_{n_{1}}^{\mathrm{QP}}-\epsilon_{n_{2}}^{\mathrm{DFT}}+\epsilon_{n_{1}}^{\mathrm{DFT}}\right) \delta_{n_{1} n_{3}} \delta_{n_{2} n_{4}} \\
& -\left(f_{n_{1}}-f_{n_{2}}\right) W_{\left(n_{1} n_{2}\right)\left(n_{3} n_{4}\right)}
\end{aligned}
$$

It is now clear that, if the transformation from real to transition space $x_{l} \rightarrow \psi_{n}\left(x_{l}\right)$ was complete in all four indices, Eq. (B27) could never be satisfied, since otherwise $H^{2 p, T D D F T}$ and $H^{2 p, B S E}$ should also be equal in real space-and they cannot be, due to the way the $\delta$ functions are put. On the other hand, if the two operators cannot be made equal, then the spectra can be equal only if at least one of the two operators (in that case, $f_{x c}$ ) is energy dependent. However, in practice only a finite number of transitions contribute to the optical spectrum. This means that one can use an incomplete basis in transition space. Therefore one can still find a static operator that satisfies the required equality in transition space in a particular energy range, even though the real-space operators are not equal. (The invertibility of the matrices $\Phi$ may, however, be questionable in some particular cases).

In view of the ongoing discussions about the exchange-correlation kernel it is interesting to examine some of its features, and in particular its long-range behavior for solids. To this end, we note that $\Phi_{v, c, \mathbf{k}+\mathbf{q}, \mathbf{k}}(\mathbf{G}=0)$ goes to zero as $\mathbf{q}$ for small $q$. Since $W_{v c, v c}$ behaves as a constant, if $\Phi$ is invertible this implies immediately that $f_{x c}\left(\mathbf{q}, \mathbf{G}=\mathbf{G}^{\prime}=0\right)$ behaves as $1 / q^{2}$. Note that there are two such long-range terms coming from (a) the electron-hole attraction (of negative sign) and (b) the energy shift between the quasiparticle and the DFT eigenvalues [of positive sign, as predicted by Gonze et al., 1997, on the basis of their study of the polarization-dependence of the exchangecorrelation energy (Gonze et al., 1995; Resta, 1996), and as verified by a comparison of Kohn-Sham and experimental linear and nonlinear susceptibilities by Aulbur et al., 1996]. A discussion of how this approximated kernel reproduces the optical spectrum of semiconductors for the case of bulk silicon is presented in Sec. V.C.

\section{REFERENCES}

Abrikosov, A. A., L. P. Gorkov, and I. E. Dzyaloshinski, 1963, Methods of Quantum Field Theory in Statistical Physics (Prentice-Hall, Englewood Cliffs, NJ).

Adler, S. L., 1962, Phys. Rev. 126, 413.

Adler, S. L., 1963, Phys. Rev. 130, 1654.

Albrecht, S., G. Onida, and L. Reining, 1997, Phys. Rev. B 55, 10278.

Albrecht, S., L. Reining, R. Del Sole, and G. Onida, 1998a, Phys. Rev. Lett. 80, 4510.

Albrecht, S., L. Reining, R. Del Sole, and G. Onida, 1998b, Phys. Status Solidi A 170, 189.

Albrecht, S., L. Reining, V. Olevano, G. Onida, and R. Del Sole, 1999, Phys. Rev. Lett. 83, 3971.

Almbladh, C.-O., and L. Hedin, 1983, in Handbook on Synchrotron Radiation, edited by E. E. Koch (North-Holland, Amsterdam), Vol. 1, p. 607.

Almbladh, C.-O., and U. von Barth, 1985, Phys. Rev. B 31, 3231.

Anisimov, V. I., F. Aryasetiawan, and A. I. Lichtenstein, 1997, J. Phys.: Condens. Matter 9, 767.

Arnaud, B., and M. Alouani, 2000, Phys. Rev. B 62, 4464.

Arnaud, B., and M. Alouani, 2001, Phys. Rev. B 63, 085208. 
Aryasetiawan, F., 1992, Phys. Rev. B 46, 13051.

Aryasetiawan, F., 1994, Phys. Rev. B 50, 7311.

Aryasetiawan, F., and O. Gunnarsson, 1995, Phys. Rev. Lett.

74, 3221.

Aryasetiawan, F., and O. Gunnarsson, 1998, Rep. Prog. Phys. 61, 237.

Aryasetiawan, F., O. Gunnarsson, and A. Rubio, 2002a, Europhys. Lett. 57, 683.

Aryasetiawan, F., L. Hedin, and K. Karlsson, 1996, Phys. Rev. Lett. 77, 2268.

Aryasetiawan, F., and K. Karlsson, 1994, Phys. Rev. Lett. 73, 1679.

Aryasetiawan, F., T. Miyake, and K. Terakura, 2002b, Phys. Rev. Lett. 88, 166401.

Aulbur, W. G., L. Jönsson, and J. W. Wilkins, 1996, Phys. Rev. B 54, 8540 .

Aulbur, W. G., L. Jönsson, and J. W. Wilkins, 1999, Solid State Phys. 54, 1.

Aulbur, W. G., M. Städele, and A. Görling, 2000, Phys. Rev. B 62, 7121.

Baerends, E. J., 2001, Phys. Rev. Lett. 87, 133004.

Baroni, S., 1984, J. Chem. Phys. 80, 5703.

Baroni, S., S. de Gironcoli, A. Dal Corso, and P. Giannozzi, 2001, Rev. Mod. Phys. 73, 515.

Bassani, F., and G. Pastori Parravicini, 1975, in Electronic States and Optical Transitions in Solids, edited by R. A. Ballinger (Pergamon, Oxford/New York/Toronto), p. 177.

Bauernschmitt, R., and R. Ahlrichs, 1996, Chem. Phys. Lett. 256, 454.

Baym, G., 1962, Phys. Rev. 127, 1391.

Baym, G., and L. P. Kadanoff, 1961, Phys. Rev. 124, 287.

Bechstedt, F., 1992, Adv. Solid State Phys. 32, 161.

Bechstedt, F., R. Del Sole, G. Cappellini, and L. Reining, 1992, Solid State Commun. 84, 765.

Bechstedt, F., M. Fiedler, C. Kress, and R. Del Sole, 1994, Phys. Rev. B 49, 7357.

Bechstedt, F., K. Tenelsen, B. Adolph, and R. Del Sole, 1997, Phys. Rev. Lett. 78, 1528.

Benedict, L. X., and E. L. Shirley, 1999, Phys. Rev. B 59, 5441. Benedict, L. X., E. L. Shirley, and R. B. Bohn, 1998a, Phys. Rev. Lett. 80, 4514.

Benedict, L. X., E. Shirley, and R. B. Bohn, 1998b, Phys. Rev. B 57, R9385.

Bennett, M., and J. C. Inkson, 1977, J. Phys. C 10, 987.

Bertsch, G. F., and R. A. Broglia, 1994, Oscillations in Finite Quantum Systems (Cambridge University Press, Cambridge, England).

Bertsch, G. F., J.-I. Iwata, A. Rubio, and K. Yabana, 2000, Phys. Rev. B 62, 7998.

Bertsch, G. F., A. Rubio, and K. Yabana, 2000, preprint physics/0003090.

Bertsch, G. F., and S. F. Tsai, 1975, Phys. Rep. 18, 125.

Blase, X., A. Rubio, S. G. Louie, and M. L. Cohen, 1995, Phys. Rev. B 52, R2225.

Blöchl, P. E., 1994, Phys. Rev. B 50, 17953.

Bonacić-Koutecký, V., P. Fantucci, and J. Koutecky, 1990, J. Chem. Phys. 93, 3802.

Botti, S., F. Sottile, N. Vast, V. Olevano, G. Onida, A. Rubio, and L. Reining, 2002, unpublished.

Brener, N. E., 1975a, Phys. Rev. B 11, 929.

Brener, N. E., 1975b, Phys. Rev. B 11, 1600.

Brinkman, W., and B. Goodman, 1966, Phys. Rev. 149, 597.
Bürgi, L., O. Jeandupeux, H. Brune, and K. Kern, 1999, Phys. Rev. Lett. 82, 4516.

Burke, K., M. Petersilka, and E. K. U. Gross, 2002, in Recent Advances in Density Functional Methods, Vol. III, edited by P. Fantucci and A. Bencini (World Scientific, Singapore).

Cai, Z.-L., D. J. Tozer, and J. R. Reimers, 2000, J. Chem. Phys. 113, 7084.

Caliebe, W. A., J. A. Soininen, E. L. Shirley, C.-C. Kao, and K. Hämäläinen, 2000, Phys. Rev. Lett. 84, 3907.

Campillo, I., J. M. Pitarke, A. Rubio, E. Zarate, and P. M. Echenique, 1999, Phys. Rev. Lett. 83, 2230.

Campillo, I., J. M. Pitarke, A. Rubio, and P. M. Echenique, 2000, Phys. Rev. B 62, 1500.

Campillo, I., A. Rubio, J. M. Pitarke, A. Goldmann, and P. M. Echenique, 2000, Phys. Rev. Lett. 85, 3241.

Campillo, I., V. M. Silkin, J. M. Pitarke, E. V. Chulkov, A. Rubio, and P. M. Echenique, 2000, Phys. Rev. B 61, 13484.

Cardona, M., L. F. Lastras-Martinez, and D. E. Aspnes, 1999, Phys. Rev. Lett. 83, 3970.

Casida, M. E., 1995, in Recent Advances in Density Functional Methods, Part I, edited by D. P. Chong (World Scientific, Singapore), p. 155.

Casida, M. E., 1996, in Recent Developments and Applications of Modern Density Functional Theory, edited by J. M. Seminario (Elsevier Science, Amsterdam), p. 391.

Casida, M. E., F. Gutierrez, J. Guan, F. X. Gadea, D. Salahub, and J. P. Daudey, 2000, J. Chem. Phys. 113, 7062.

Casida, M. E., and D. R. Salahub, 2000, J. Chem. Phys. 113, 8918.

Castro, A., M. A. L. Marques, J. A. Alonso, G. F. Bertsch, K. Yabana, and A. Rubio, 2001, J. Chem. Phys. 118, 1930.

Cazalilla, M. A., J. S. Dolado, A. Rubio, and P. M. Echenique, 2000, Phys. Rev. B 61, 8033.

Cepek, C., I. Vobornik, A. Goldoni, E. Magnano, G. Selvaggi, J. Kröger, G. Panaccione, G. Rossi, and M. Sancrotti, 2001, Phys. Rev. Lett. 86, 3100.

Ceperley, D. M., and B. J. Alder, 1980, Phys. Rev. Lett. 45, 566. Chang, E. K., M. Rohlfing, and S. G. Louie, 2000, Phys. Rev. Lett. 85, 2613.

Charlesworth, J. P. A., R. W. Godby, and R. J. Needs, 1993, Phys. Rev. Lett. 70, 1685.

Cini, M., 1977, Solid State Commun. 24, 681.

Cini, M., 1979, Surf. Sci. 87, 483.

Cini, M., and C. Verdozzi, 1986, Solid State Commun. 57, 657. Cohen, M. L., and J. R. Chelikowsky, 1988, Electronic Structure and Optical Properties of Semiconductors, Solid-State Sciences No. 75 (Springer, New York).

Corradini, M., R. Del Sole, G. Onida, and M. Palummo, 1998, Phys. Rev. B 57, 14569.

Courths, R., and S. Hüfner, 1984, Phys. Rep. 112, 53.

de Boeij, P. L., F. Koostra, J. A. Berger, R. van Leeuwen, and J. G. Sniders, 2001, J. Chem. Phys. 115, 1995.

de Groot, H. J., R. T. M. Ummels, P. A. Bobbert, and W. van Haeringen, 1996, Phys. Rev. B 54, 2374.

del Castillo-Mussot, M., and L. J. Sham, 1985, Phys. Rev. B 31, 2092.

Del Sole, R., and R. Girlanda, 1993, Phys. Rev. B 48, 11789.

Del Sole, R., L. Reining, and R. W. Godby, 1994, Phys. Rev. B 49, 8024.

Del Sole, R., and A. Selloni, 1984, Phys. Rev. B 30, 883.

Diau, E. W.-G., J. L. Herek, Z. H. Kim, and A. H. Zewail, 1998, Science 279, 847.

Dobson, J. F., 1994, Phys. Rev. Lett. 73, 2244. 
Dobson, J. F., M. J. Bünner, and E. K. U. Gross, 1997, Phys. Rev. Lett. 79, 1905.

Dobson, J. F., G. H. Harris, and A. J. O'Connor, 1988, J. Phys.: Condens. Matter 2, 6461.

Dobson, J., G. Vignale, and M. P. Das, 1997, Eds., Electronic Density Functional Theory: Recent Progress and New Directions (Plenum, New York).

Dreizler, R. M., and E. K. U. Gross, 1990, Density Functional Theory: An Approach to the Quantum Many-Body Problem (Springer, Berlin).

DuBois, D. F., 1959, Ann. Phys. (N.Y.) 7, 174; 8, 24.

Dudarev, S. L., A. I. Liechtenstein, M. R. Castell, G. A. D. Briggs, and A. P. Sutton, 1997, Phys. Rev. B 56, 4900.

Echenique, P. M., J. M. Pitarke, E. Chulkov, and A. Rubio, 2000, Chem. Phys. 251, 1.

Eder, R., H. F. Pen, and G. A. Sawatzky, 1997, Phys. Rev. B 56, 10115.

Ehrenreich, H., 1966, in The Optical Properties of Solids, Proceedings of the International School of Physics "Enrico Fermi," Course XXXIV, edited by J. Tauc (Academic, New York), p. 106.

Ehrenreich, H., and M. H. Cohen, 1959, Phys. Rev. 115, 786.

Ekardt, W. P., and J. M. Pacheco, 1995, Phys. Rev. B 52, 16864. Engel, G. E. and B. Farid, 1993, Phys. Rev. B 47, 15931.

Farid, B., 1999a, in Electron Correlation in the Solid State, edited by N. H. March (World Scientific, Imperial College), p. 103.

Farid, B., 1999b, Philos. Mag. Lett. 79, 581.

Farid, B., 2001, preprint cond-mat/0110481.

Fetter, A. L., and J. D. Walecka, 1971, Quantum Theory of Many-Particle Systems (McGraw-Hill, New York).

Filippi, C., C. J. Umrigar, and X. Gonze, 1997, J. Chem. Phys. 107, 9994.

Fleszar, A., and W. Hanke, 2000, Phys. Rev. B 62, 2466.

Fleszar, A., A. A. Quong, and A. G. Eguiluz, 1995, Phys. Rev. Lett. 74, 590.

Fleszar, A., R. Stumpf, and A. G. Eguiluz, 1997, Phys. Rev. B 55, 2068.

Fock, V., 1930, Z. Phys. 61, 126.

Frenkel, J., 1931a, Phys. Rev. 37, 17.

Frenkel, J., 1931b, Phys. Rev. 37, 1276.

Galitskii, V. M., and A. B. Migdal, 1958, Sov. Phys. JETP 7, 96. García-González, P., and R. W. Godby, 2001, Phys. Rev. B 63, 075112.

García-González, P., and R. W. Godby, 2002, Phys. Rev. Lett. 88, 056406.

Gavrilenko, V. I., and F. Bechstedt, 1996, Phys. Rev. B 54, 13416.

Gerlach, A., K. Berge, A. Goldmann, I. Campillo, A. Rubio, J. M. Pitarke, and P. M. Echenique, 2001, Phys. Rev. B 64, 085423.

Georges, A., G. Kotliar, W. Krauth, and M. J. Rozenberg, 1996, Rev. Mod. Phys. 68, 13.

Godby, R. W., and R. J. Needs, 1989, Phys. Rev. Lett. 62, 1169. Godby, R. W., M. Schlüter, and L. J. Sham, 1986, Phys. Rev. Lett. 56, 2415.

Godby, R. W., M. Schlüter, and L. J. Sham, 1987, Phys. Rev. B 36, 6497.

Godby, R. W., M. Schlüter, and L. J. Sham, 1988, Phys. Rev. B 37, 10159.

Godby, R. W., and I. D. White, 1998, Phys. Rev. Lett. 80, 3161. Gonze, X., Ph. Ghosez, and R. W. Godby, 1995, Phys. Rev. Lett. 74, 4035.
Gonze, X., Ph. Ghosez, and R. W. Godby, 1997, Phys. Rev. B 56, 12811.

Gonze, X., and M. Scheffler, 1999, Phys. Rev. Lett. 82, 4416.

Görling, A., 1996, Phys. Rev. A 54, 3912.

Görling, A., 1998, Phys. Rev. A 57, 3433.

Görling, A., 1999, Phys. Rev. Lett. 83, 5459.

Görling, A., and M. Levy, 1994, Phys. Rev. A 50, 196.

Grabo, T., T. Kreibich, S. Kurth, and E. K. U. Gross, 2000a, in Strong Coulomb Correlations in Electronic Structure: Beyond the Local Density Approximation, edited by V. I. Anisimov (Gordon \& Breach, Tokyo), pp. 203-211.

Grabo, T., M. Petersilka, and E. K. U. Gross, 2000b, J. Mol. Struct.: THEOCHEM 501, 353.

Gross, E. K. U., F. J. Dobson, and M. Petersilka, 1996, Density Functional Theory (Springer, New York).

Gross, E. K. U., and W. Kohn, 1985, Phys. Rev. Lett. 55, 2850; 57, 923(E).

Gross, E. K. U., C. A. Ullright, and U. J. Grossmann, 1994, in Density Functional Theory, edited by E. K. U. Gross and R. M. Dreizler (Plenum, New York), p. 149.

Grossman, J. C., L. Mitas, and K. Raghavachari, 1995, Phys. Rev. Lett. 75, 3870.

Grossman, J. C., M. Rohlfing, L. Mitas, S. G. Louie, and M. L. Cohen, 2001, Phys. Rev. Lett. 86, 472.

Gunnarsson, O., and K. Schönhammer, 1986, Phys. Rev. Lett. 56, 1968.

Gygi, F., and A. Baldereschi, 1989, Phys. Rev. Lett. 62, 2160.

Hahn, P. H., W. G. Schmidt, and F. Bechstedt, 2002, Phys. Rev. Lett. 88, 016402.

Haken, H., 1956, Nuovo Cimento III, 1230.

Haken, H., 1957, Z. Phys. 147, 323.

Hamada, N., M. Hwang, and A. J. Freeman, 1990, Phys. Rev. B 41, 3620.

Hanke, W., 1978, Adv. Phys. 27, 287.

Hanke, W., H. J. Mattausch, and G. Strinati, 1983, in Electron Correlations in Solids, Molecules, and Atoms, edited by J. T. Devreese and F. Brosens (Plenum, New York), p. 289.

Hanke, W., and L. J. Sham, 1974, Phys. Rev. Lett. 33, 582.

Hanke, W., and L. J. Sham, 1975, Phys. Rev. B 12, 4501.

Hanke, W., and L. J. Sham, 1980, Phys. Rev. B 21, 4656.

Hartree, D. R., 1928, Proc. Cambridge Philos. Soc. 24, 89.

Haydock, R., 1980, Recursive Solution of the Schroedinger Equation, Solid State Physics Vol. 35 (Academic, London), p. 215.

Hedin, L., 1965, Phys. Rev. 139, A796.

Hedin, L., 1980, Phys. Scr. 21, 477.

Hedin, L., 1995, Int. J. Quantum Chem. 56, 445.

Hedin, L., 1999, J. Phys.: Condens. Matter 11, R489.

Hedin, L., and B. I. Lundqvist, 1971, J. Phys. C 4, 2064.

Hedin, L., and S. Lundqvist, 1969, in Solid State Physics, edited

by H. Ehrenreich, F. Seitz, and D. Turnbull (Academic, New York), Vol. 23, p. 1.

Heller, W. R., and A. Marcus, 1951, Phys. Rev. 84, 809.

Hessler, P., J. Park, and K. Burke, 1999, Phys. Rev. Lett. 82, 378.

Hohenberg, P., and W. Kohn, 1964, Phys. Rev. 136, B864.

Holas, A., and K. S. Singwi, 1989, Phys. Rev. B 40, 158.

Holm, B., and F. Aryasetiawan, 2000, Phys. Rev. B 62, 4858.

Holm, B., and U. von Barth, 1998, Phys. Rev. B 57, 2108.

Hong, S., and G. D. Mahan, 1994, Phys. Rev. B 50, 8182.

Hybertsen, M. S., and S. G. Louie, 1985, Phys. Rev. Lett. 55, 1418.

Hybertsen, M. S., and S. G. Louie, 1986, Phys. Rev. B 34, 5390. 
Hybertsen, M. S., and S. G. Louie, 1988, Phys. Rev. B 37, 2733. Ihee, H., V. A. Lobastov, U. M. Gomez, B. M. Godson, R. Srinivasan, C.-Y. Ruan, and A. H. Zewail, 2001, Science 291, 458.

Inkson, J. C., and M. Bennett, 1978, J. Phys. C 11, 2017.

Itoh, U., Y. Toyoshima, and H. Onuki, 1986, J. Chem. Phys. 85, 4867.

Iwamoto, N., 1984, Phys. Rev. A 30, 3289.

Jensen, Frank, 1999, Introduction to Computational Chemistry (Wiley, Chichester, England), Chap. 4.

Jones, R. O., and O. Gunnarsson, 1989, Rev. Mod. Phys. 61, 689.

Jones, R. O., and G. Seifert, 1997, Phys. Rev. Lett. 79, 443.

Kadanoff, L. P., and G. Baym, 1962, Quantum Statistical Mechanics (Benjamin, New York).

Kanamori, J., 1963, Prog. Theor. Phys. 30, 275.

Keldysh, L. V., 1965, Sov. Phys. JETP 20, 1018.

Kevan, S. D., 1992, Angle-Resolved Photoemission: Theory and Current Applications (Elsevier, Amsterdam).

Keyling, R., W.-D. Schöne, and W. Ekardt, 2000, Phys. Rev. B 61, 1670.

Kim, Y. H., M. Städele, and A. Görling, 2002, Int. J. Quantum Chem. (in press)

Knox, R. S., 1963, Theory of Excitons, Solid State Phys. Suppl.

5 (Academic, New York/London), p. 95.

Koch, E. E., and A. Otto, 1972, Chem. Phys. Lett. 12, 476.

Kohn, W., 1961, Phys. Rev. 123, 1242.

Kohn, W., and L. Sham, 1965, Phys. Rev. 140, A1133.

Kootstra, F., P. L. de Boeji, and J. G. Snijders, 2000, Phys. Rev. B 62, 7071.

Kreibich, T., 2000, Ph.D. thesis (University of Würzburg).

Kreibich, T., and E. K. U. Gross, 2001, Phys. Rev. Lett. 86, 2984.

Ku, W., and A. G. Eguiluz, 1999, Phys. Rev. Lett. 82, 2350.

Ku, W., A. G. Eguiluz, and E. W. Plummer, 2000, Phys. Rev. Lett. 85, 2410.

Kwong, N.-H., and M. Bonitz, 2000, Phys. Rev. Lett. 84, 1768.

Kurth, S., M. Marques, M. Luders, and E. K. U. Gross, 1999,

Phys. Rev. Lett. 83, 2628.

Landau, L. D., 1957a, Sov. Phys. JETP 3, 920.

Landau, L. D., 1957b, Sov. Phys. JETP 5, 101.

Landau, L. D., 1959, Sov. Phys. JETP 35, 70.

Landau, L. D., and E. M. Lifschitz, 1980, Statistical Physics Part II (Pergamon, Oxford).

Langhoff, P. W., S. T. Epstein, and M. Karplus, 1972, Rev. Mod. Phys. 44, 602.

Larson, B. C., J. Z. Tischler, E. D. Isaacs, P. Zschack, A. Fleszar, and A. G. Eguiluz, 1996, Phys. Rev. Lett. 77, 1346.

Lautenschlager, P., M. Garriga, L. Viña, and M. Cardona, 1987, Phys. Rev. B 36, 4821.

Lein, M., E. K. U. Gross, and J. P. Perdew, 2000, Phys. Rev. B 61, 13431.

Levine, Z. H., and S. G. Louie, 1982, Phys. Rev. B 25, 6310.

Levine, Z. H., and D. C. Allan, 1989, Phys. Rev. Lett. 63, 1719.

Liebsch, A., 1997, Electronic Excitations at Metal Surfaces (Plenum, New York).

Lindhard, J., 1954, K. Dan. Vidensk. Selsk. Mat. Fys. Medd. 28, 57.

Lipari, N. O., and W. B. Fowler, 1970, Phys. Rev. B 2, 3354.

Louie, S. G., J. Chelikowsky, and M. L. Cohen, 1975, Phys. Rev. Lett. 34, 155.

Lundqvist, B. I., 1967, Phys. Kondens. Mater. 6, 193.

Lundqvist, B. I., 1968, Phys. Kondens. Mater. 7, 117.
Lundqvist, S., and N. H. March, 1983, Eds., Theory of the Inhomogeneous Electron Gas (Plenum, New York).

Mackrodt, W. C., N. M. Harrison, V. R. Saunders, N. L. Allan, and M. D. Towler, 1996, Chem. Phys. Lett. 250, 66.

Maddocks, N. E., R. W. Godby, and R. J. Needs, 1994a, Europhys. Lett. 27, 681.

Maddocks, N. E., R. W. Godby, and R. J. Needs, 1994b, Phys. Rev. B 49, 8502.

Mahan, G. D., 1994, Comments Condens. Matter Phys. 16, 333. Mahan, G. D., and B. E. Sernelius, 1989, Phys. Rev. Lett. 62, 2718.

March, N. H., A. Rubio, and J. A. Alonso, 1999, J. Phys. B 32, 2173.

Marini, A., G. Onida, and R. Del Sole, 2001, Phys. Rev. B 64, 195125.

Marini, A., G. Onida, and R. Del Sole, 2002, Phys. Rev. Lett. 88, 016403 .

Marmorkos, I. K., and S. Das Sarma, 1991, Phys. Rev. B 44, 3451.

Marques, M., A. Castro, and A. Rubio, 2001, J. Chem. Phys. 115, 3006.

Martin, P. C., and J. Schwinger, 1959, Phys. Rev. 115, 1342.

Martins, J. L., J. Buttet, and R. Car, 1985, Phys. Rev. B 31, 1804.

Massidda, S., A. Continenza, M. Posternak, and A. Baldereschi, 1995, Phys. Rev. Lett. 74, 2323.

Mauger, A., and M. Lannoo, 1977, Phys. Rev. B 15, 2324.

McLachlan, A. D., and M. A. Ball, 1964, Rev. Mod. Phys. 36, 844.

Miyake, T., F. Aryasetiawan, H. Kino, and K. Terakura, 2000, Phys. Rev. B 61, 16491.

Moroni, S., D. M. Ceperley, and G. Senatore, 1995, Phys. Rev. Lett. 75, 689.

Nannarone, S., P. Chiaradia, F. Ciccacci, R. Memeo, P. Sassaroli, S. Selci, and G. Chiarotti, 1980, Solid State Commun. 33, 593.

Ng, K. K., F. C. Zhang, V. I. Anisimov, and T. M. Rice, 1997, Phys. Rev. Lett. 78, 1311.

Nifosi, R., S. Conti, and M. P. Tosi, 1998, Phys. Rev. B 58, 12758.

Nolting, F., A. Scholl, J. Stöhr, J. W. Seo, J. Fompeyrine, H. Siegwart, J.-P. Locquet, S. Anders, J. Lüning, E. E. Fullerton, M. F. Toney, M. R. Scheinfein, and H. A. Padmore, 2000, Nature (London) 405, 767.

Oğüt, S., J. R. Chelikowsky, and S. G. Louie, 1997, Phys. Rev. Lett. 79, 1770 .

Oğüt, S., J. R. Chelikowsky, and S. G. Louie, 1998, Phys. Rev. Lett. 80, 3162.

Olevano, V., and L. Reining, 2000, MRS Symposium Proceedings Series No. 579; preprint cond-mat/0010194.

Olevano, V., and L. Reining, 2001a, Phys. Rev. Lett. 86, 5962. Olevano, V., and L. Reining, 2001b, unpublished.

Onida, G., L. Reining, R. W. Godby, R. Del Sole, and W. Andreoni, 1995, Phys. Rev. Lett. 75, 818.

Ortiz, G., M. Harris, and P. Ballone, 1999, Phys. Rev. Lett. 82, 5317.

Pacheco, J. M., and W. P. Ekardt, 1997, Z. Phys. B: Condens. Matter 103, 327.

Peierls, R., 1932, Ann. Phys. (Leipzig) 13, 905.

Perdew, J. P., and A. Zunger, 1981, Phys. Rev. B 23, 5048.

Perdew, J. P., K. Burke, and M. Ernzerhof, 1996, Phys. Rev. Lett. 77, 3865. 
Petek, H., M. J. Weida, H. Nagano, and S. Ogawa, 2000, Science 288, 1402.

Petersilka, M., U. J. Gossmann, and E. K. U. Gross, 1996, Phys. Rev. Lett. 76, 1212.

Petersilka, M., U. J. Gossmann, and E. K. U. Gross, 1998, in Electronic Density Functional Theory: Recent Progress and New Directions, edited by J. F. Dobson, G. Vignale, and M. P. Das (Plenum, New York), p. 177.

Petersilka, M., E. K. U. Gross, and K. Burke, 2000, Int. J. Quantum Chem. 80, 534.

Pines, D., 1963, Elementary Excitations in Solids (AddisonWesley, New York).

Pines, D., and P. Nozières, 1989, The Theory of Quantum Liquids (Addison-Wesley, New York).

Platzman, P. M., E. D. Isaacs, H. Williams, P. Zschack, and G. E. Ice, 1992, Phys. Rev. B 46, 12943.

Plummer, W., 1997, Science 277, 1447.

Pollehn, T. J., A. Schindlmayr, and R. W. Godby, 1998, J. Phys.: Condens. Matter 10, 1273.

Porter, A. R., O. K. Al-Mushadani, M. D. Towler, and R. J. Needs, 2001, J. Chem. Phys. 114, 7795.

Pulci, O., F. Bechstedt, G. Onida, R. Del Sole, and L. Reining, 1999, Phys. Rev. B 60, 16758.

Pulci, O., G. Onida, R. Del Sole, and L. Reining, 1998, Phys. Rev. Lett. 81, 5374.

Quinn, J. J., 1962, Phys. Rev. 126, 1453.

Quinn, J. J., 1963, Appl. Phys. Lett. 2, 167.

Quinn, J. J., and R. A. Ferrell, 1958, Phys. Rev. 112, 812.

Quong, A. A., and A. G. Eguiluz, 1993, Phys. Rev. Lett. 70, 3955.

Raghavachari, K., D. Ricci, and G. Pacchioni, 2002, J. Chem. Phys. 116, 825 .

Read, A. J., and R. J. Needs, 1991, Phys. Rev. B 44, 13071.

Reining, L., and R. Del Sole, 1991, Phys. Rev. Lett. 67, 3816.

Reining, L., V. Olevano, A. Rubio, and G. Onida, 2002, Phys. Lett. 88, 066404.

Reining, L., G. Onida, and R. W. Godby, 1997, Phys. Rev. B 56, R4301.

Reining, L., O. Pulci, M. Palummo, and G. Onida, 2000, Int. J. Quantum Chem. 77, 951.

Resca, L., R. Resta, and S. Rodriguez, 1978, Phys. Rev. B 18, 696.

Resta, R., 1996, Phys. Rev. Lett. 77, 2265.

Rice, T. M., 1965, Ann. Phys. (N.Y.) 31, 100.

Richardson, C. F., and N. W. Ashcroft, 1994, Phys. Rev. B 50, 8170 .

Rignanese, G.-M., X. Blase, and S. G. Louie, 2001, Phys. Rev. Lett. 86, 2110.

Ritchie, R. H., 1959, Phys. Rev. 114, 644.

Ritchie, R. H., and J. C. Ashley, 1965, J. Phys. Chem. Solids 26, 1689.

Roberts, R. A., and W. C. Walker, 1967, Phys. Rev. 161, 730.

Roessler, D. M., and W. C. Walker, 1967, J. Opt. Soc. Am. 57, 835.

Rohlfing, M., P. Krüger, and J. Pollmann, 1995, Phys. Rev. Lett. 75, 3489.

Rohlfing, M., P. Krüger, and J. Pollmann, 1998, Phys. Rev. B 57, 6485.

Rohlfing, M., and S. G. Louie, 1998a, Phys. Rev. Lett. 80, 3320. Rohlfing, M., and S. G. Louie, 1998b, Phys. Rev. Lett. 81, 2312. Rohlfing, M., and S. G. Louie, 1999a, Phys. Rev. Lett. 82, 1959. Rohlfing, M., and S. G. Louie, 1999b, Phys. Rev. Lett. 83, 856. Rohlfing, M., and S. G. Louie, 2000, Phys. Rev. B 62, 4927.
Rohlfing, M., M. Palummo, G. Onida, and R. Del Sole, 2000, Phys. Rev. Lett. 85, 5440.

Rojas, H. N., R. W. Godby, and R. J. Needs, 1995, Phys. Rev. Lett. 74, 1827.

Rubio, A., J. A. Alonso, X. Blase, L. C. Balbás, and S. G. Louie, 1996, Phys. Rev. Lett. 77, 247; 77, E5442.

Rubio, A., J. A. Alonso, X. Blase, and S. G. Louie, 1997, Int. J. Mod. Phys. B 11, 2727.

Rubio, A., and Ll. Serra, 1993, Z. Phys. D: At., Mol. Clusters 26, S111.

Ruini, A., M. J. Caldas, G. Bussi, and E. Molinari, 2002, Phys. Rev. Lett. 88, 206403.

Runge, E., and E. K. U. Gross, 1984, Phys. Rev. Lett. 52, 997. Saile, V., 1976, Ph.D. thesis (Universität München).

Saile, V., M. Skibowski, W. Steinmann, P. Gürtler, E. E. Koch, and A. Kozevnikov, 1976, Phys. Rev. Lett. 37, 305.

Saito, S., S. B. Zhang, S. G. Louie, and M. L. Cohen, 1989, Phys. Rev. B 40, 3643.

Sánchez-Friera, P., and R. W. Godby, 2000, Phys. Rev. Lett. 85, 5611.

Schindlmayr, A., and R. W. Godby, 1998, Phys. Rev. Lett. 80, 1702.

Schindlmayr, A., T. J. Pollehn, and R. W. Godby, 1998, Phys. Rev. B 58, 12684.

Schöne, W.-D., and A. G. Eguiluz, 1998, Phys. Rev. Lett. 81, 1662.

Schöne, W.-D., R. Keyling, M. Bandić, and W. Ekardt, 1999, Phys. Rev. B 60, 8616.

Seminario, J. M., 1996, Ed., Recent Developments and Applications of Modern Density Functional Theory (Elsevier, Amsterdam).

Serra, Ll., and A. Rubio, 1997, Phys. Rev. Lett. 78, 1428.

Sham, L. J., and T. M. Rice, 1966, Phys. Rev. 144, 708.

Shirley, E. L., 1996, Phys. Rev. B 54, 7758.

Shirley, E. L., 2001, private communication.

Shirley, E. L., and R. Martin, 1993, Phys. Rev. B 47, 15404.

Silkin, V. M., T. Balasubramanian, E. V. Chulkov, A. Rubio, and P. M. Echenique, 2001, Phys. Rev. B 64, 085334.

Sinha, S. P., 1949, Proc. Phys. Soc. London 62, 124.

Smith, N., 2001, Phys. Today 54 (1), 29.

Soininen, J. A., and E. L. Shirley, 2000, Phys. Rev. B 61, 16423. Spataru, C. D., M. A. Cazalilla, A. Rubio, L. X. Benedict, P. M. Echenique, and S. G. Louie, 2001, Phys. Rev. Lett. 87, 246405.

Stener, M., P. Decleva, and A. Görling, 2001, J. Chem. Phys. 114, 7816.

Stiebling, J., 1978, Z. Phys. B 31, 355.

Stott, M. J., and E. Zaremba, 1980, Phys. Rev. A 21, 12.

Strinati, G., 1982, Phys. Rev. Lett. 49, 1519.

Strinati, G., 1984, Phys. Rev. B 29, 5718.

Strinati, G., 1988, Riv. Nuovo Cimento 11, 1.

Strinati, G., H. J. Mattausch, and W. Hanke, 1980, Phys. Rev. Lett. 45, 290.

Strinati, G., H. J. Mattausch, and W. Hanke, 1982, Phys. Rev. B 25, 2867.

Strocov, V. N., R. Claessen, G. Nicolay, S. Hüfner, A. Kimura, A. Harasawa, S. Shin, A. Kakizaki, P. O. Nilsson, H. I. Starnberg, and P. Blaha, 1998, Phys. Rev. Lett. 81, 4943.

Strocov, V. N., R. Claessen, G. Nicolay, S. Hüfner, A. Kimura, A. Harasawa, S. Shin, A. Kakizaki, H. I. Starnberg, P. O. Nilsson, and P. Blaha, 2001, Phys. Rev. B 63, 205108.

Sturm, K., 1995, Phys. Rev. B 52, 8028.

Sturm, K., and A. Gusarov, 2000, Phys. Rev. B 62, 16474. 
Sundström, V. S., 1996, Ed., Femtosecond Chemistry and Femtobiology: Ultrafast Reaction Dynamics of Atomic Scale Resolution, Nobel Symposium 101 (Imperial College Press, London).

Szabo, A., and N. S. Ostlund, 1983, Modern Quantum Chemistry (MacMillan, New York).

Tatarczyk, K., A. Schindlmayer, and M. Scheffler, 2001, Phys. Rev. B 63, 235106.

Taut, M., 1992, J. Phys. C 4, 9595.

Tokatly, I. V., and O. Pankratov, 2001, Phys. Rev. Lett. 86, 2078.

Tsuei, K. D., E. W. Plummer, A. Liebsch, K. Kempa, and P. Bakshi, 1990, Phys. Rev. Lett. 64, 44.

Ummels, R. T. M., P. A. Bobbert, and W. van Haeringen, 1998, Phys. Rev. B 57, 11962.

Valla, T., A. V. Fedorov, P. D. Johnson, B. O. Wells, S. L. Hulbert, Q. Li, G. D. Gu, and N. Koshizuka, 1999a, Science 285, 2110.

Valla, T., A. V. Fedorov, P. D. Johnson, and S. L. Hulbert, 1999b, Phys. Rev. Lett. 83, 2085.

van der Horst, J.-W., P. A. Bobbert, M. A. J. Michels, G. Brocks, and P. J. Kelly, 1999, Phys. Rev. Lett. 83, 4413.

van Dyke, J. P., 1972, Phys. Rev. B 5, 1489.

van Gelderen, P., P. A. Bobbert, P. J. Kelly, and G. Brocks, 2000, Phys. Rev. Lett. 85, 2989.

van Leeuwen, R., 1998, Phys. Rev. Lett. 80, 1280.

van Leeuwen, R., 1999, Phys. Rev. Lett. 82, 3863.

van Leeuwen, R., 2001, Int. J. Mod. Phys. B 15, 1969.

van Leeuwen, R., and E. J. Baerends, 1994, Phys. Rev. A 49, 2421.

Vasiliev, I., S. Ögüt, and J. R. Chelikowsky, 1999, Phys. Rev. Lett. 82, 1919.

Vasiliev, I., S. Öğüt, and J. R. Chelikowsky, 2002, Phys. Rev. B 65, 115416.

Vast, N., L. Reining, V. Olevano, P. Schattschneider, and B. Jouffrey, 2002, Phys. Rev. Lett. 88, 037601.
Verdozzi, C., M. Cini, and A. Marini, 2001, J. Electron Spectrosc. Relat. Phenom. 117-118, 41.

Verdozzi, C., R. W. Godby, and S. Holloway, 1995, Phys. Rev. Lett. 74, 2327.

Vignale, G., 1995, Phys. Rev. Lett. 74, 3233.

Vignale, G., and W. Kohn, 1996, Phys. Rev. Lett. 77, 2037.

Vignale, G., C. A. Ullrich, and S. Conti, 1997, Phys. Rev. Lett. 79, 4878.

von Barth, U., and B. Holm, 1996, Phys. Rev. B 54, 8411.

von der Linden, W., and P. Horsch, 1988, Phys. Rev. B 37, 8351.

Vos, M., A. S. Kheifets, E. Weigold, and F. Aryasetiawan, 2001, Phys. Rev. B 63, 033108.

Vukajlovic, F. R., E. L. Shirley, and R. M. Martin, 1991, Phys. Rev. B 43, 3994.

Waidmann, S., M. Knupfer, B. Arnold, J. Fink, A. Fleszar, and W. Hanke, 2000, Phys. Rev. B 61, 10149.

Wang, C. R. C., S. Pollack, D. Cameron, and M. M. Kappes, 1990, J. Chem. Phys. 93, 3787.

Wannier, G. H., 1937, Phys. Rev. 52, 191.

White, I. D., R. W. Godby, M. M. Rieger, and R. J. Needs, 1998, Phys. Rev. Lett. 80, 4265.

Wigner, E. P., 1934, Phys. Rev. 46, 1002.

Wiser, N., 1963, Phys. Rev. 129, 62.

Yabana, K., and G. F. Bertsch, 1996, Phys. Rev. B 54, 4484.

Yabana, K., and G. F. Bertsch, 1999a, Int. J. Quantum Chem. 75, 55.

Yabana, K., and G. F. Bertsch, 1999b, Phys. Rev. A 60, 1271.

Yasuhara, H., S. Yoshinaga, and M. Higuchi, 1999, Phys. Rev. Lett. 83, 3250.

Yasuhara, H., S. Yoshinaga, and M. Higuchi, 2000, Phys. Rev. Lett. 85, 2411.

Zangwill, A., and P. Soven, 1980, Phys. Rev. A 21, 1561.

Zhou, J., P. Bogdanov, S. A. Kellar, T. Noda, H. Eisaki, S. Uchida, Z. Hussain, and Z.-X. Shen, 1999, Science 286, 268.

Zimmermann, R., 1970, Phys. Status Solidi 41, 23. 